\title{
Small Modular Reactor: First-of-a-Kind (FOAK) and Nth-of-a-Kind (NOAK) Economic Analysis
}

\section{Idaho National Laboratory Summer 2014 Report}

Lauren M. Boldon and Piyush Sabharwall

\author{
August 2014
}

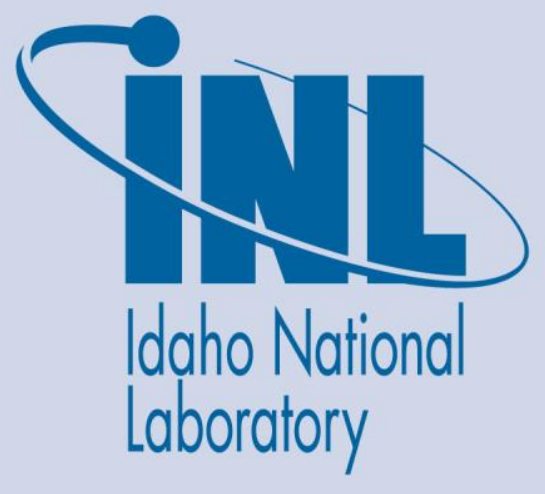

The INL is a U.S. Department of Energy National Laboratory operated by Battelle Energy Alliance 


\section{DISCLAIMER}

This information was prepared as an account of work sponsored by an agency of the U.S. Government. Neither the U.S. Government nor any agency thereof, nor any of their employees, makes any warranty, expressed or implied, or assumes any legal liability or responsibility for the accuracy, completeness, or usefulness, of any information, apparatus, product, or process disclosed, or represents that its use would not infringe privately owned rights. References herein to any specific commercial product, process, or service by trade name, trade mark, manufacturer, or otherwise, does not necessarily constitute or imply its endorsement, recommendation, or favoring by the U.S. Government or any agency thereof. The views and opinions of authors expressed herein do not necessarily state or reflect those of the U.S. Government or any agency thereof. 
INL/EXT-14-32616

Revision 0

\section{Small Modular Reactor: \\ First-of-a-Kind (FOAK) and Nth-of-a-Kind (NOAK) Economic Analysis}

INL Summer 2014 Report

Lauren M. Boldon and Piyush Sabharwall

August 2014

Idaho National Laboratory

Nuclear Energy University Programs

Idaho Falls, Idaho 83415

http://www.inl.gov

Prepared for the

U.S. Department of Energy

Office of Nuclear Energy

Under DOE Idaho Operations Office

Contract DE-AC07-05ID14517 


\section{ABSTRACT}

Small modular reactors (SMRs) refer to any reactor design in which the electricity generated is less than $300 \mathrm{MWe}$. Often medium-sized reactors with power less than $700 \mathrm{MWe}$ are also grouped into this category. Internationally, the development of a variety of designs for SMRs is booming with many designs approaching maturity and even in or nearing the licensing stage. It is for this reason that a generalized yet comprehensive economic model for first-of-a-kind (FOAK) through nth-of-a-kind (NOAK) SMRs based upon rated power, plant configuration, and the fiscal environment was developed. In the model, a particular project's feasibility is assessed with regards to market conditions and by commonly utilized capital budgeting techniques, such as the net present value (NPV), internal rate of return (IRR), Payback, and more importantly, the levelized cost of energy (LCOE) for comparison to other energy production technologies. Finally, a sensitivity analysis was performed to determine the effects of changing debt, equity, interest rate, and conditions on the LCOE. The economic model is primarily applied to the near future water-cooled SMR designs in the United States. Other gas-cooled and liquid metal-cooled SMR designs have been briefly outlined in terms of how the economic model would change.

FOAK and NOAK SMR costs were determined for a site containing seven 180-MWe water-cooled SMRs and compared to a site containing one 1260-MWe reactor. With an equal share of debt and equity and a $10 \%$ cost of debt and equity, the LCOE was determined to be $\$ 67-\$ 84 / \mathrm{MWh}$ and $\$ 89 / \mathrm{MWh}$ for the SMR and large reactor sites, respectively, demonstrating how SMRs have a higher rate of return and lower operational costs that typical large commercial reactors.

To assess the feasibility of SMRs in more realistic scenarios, such as part of hybrid energy systems or combined heat and power facilities, three cases were performed including electric-only base, storage, and hydrogen production cases; all these cases were used for a preliminary economic analysis of a SMR-wind-natural gas hybrid energy system for the New York West Central region. A nearly $4 \%$ increase in profits was observed for the storage case over the electric-only base case, demonstrating how pairing nuclear technology with devices which improve load following capabilities will help support future nuclear energy development. 


\section{ACKNOWLEDGMENTS}

I would like to thank Daniel Curtis, Massachusetts Institute of Technology, and Erich Schneider, University of Texas at Austin, for their contributions and continued support of this work. I would also like to thank the DOE for its support through the Nuclear Energy University Program Graduate Fellowship. Any opinions, findings, conclusions or recommendations expressed are those of the author(s) and do not necessarily reflect the view of the Department of Energy Office of Nuclear Energy.

\section{CONTENTS}

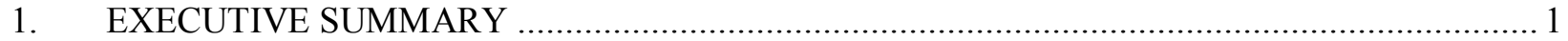

2. SMR DEVELOPMENT IN THE UNITED STATES ...................................................... 2

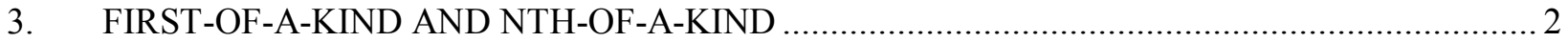

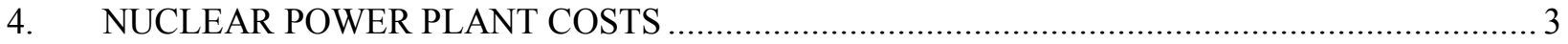

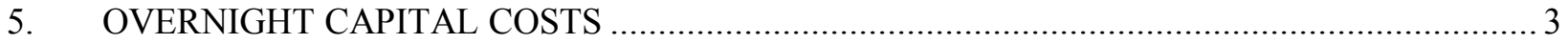

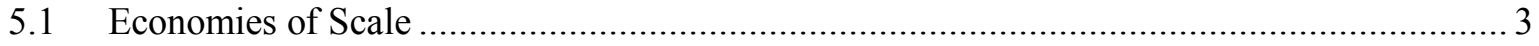

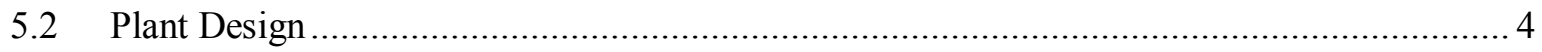

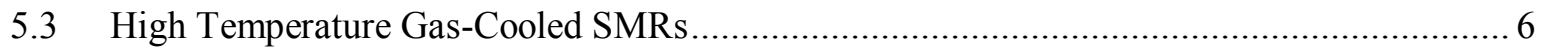

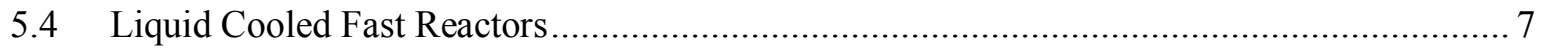

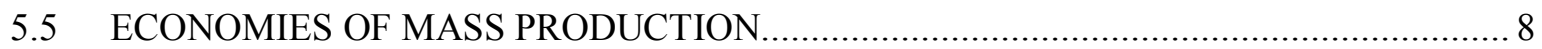

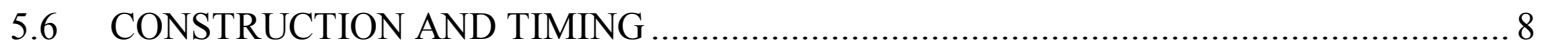

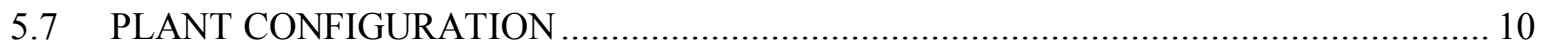

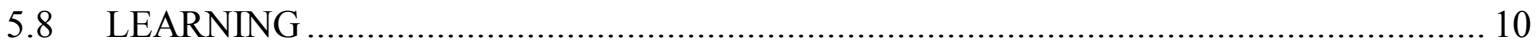

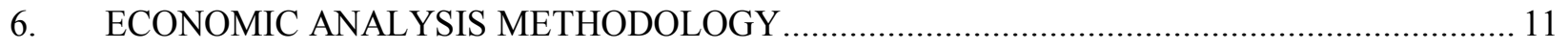

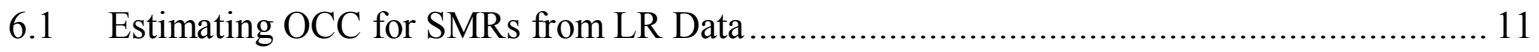

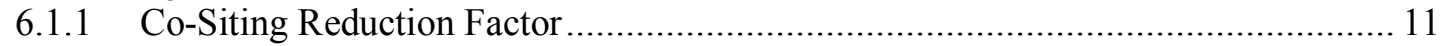

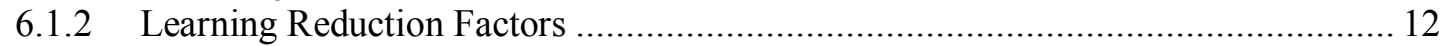

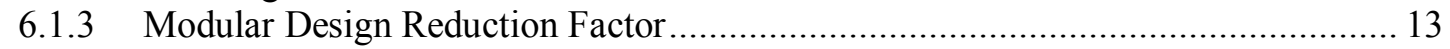

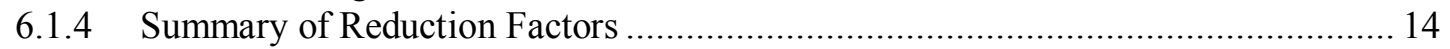

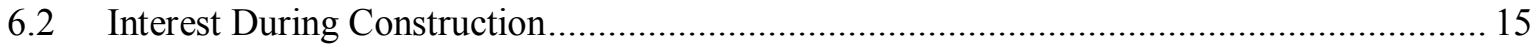

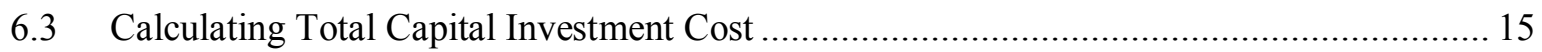

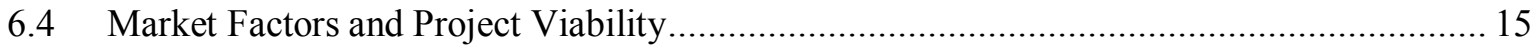

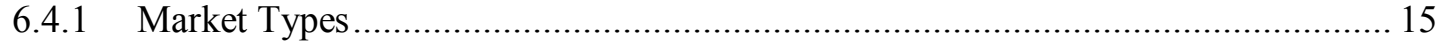

6.4.2 Weighted Average Cost of Capital ................................................................... 16

6.4.3 Real and Nominal Discount Rate Methods ...................................................... 16

6.4.4 Fixed Charge Rate and Amortization of Capital Costs ........................................... 16

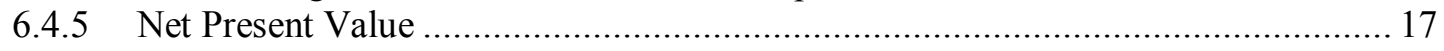

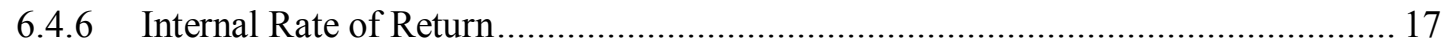

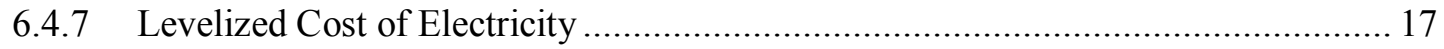




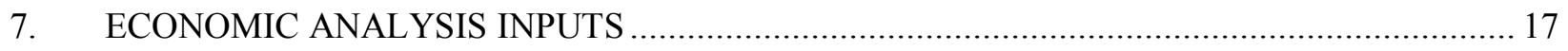

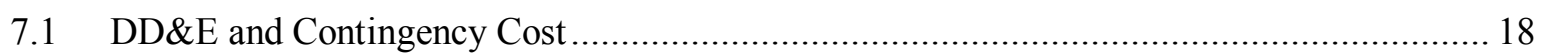

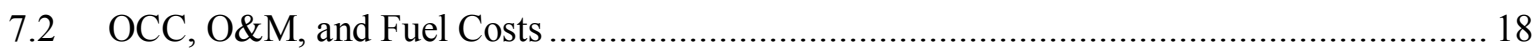

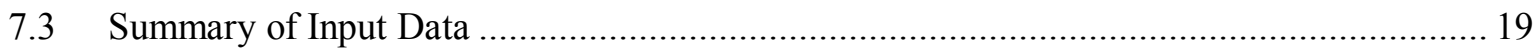

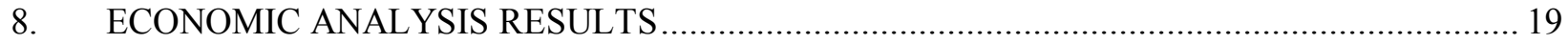

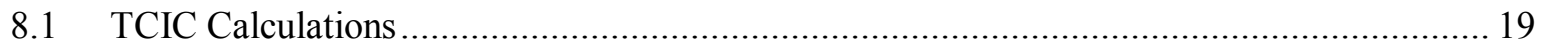

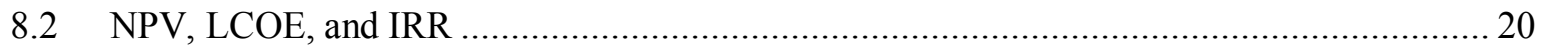

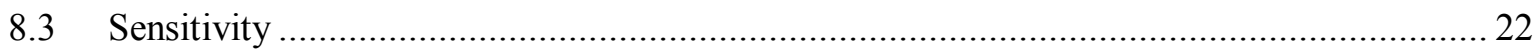

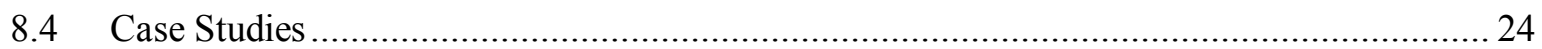

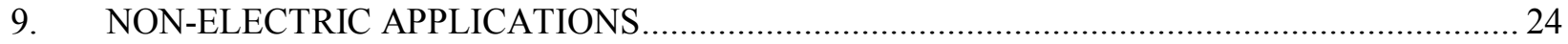

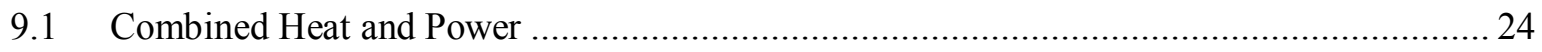

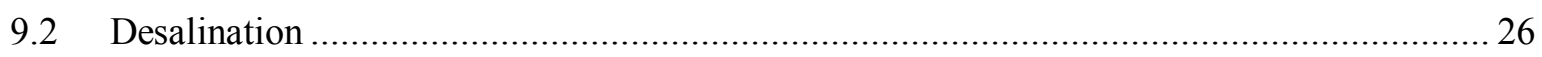

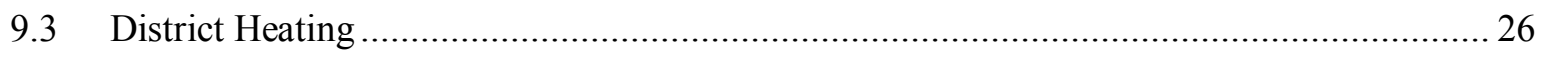

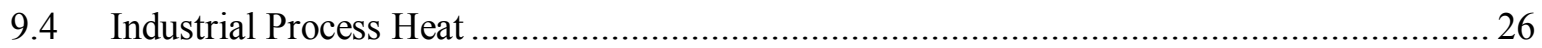

10. NUCLEAR HYBRID ENERGY SYSTEMS (NHES) UTILIZING SMRS................................ 27

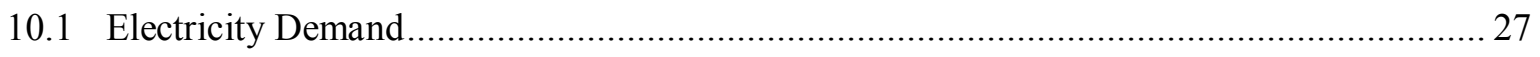

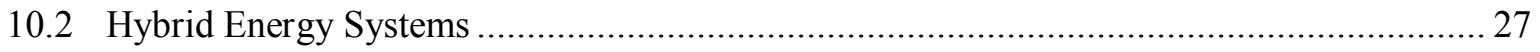

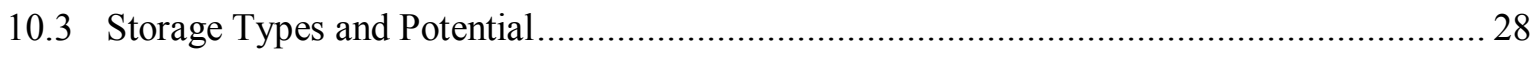

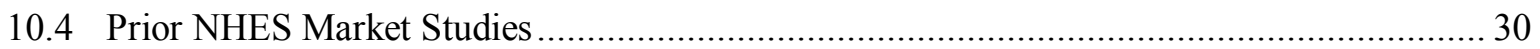

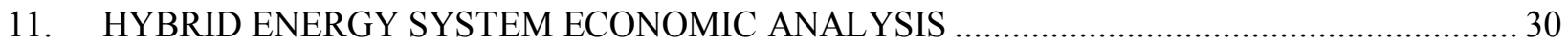

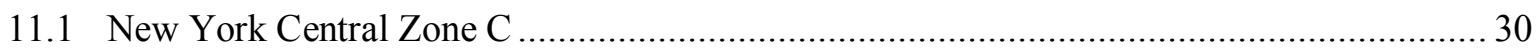

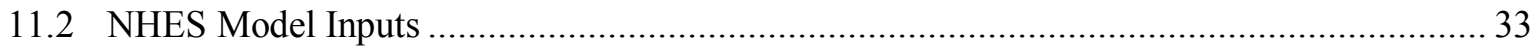

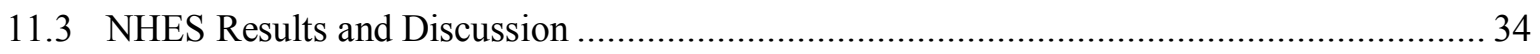

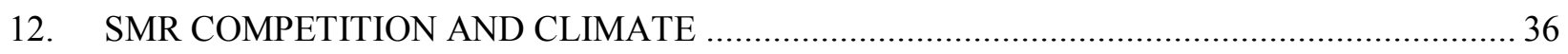

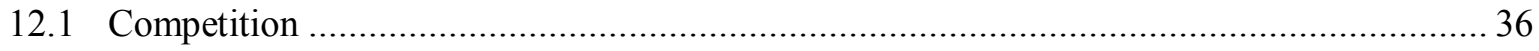

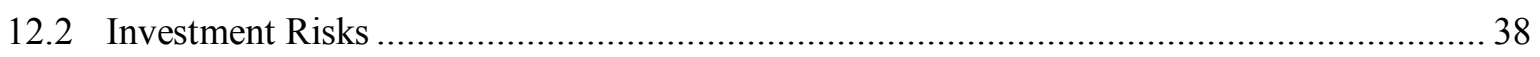

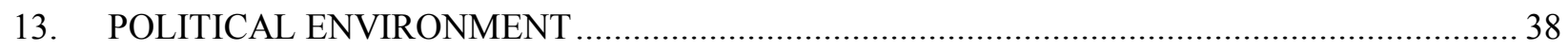

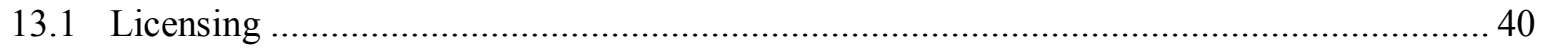

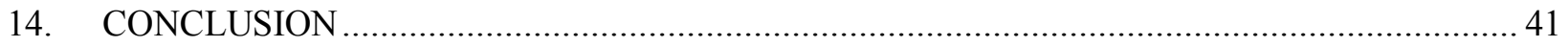

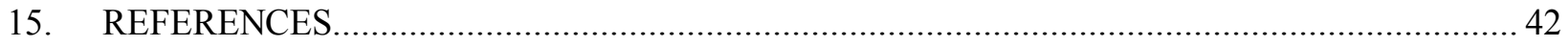

Appendix A NPP Detailed Cost Breakdown (GIF Code of Accounts) …............................................ 46

Appendix B Real and Nominal Discount Rate Methods .................................................................. 48

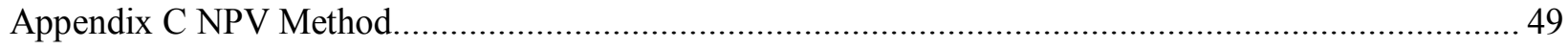

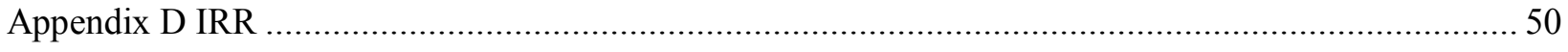




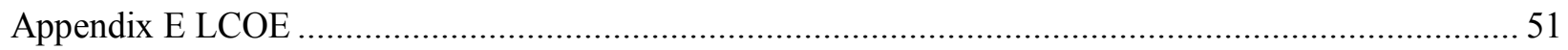

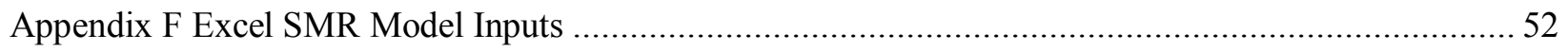

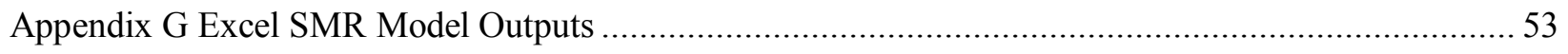

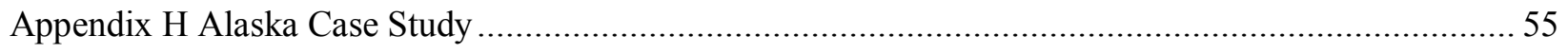

Appendix I Electricity Markets, Locational Based Marginal Pricing and Merit Order Usage ................. 57

Appendix J Excel NHES Model Inputs and General Methodology ................................................ 58

\section{FIGURES}

Figure 1. Example economies of scale and reduction factors for 180 MWe SMR. ............................... 3

Figure 2. Example economies of scale and reduction factors for 180 MWe SMR.Error! Bookmark not defined.

Figure 3. HTGR past reactors (PNNL 2011) ....................................................................... 6

Figure 4. Cogeneration process temperatures (INL NGNP) ................................................. 7

Figure 5. Serial production effects for nuclear propulsion reactors (Mitenkov et al. 2004)..................... 8

Figure 6. United States large reactor construction times in months (Schneider, Frogatt, and

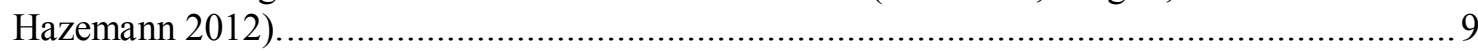

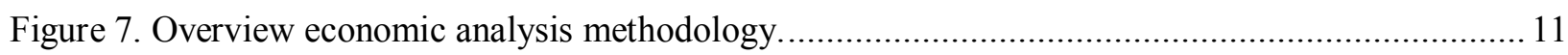

Figure 8. Effects of modularity as a function of rated power (MWe) (Reid 2003) ............................... 14

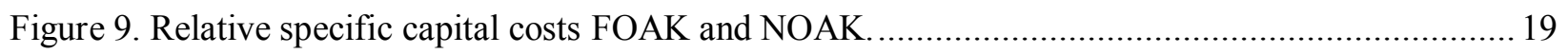

Figure 10. Cumulative cash flows for a seven-unit 180-MWe SMR plant with the deployment

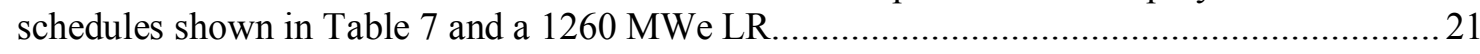

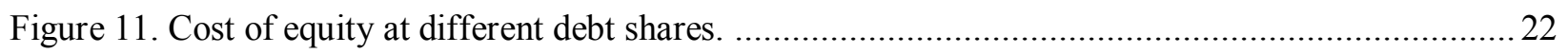

Figure 12. LCOE sensitivity to debt and equity shares, cost of equity, and cost of debt.........................23

Figure 13. NPV sensitivity to debt and equity shares, cost of equity, and cost of debt.........................2 23

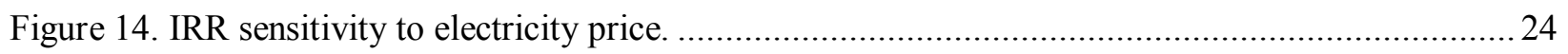

Figure 15. Example CHP facility (EPA Combined Heat and Power Partnership 2013). ...................... 25

Figure 16. Example CHP Facility (EPA Combined Heat and Power Partnership 2013). ...................... 26

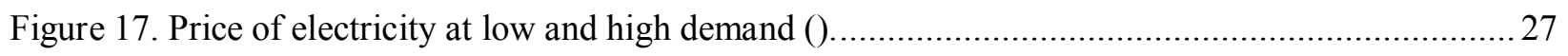

Figure 18. Illustration of a possible nuclear hybrid energy storage system (Ruth et al. 2014)................28

Figure 19. Types of HES and relative discharge times and power ratings (Akhhil et al. 2013). ............. 29

Figure 20. Daily power demand pattern based on season (NY Affordable Reliable Electricity

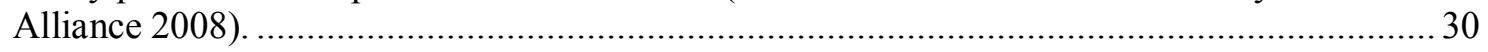

Figure 21. Correlation between natural gas and electricity prices in New England (Anjum 2013).......... 31

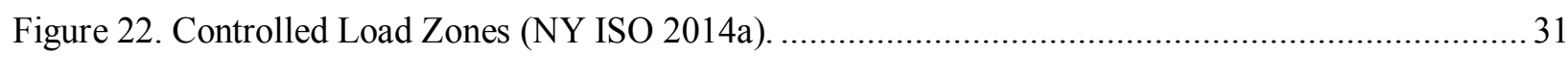


Figure 23. Typical Summer Load in Region C (6/25-6/26): dashed is predicted, regular line is actual (NY ISO 2014b).

Figure 24. Typical Marginal Cost of Electricity in Region C (6/25-6/26) (NY ISO 2014b)

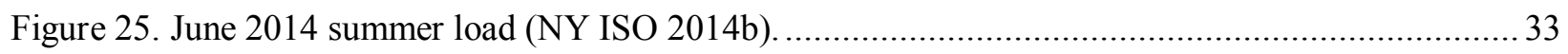

Figure 26. Profit and cost shares for Base Case and Storage Case with CAES. ................................... 35

Figure 27. Profit and revenue shares for Hydrogen Production Case. ............................................. 36

Figure 28. External cost comparison in Germany, including global warming costs (IAEA 2007)............ 39

Figure 29. Total electricity generating costs for different technologies in Germany, including external costs (IAEA 2007).

\section{TABLES}

Table 1. U.S. SMR designs in the design and/or licensing stages (NEA and OECD 2011)....................2

Table 2. Pressurized water SMR design features and their targeted safety improvements and

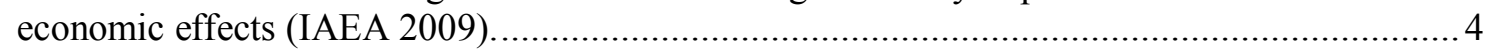

Table 3. Sample learning rates and level of maturity (NETL 2013) ................................................. 13

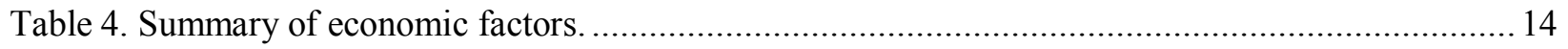

Table 5. Summary of SMR data required for economic model. ......................................................... 19

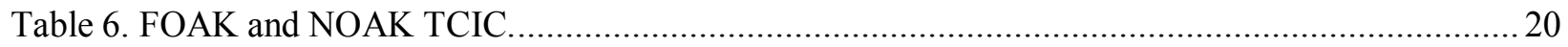

Table 7. Example SMR construction deployment schedules......................................................... 20

Table 8. NPV, IRR, required electricity price, payback, and LCOE for seven-unit 180-MWe

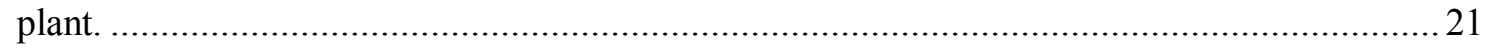

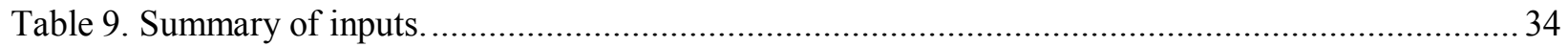

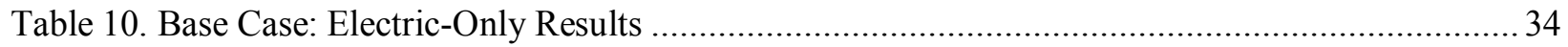




\section{ACRONYMS}

\begin{tabular}{|c|c|}
\hline ABWR & Advanced Boiling Water Reactor \\
\hline CAES & Compressed Air Energy Storage \\
\hline $\mathrm{CDF}$ & Core Damage Frequency \\
\hline CHP & Combined Heat and Power \\
\hline DD\&E & Detail Design and Engineering \\
\hline EMWG & Economic Modeling Working Group \\
\hline FCR & Fixed Charge Rate \\
\hline FERC & Federal Energy Regulatory Commission \\
\hline FOAK & First-of-a-Kind \\
\hline GHG & Greenhouse Gas \\
\hline GWe & Gigawatt \\
\hline HTG & High Temperature Gas-Cooled \\
\hline HTGR & High Temperature Gas-Cooled Reactor \\
\hline HTR & High Temperature Reactor \\
\hline HTTR & High Temperature Engineering Test Reactor \\
\hline IDC & Interest During Construction \\
\hline IRR & Internal Rate of Return \\
\hline ISC & Independent System Operator \\
\hline LCOE & Levelized Cost of Energy \\
\hline LERF & Large Early Release Frequency \\
\hline LOCA & Loss of Coolant Accident \\
\hline LOFA & Loss of Flow Accident \\
\hline LR & Large Reactor \\
\hline LWR & Light Water Reactor \\
\hline MWe & Megawatt electric \\
\hline MWth & Megatwatt thermal \\
\hline NGNP & Next Generation Nuclear Plant \\
\hline NHES & Nuclear Hybrid Energy System \\
\hline NOAK & Nth-of-a-Kind \\
\hline NPP & Nuclear Power Plant \\
\hline NPV & Net Present Value \\
\hline NRC & Nuclear Regulatory Commission \\
\hline O\&M & Operation and Maintenance \\
\hline
\end{tabular}




$\begin{array}{ll}\text { OCC } & \text { Overnight Capital Cost } \\ \text { PM } & \text { Pebble-bed Module } \\ \text { PWR } & \text { Pressurized Water Reactor } \\ \text { SMR } & \text { Small Modular Reactor } \\ \text { TCIC } & \text { Total Capital Investment Cost } \\ \text { TRISO } & \text { Tristructural Isotropic } \\ \text { WACC } & \text { Weighted Average Cost of Capital }\end{array}$




\section{Small Modular Reactor: First-of-a-Kind (FOAK) and Nth-of-a-Kind (NOAK) Economic Analysis}

\section{EXECUTIVE SUMMARY}

Nuclear energy is a clean air base-load energy production method that accounts for $19 \%$ of total electricity generation and 70\% of clean air generation in the United States (NEI 2014). With 100 reactors currently operating and demonstrating high availability, safety, and reliability, nuclear energy is a potential solution to reduce carbon emissions and the United States' dependence on fluctuating fossil fuel prices (NEI 2014). However, 1+ GW large reactors (LR) require substantial upfront investments, making the financing of new nuclear projects extremely difficult due to financial, regulatory, political, and technological risks. Small Modular Reactor (SMR) development, on the other hand, requires substantially less upfront capital costs, resulting in reduced financial risks and providing an attractive alternative to large reactors.

SMRs may be used to compete on a regular electricity market or in niche applications (NEA and OECD 2011). The flexible deployment and operation schedules coupled with faster onsite modular assembly endear SMRs to a variety of settings, which would be less feasible for large reactors or other generation technologies. For example, small remote villages in Alaska pay extremely high electricity prices, as do island populations in Hawaii. These groups would benefit greatly from SMRs with reduced electricity prices, generated by clean air technology and a reduction in fossil fuel dependence.

Additionally, SMRs may provide energy in locations striving to add renewable energies or where the common renewable energies, such as wind and solar, are not feasible. Hawaii, for example, has limited land availability, and wind and solar energy, although feasible, would require significant land use.

SMRs may also be fitted to specific cogeneration applications, such as providing electricity and/or heat for desalination, district heating, industrial process heat, and even hydrogen production (IAEA 2007). They may also be combined with Hybrid Energy Storage systems to manage fluctuations in intermittent renewable energy generation while also storing energy or providing electricity/heat for cogeneration. SMRs may be phased in slowly to replace aging plants, such as coal plants that would be difficult to retrofit with newer carbon capture and storage technologies (Rosner and Goldberg 2011). Additionally, the number of SMRs on a site and the rated power for each is flexible, as designers aim to develop them for a wide array of applications.

The specific capital costs for SMRs tend to be significantly higher than those of a large reactor, as the costs are distributed over drastically reduced production levels. However, the specific capital may be reduced through the use of economies of mass production, multiples, and modularity, and configurational and technological learning (NEA and OECD 2011, Rosner and Goldberg 2011). To reach a point in which enough SMRs, or nth-of-a-kind (NOAK) reactors, are being manufactured to warrant these cost reductions and for the design and licensing costs to be mitigated, significant investment would be required (Rosner and Goldberg 2011).

The purpose of this study is to develop a general methodology of assessing the economics of First-of-a-Kind (FOAK) and Nth-of-a-Kind (NOAK) Small Modular Reactors and SMR Hybrid Energy Systems in order to determine the conditions which would make them competitive with other generation technologies. This report provides background on the factors that affect the nuclear reactor capital and operational costs; details technology independent cost estimates for water-cooled SMRs; develops scenarios which would support future SMR development, including the operational revenue and cost data for an SMR-wind-natural gas hybrid energy system with storage and/or hydrogen sales; and provides a qualitative review of licensing and economic challenges for future SMR development. 


\section{SMR DEVELOPMENT IN THE UNITED STATES}

In the United States, very few utility companies have the required equity to finance the large upfront capital costs associated with reactors over $700 \mathrm{MWe}$ (Locatelli, Bingham, and Mancini 2014). Therefore, many new SMR designs are being seriously considered and are in the design, research and development, or licensing stages. Table 1 displays the SMR designs, which have nearly reached design completion, along with their expected deployment dates (NEA and OECD 2011). Because the United States has extensive historical experience with light water reactors (LWRs), all of the new SMR designs are also water-cooled reactors, making it much more likely that the Nuclear Regulatory Commission (NRC) will issue construction and operating licenses. Newer designs, such as Liquid Metal Reactors are a more difficult sell, as there is little historical operating experience to demonstrate operational safety and the ability to respond effectively during an accident.

Table 1. U.S. SMR designs in the design and/or licensing stages (NEA and OECD 2011).

\begin{tabular}{|l|c|l|l|l|}
\hline \multicolumn{1}{|c|}{ SMR } & $\begin{array}{c}\text { Type } \\
\text { of } \\
\text { Reactor }\end{array}$ & $\begin{array}{c}\text { Rated } \\
\text { Power }\end{array}$ & \multicolumn{1}{|c|}{ Stage in Design/Licensing } & \multicolumn{1}{c|}{$\begin{array}{c}\text { Expected } \\
\text { Deployment }\end{array}$} \\
\hline NuScale & PWR & $45 \mathrm{MWe}$ & $\begin{array}{l}\text { NRC Pre-application Review Phase, } \\
\text { Design Certification Application to be } \\
\text { submitted in 2015 (NuScale Power 2014). }\end{array}$ & $\begin{array}{l}\text { FOAK 2018 } \\
\text { (NEA and OECD } \\
\text { 2011) }\end{array}$ \\
\hline mPower & PWR & $180 \mathrm{MWe}$ & $\begin{array}{l}\text { NRC Pre-application, Design } \\
\text { Certification Application and } \\
\text { Construction Permit Application to be } \\
\text { submitted 2013 (NRC 2014). }\end{array}$ & $\begin{array}{l}\text { 2018 (NEA and } \\
\text { OECD 2011) }\end{array}$ \\
\hline IRIS & PWR & $335 \mathrm{MWe}$ & $\begin{array}{l}\text { NRC Pre-application Review Phase, } \\
\text { expected Final Design Approval in 2012 } \\
\text { (Kling and Carelli 2006). }\end{array}$ & $\begin{array}{l}\text { 2015-2017 } \\
\text { (Kling and } \\
\text { Carelli 2006) }\end{array}$ \\
\hline $\begin{array}{l}\text { Westinghouse } \\
\text { SMR }\end{array}$ & PWR & $225+$ MWe & \multicolumn{1}{c}{ - } & \\
\hline
\end{tabular}

\section{FIRST-OF-A-KIND AND NTH-OF-A-KIND}

The Economic Modeling Working Group (EMWG) defines the first-of-a-kind (FOAK) plant as the "first commercial plant built with estimated equipment, materials, and labor productivity based on current or recent nuclear plant experience" (Generation IV EMWG 2006). It refers to the first commercial plant of a particular design where prior operational experiences and lessons learned will play a part, but ultimately the majority of experience is to be gained during operation of this specific plant design. It is for this reason that FOAK plants are traditionally $15-55 \%$ more expensive than subsequent non-FOAK plants (NEA and OECD 2011). The one-time costs associated with a FOAK facility, such as design and licensing costs may be leveraged over all the plants from FOAK up to but not including the NOAK plant per the EMWG guidelines (Generation IV EMWG 2006).

The NOAK plant is defined by EMWG as an "identical plant supplied and built by (the) same vendors and contractors as the FOAK plant with only the site specific scope adopted for the NOAK plant site needs. NOAK costs are achieved for the next plant after 8 gigawatts (GWe) of capacity (for large reactors) has been constructed of a particular nuclear energy system." (Generation IV EMWG 2006). Due to costs associated with fuel processing, the EMWG assumes that a nuclear fleet of at least 32 GWe will be developed (Generation IV EMWG 2006). For water-cooled SMR designs, this is unnecessary as the large LWR fleet currently operating in the U.S. is already supporting these facilities; however, once 
enough SMRs have been constructed, additional facilities may be required, thereby validating this assumption. On the other hand, for non-water-cooled designs, this assumption may prove accurate.

\section{NUCLEAR POWER PLANT COSTS}

All nuclear power plant (NPP) capital costs may be simplified into two major categories: capital and operational costs. Capital costs refer to the upfront costs associated with the plant design, licensing, and construction processes. They are often grouped into detailed design and engineering (DD\&E), overnight capital costs (OCC), interest during construction (IDC), and contingency costs. Operational costs, on the other hand, refer to the personnel, materials, and equipment required for plant operations. This may be further broken down into operations and maintenance (O\&M), fuel, and decommissioning costs. Appendix A contains a more detailed NPP cost breakdown. This report assumes that the majority of LR costs are applicable to SMRs. However, this may not be the case. Historical cost overruns for LRs were primarily due to licensing and/or construction delays. These delays would apply to SMRs, but are likely mitigated by reduced upfront costs, modular design, and faster assembly.

\section{OVERNIGHT CAPITAL COSTS}

\subsection{Economies of Scale}

When estimating OCC for a new product or service in which no exact cost data is available, it is necessary to extrapolate the necessary information from well-known products and services and alter this information to accommodate any major design differences. To scale the costs associated with a large-scale reactor to those of an SMR, one must follow an economy of scale curve based on plant capacity; this curve implies that the specific capital cost, or the cost per $\mathrm{kW}$ of electricity, will decrease with increasing plant capacity, as shown in Figure 1. On the other hand, the total capital cost will increase with increasing capacity, following an inverted economy of scale curve (Figure 2). Capital costs and in some instances labor costs will follow economies of scale (Rothwell 2007). This economic model does not separately account for the effects of scaling on labor costs.

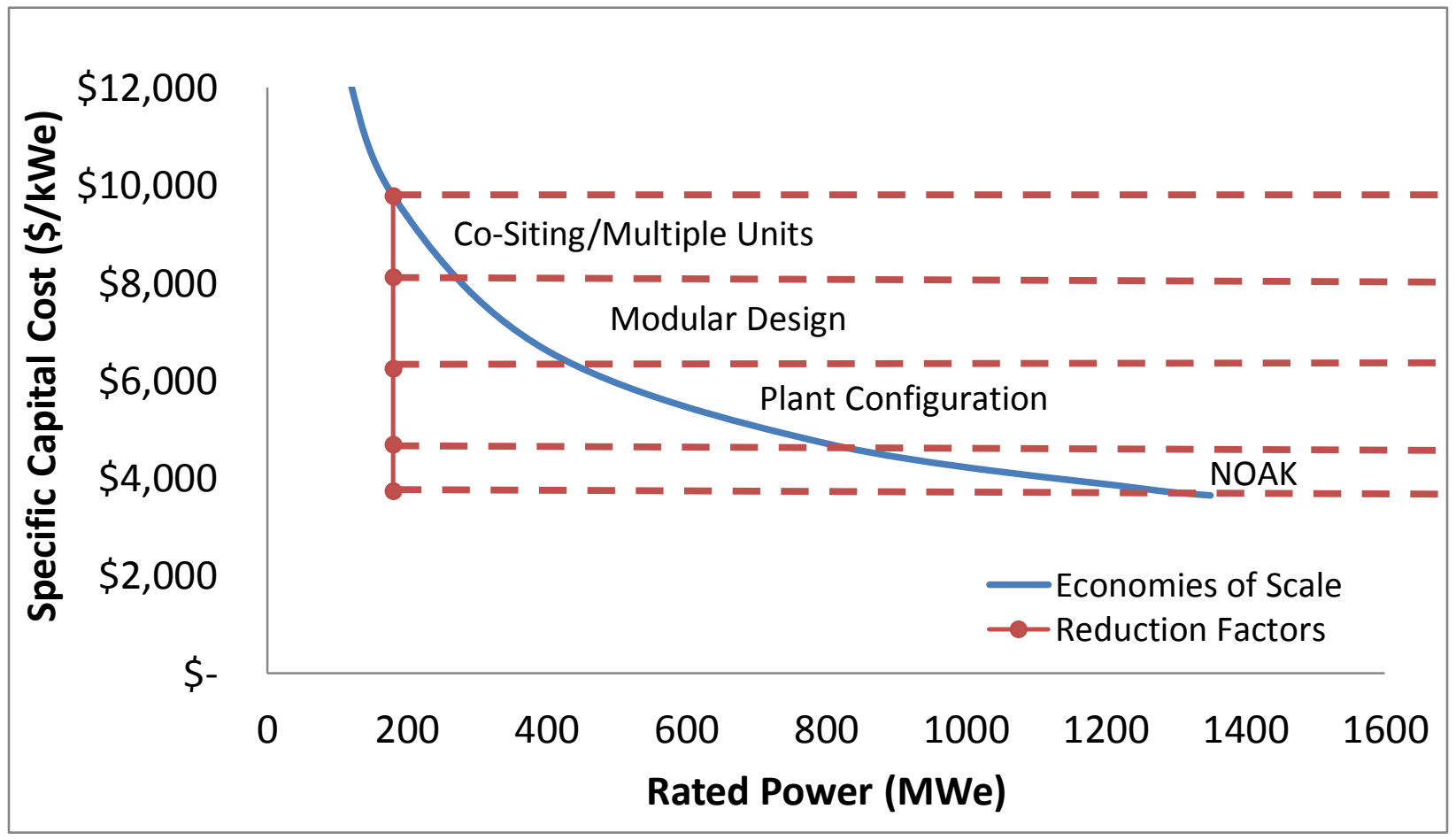

Figure 1. Example economies of scale and reduction factors for 180 MWe SMR. 
Other factors resulting from differences in SMR designs and applications may decrease the specific capital costs. The ultimate goal for an SMR project is to create an economically advantageous environment for development in which these factors make SMRs competitive with other forms of electricity generation. Several widely accepted factors that demonstrate greatly reduced specific capital required are those displayed in Figure 1 (NEA and OECD 2011; Kuznetsov 2008; Carelli, Petrovic, and Mycoff 2007; Wilton 2012).

Although there is agreement that these factors alter the required capital costs, the degree to which they do varies and is approximated utilizing several distinct methods and assumptions. Two major design factors, which will be treated separately from plant design, are the factory fabrication of modular units and learning due to plant configuration. Modularization allows for serial component fabrication and economies of mass production to take effect (Carelli, Petrovic, and Mycoff 2007; Carelli, Petrovic, Cavlina, and Grgic 2005). The plant configuration may affect the capital costs by maximizing the shared facilities, personnel, and components (Carelli, Petrovic, Cavlina, and Grgic 2005). A discussion of each of these factors and some additional considerations are presented in the following sections. Other considerations that are not further detailed in this report, but should be briefly mentioned are the ability to more closely match the supply and demand and toreduce typical grid instabilities that occur with addition of new electricity to a grid. (Carelli, Petrovic, and Mycoff 2007).

\subsection{Plant Design}

Plant designs for SMRs may incorporate advanced technologies, such as natural circulation and gravity driven systems rather than pumps; often these passive and/or advanced systems would not be feasible in large reactors (Locatelli, Bingham, and Mancini 2014, Rosner and Goldberg 2011). It is due to these inherent advanced technologies and resulting enhanced safety features that SMRs may be sited near cities or in rural areas over a wide range of output capacities and even for different cogeneration applications (Locatelli, Bingham, and Mancini 2014, IAEA 2007).

In the United States, defense-in-depth is integral in nuclear reactor design. For SMRs, this is provided through Safety-by-Design in which design simplifications and the addition of inherent, passive systems are meant to eliminate initiating events where possible and to minimize the potential consequences when they cannot be eliminated (Kuznetsov 2008; Carelli, Petrovic, Cavlina, and Grgic 2005). Active systems may also be used to mitigate the effects and to reduce the core damage frequency for higher levels of defense-in-depth (Carelli, Petrovic, Cavlina, and Grgic 2005). The simplification or reduction in the number of components and passive systems ultimately leads to improved operability of the plant, increased capacity factors, a reduction in environmental impacts due to waste, reduced construction times, and a reduction in the components that must be actively maintained (Carelli et al 2004; IAEA 2009).

Less human intervention means reduced O\&M qualifications and training and fewer actions performed which could result in human error, effectively reducing the O\&M cost and improving plant safety and performance (IAEA 2009). This also applies to accident scenarios, as the passive systems may be self-controlled with passive shutdown capabilities, limiting the human intervention required and the potential for sabotage (Rosner and Goldberg 2011; IAEA 2009). The enhanced safety provided by both advanced and passive systems may result in greater proliferation resistance, less emergency response requirements, and even a diminished emergency planning zone, further reducing O\&M costs with less personnel training and infrastructure (Carelli, Petrovic, and Mycoff 2007; Carelli, Petrovic, Cavlina, and Grgic 2005; IAEA 2009; Killich, Ramsden, and Melaina 2012; IAEA 2006).

Modular designs require more compact systems, so that factory fabrication may occur and take full advantage of economies of mass production. This is done through simplification, reduced containment, and other design features specific to the particular SMR, such as an integral design (IAEA 2009). ${ }^{\text {Error!' }}$ eference source not found. The compactness of a design has a direct role in safety, as well; for example, the integral design is extremely compact with the core, steam generators, and control rod drives all located within the pressure vessel, making sabotage or other security threats less likely (Rosner and Goldberg 
2011; Carelli, Petrovic, and Mycoff 2007). Another key design feature of many SMRs is an extended fuel cycle, which requires significantly fewer outages over the plant lifetime, thereby reducing O\&M, fuel, and as-low-as-reasonably-achievable (ALARA) costs and personnel exposure, while increasing the observed capacity factors (Kuznetsov 2008; Carelli, Petrovic, and Mycoff 2007). Optimizing the maintenance practices and "streamlining" fuel services will also increase the capacity factor and improve plant economy (Kuznetsov 2008; Carelli, Petrovid, Cavlina, and Grgic 2005). A major downside to the extended fuel cycle is significantly reduced fuel utilization, which will impact the fuel costs.

Many design features can both positively and negatively affect the safety and capital costs. Some typical design features and their safety and cost effects are shown in Table 2 (IAEA 2009). The integral design, for instance, requires increased materials, such as concrete, to create a larger pressure vessel to hold the additional components. This negatively impacts the cost, while the elimination of large-break loss of coolant accident (LOCA) and other initiating events positively impact the capital costs (IAEA 2009).

Table 2. Pressurized water SMR design features and their targeted safety improvements and economic effects (IAEA 2009).

\begin{tabular}{|c|c|c|c|}
\hline $\begin{array}{c}\text { SMR Design } \\
\text { Feature }\end{array}$ & Targeted Safety Feature & Positive Cost Effects & Negative Cost Effects \\
\hline Modular design & - & $\begin{array}{l}\text { Decreased costs due to } \\
\text { compact design and } \\
\text { easier onsite assembly; } \\
\text { effects of mass } \\
\text { production economies }\end{array}$ & $\begin{array}{l}\text { May increase O\&M } \\
\text { costs when compared to } \\
\text { loop-type plants }\end{array}$ \\
\hline Integral design & $\begin{array}{l}\text { Elimination of large } \\
\text { break LOCA and } \\
\text { inadvertent control rod } \\
\text { ejection; larger coolant } \\
\text { inventory and thermal } \\
\text { inertia; reduction in core } \\
\text { damage frequency } \\
\text { (CDF) and large early } \\
\text { release frequency } \\
\text { (LERF) }\end{array}$ & $\begin{array}{l}\text { Reduced cost and siting } \\
\text { area due to compact } \\
\text { nature of design; reduced } \\
\text { O\&M costs with fewer } \\
\text { components and simpler } \\
\text { operations; reduced } \\
\text { safety system capital } \\
\text { costs due to } \\
\text { simplification; potential } \\
\text { for higher capacity } \\
\text { factors and longer } \\
\text { lifetime; and reduced } \\
\text { emergency planning } \\
\text { zone and security }\end{array}$ & $\begin{array}{l}\text { Increased capital costs } \\
\text { for larger reactor } \\
\text { pressure vessel increased } \\
\text { the cost due to limited } \\
\text { power output (reduced } \\
\text { neutron economy) }\end{array}$ \\
\hline $\begin{array}{l}\text { Natural circulation } \\
\text { for core heat } \\
\text { removal }\end{array}$ & $\begin{array}{l}\text { Elimination of loss of } \\
\text { flow accidents (LOFA) }\end{array}$ & $\begin{array}{l}\text { Potential for reduced } \\
\text { emergency planning } \\
\text { zone and security }\end{array}$ & - \\
\hline $\begin{array}{l}\text { Low core power } \\
\text { density }\end{array}$ & $\begin{array}{l}\text { Larger thermal hydraulic } \\
\text { margins }\end{array}$ & - & $\begin{array}{l}\text { Reduction in neutron } \\
\text { economy, increasing } \\
\text { specific operational cost }\end{array}$ \\
\hline $\begin{array}{l}\text { Removal of liquid } \\
\text { boron reactivity } \\
\text { control system }\end{array}$ & $\begin{array}{l}\text { Eliminated inadvertent } \\
\text { reactivity insertion due } \\
\text { to boron dilution; } \\
\text { reduction in } \\
\text { sabotage/human error } \\
\text { probability }\end{array}$ & $\begin{array}{l}\text { Reduced capital costs } \\
\text { due to simplification of } \\
\text { design }\end{array}$ & $\begin{array}{l}\text { Increased costs resulting } \\
\text { from changes in fuel } \\
\text { cycle properties }\end{array}$ \\
\hline
\end{tabular}


Many SMR designs are being developed, but the following represent those with designs significantly underway: advanced pressurized water, advanced boiling water, advanced heavy water, high-temperature gas-cooled (HTG-SMR), and liquid metal-cooled fast SMRs. In general, the reactor types may be grouped into categories based on the coolant used. Ultimately, the applications for all the water-cooled reactors are similar in terms of the output capacities and temperatures. Furthermore, studies on the cost correlation between different reactor designs demonstrate that there is high correlation between designs utilizing the same coolant.

Water-cooled reactor designs, including pressurized light and heavy water reactors and boiling water reactors, are analyzed in more detail throughout this document and are grouped into one Generation III+ Advanced water-cooled reactor category. Table 1 demonstrates that all the U.S. designs in more advanced stages fall into this category. However, it should be noted that high temperature reactors, such as HTG-SMRs and liquid metal cooled SMRs, have different properties and situations that would make them more favorable, such as producing high-temperature industrial process heat in addition to electricity. Thus, a brief description of each is provided in the following subsection, including the applicability of the model presented in this report.

\subsection{High Temperature Gas-Cooled SMRs}

Very few high temperature gas-cooled reactors have been operated. Figure 3 shows all historic HTGRs, most of which operated between 1965 and 1989 in the United Kingdom, United States, and Germany (IAEA 2006, PNNL 2011).

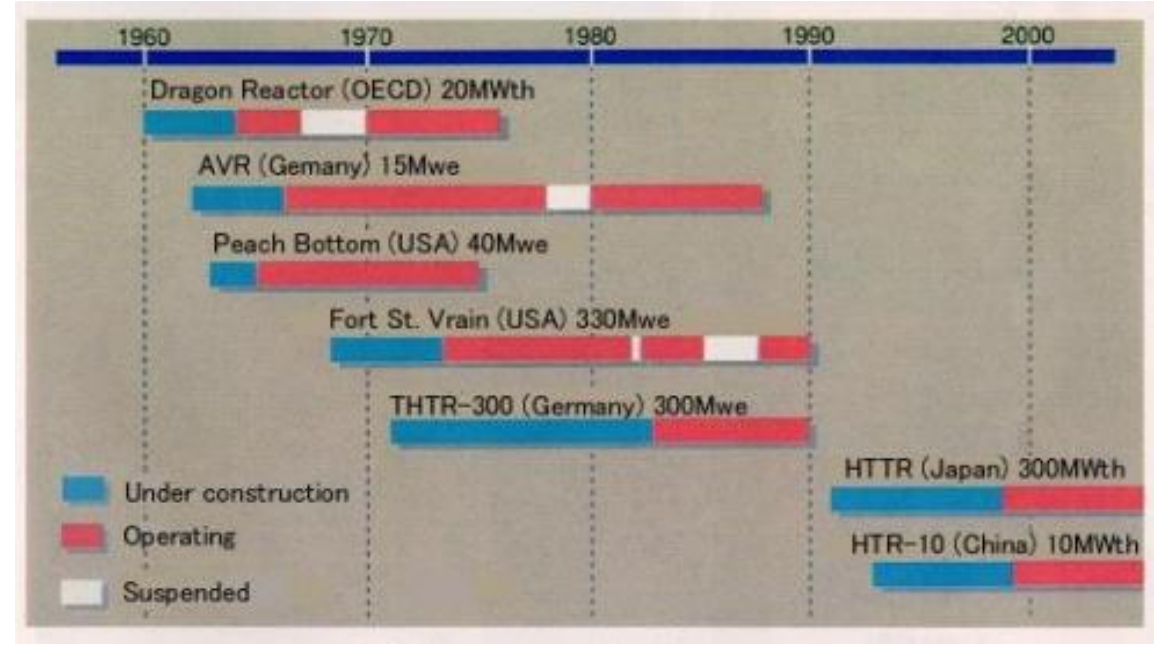

Figure 3. HTGR past reactors (PNNL 2011).

The High Temperature Engineering Test Reactor (HTTR) and High Temperature Reactor (HTR)-10 experimental reactors in Japan and China, respectively, provide the only recent operational information for this particular reactor design. Because these are experimental reactors, it is difficult to obtain the information required to estimate capital costs for an SMR. Additionally, the economic methodology detailed in this paper may not be applicable, as most HTGRs are already of small or medium size. The HTR-10 for instance only produces 10 MWth and largest High Temperature Gas-Cooled Reactor (HTGR) to operate historically had a rated power of $330 \mathrm{MWe}$, which would be considered a small reactor. HTGRs typically have a max rated power of 600 MWth (NEA and OECD 2011). The major difference between these previously operated small reactors and an SMR is the assumption that the components involved are modular and may be mass manufactured and assembled onsite. Furthermore, as design simplifications are incorporated into the modular design factor, it is assumed that passive systems and 
other methods of simplification are involved in the HTG-SMR designs. Passive decay heat removal is one such system that is commonly included (NEA and OECD 2011).

HTGRs generally have a 60-year lifetime with normal refueling periods for pin-in-block designs and a 35-40 year lifetime with online refueling for pebble-bed designs (NEA and OECD 2011). Thus, the capacity factor for a pebble bed design would be much closer to $100 \%$ than that of a pin-in-block design. HTGRs utilize tristructural isotropic (TRISO) fuel kernels, which is why they may operate up to significantly higher temperatures than LWRs. In the pin-in-block design, the fuel elements are immobile in a graphite matrix as fixed fuel pins. In a pebble-bed design, the fuel is embedded in a graphite sphere that is free to circulate the core (NEA and OECD 2011). Unlike other designs, they operate at much higher temperatures, typically $800-900^{\circ} \mathrm{C}$ (NEA and OECD 2011). As a result, the rejected heat may be used for cogeneration via an intermediate heat exchanger. Figure 4 shows the various temperatures in which different cogeneration processes occur, demonstrating how the high temperatures produced in HTG-SMRs would be useful for high temperature industrial process heating, such as hydrogen production via electrolysis (NEA and OECD 2011; INL NGNP).

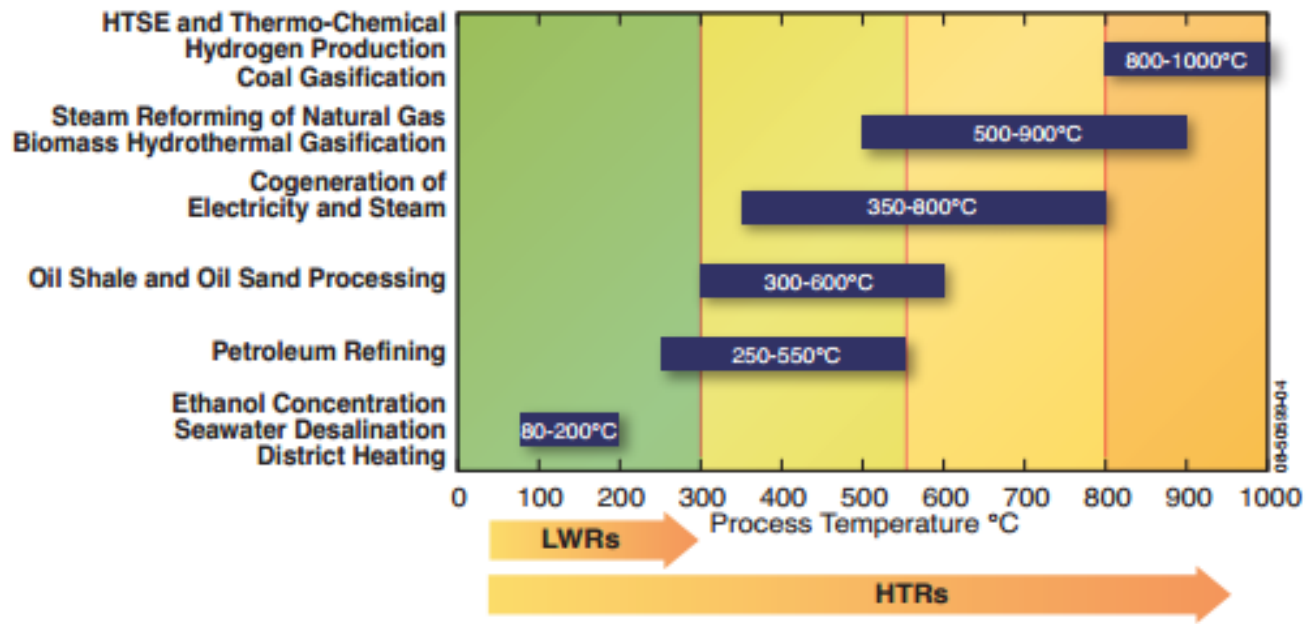

Figure 4. Cogeneration process temperatures (INL NGNP).

\subsection{Liquid Cooled Fast Reactors}

Lead-bismuth cooled fast SMR designs being developed assume that the components and fuel are both factory-fabricated and that refueling occurs in 7-20 year intervals. The capacity factor over the plant lifetime of 50-60 years is determined as 95\% (NEA and OECD 2011). The designs use compact pressure vessels, which may be modularly assembled. The total construction time for such a plant is 1.75-3.5 years, falling in line with the 3 -year estimates used in the economic methodology presented in this report (NEA and OECD 2011). There are some major concerns with using this type of reactor. It may only be operated at lower temperatures to control corrosion of the fuel cladding and structural components (IAEA 2007b). Furthermore, the $\mathrm{Pb}-\mathrm{Bi}$ coolant must be continually heated to maintain its temperature above the melting point of $125^{\circ} \mathrm{C}$ so that it does not solidify. Irradiated bismuth will produce Polonium-210, which is volatile and must be carefully monitored (NEA and OECD 2011).

Furthermore, lead-bismuth cooled fast reactors have never been operated in any country. Russia used the same coolant for propulsion reactors, but never in fast reactors. This, along with the corrosion and production of Po-210, would make it difficult for a reactor of this type to obtain licensing, particularly in the U.S. Finally, there is no relevant cost information to use as a reference point in an economic study. Additionally, with the extremely limited historical operational context, the applicability of the economic methodology in this report is unclear. 
Sodium-cooled fast reactors have been operated successfully in the past, and the last one shut down in 2010 (NEA and OECD 2011). They have been used for electricity generation, breeding, and desalination. The BN-350 plant operated for its entire design life over 25 years, producing 100,000 tons of desalinated water per day and $150 \mathrm{MW}$ electricity (IAEA 2007). Other plants such as the French Phenix and Super-Phenix and the United Kingdom's Prototype Fast Reactor also successfully operated. The Super-Phenix reactor of 1200 MWe was the first large reactor of this design (IAEA 2007). Sodium performs very well as a coolant under high heat generation rates. However, an intermediate heat exchanger is always required, so that the sodium never contacts air or water, as it will react violently (NEA and OECD 2011). These reactors may also be utilized for cogeneration, as they operate at temperatures above $500^{\circ} \mathrm{C}$. With prior operation of sodium-cooled fast reactors, it may be easier to obtaine the necessary cost information to follow the methodology presented in this report.

\subsection{ECONOMIES OF MASS PRODUCTION}

The modularization of SMR units provides a unique opportunity for factory fabrication of standardized modular units that may be transported to a site and assembled rapidly, decreasing construction costs while increasing learning (Kuznetsov 2008). It also lays the framework for ordering modules in bulk, thereby reducing costs via economies of mass production or replication (Carelli, Petrovic, and Mycoff 2007). The effects of modularization become more apparent with each additional unit produced and installed up to a certain point in which the effects level off, as shown in Figure 5 (NEA and OECD 2011; Mitenkov et al 2004). The first unit produced sees an approximately $15 \%$ reduction in costs, the second, third, and fourth units see a 5\% reduction in costs, and subsequent units see limited reductions (Wilton 2012). Therefore, NOAK modularized and mass-produced units would see a maximum reduction of approximately $35-40 \%$ from the FOAK unit.

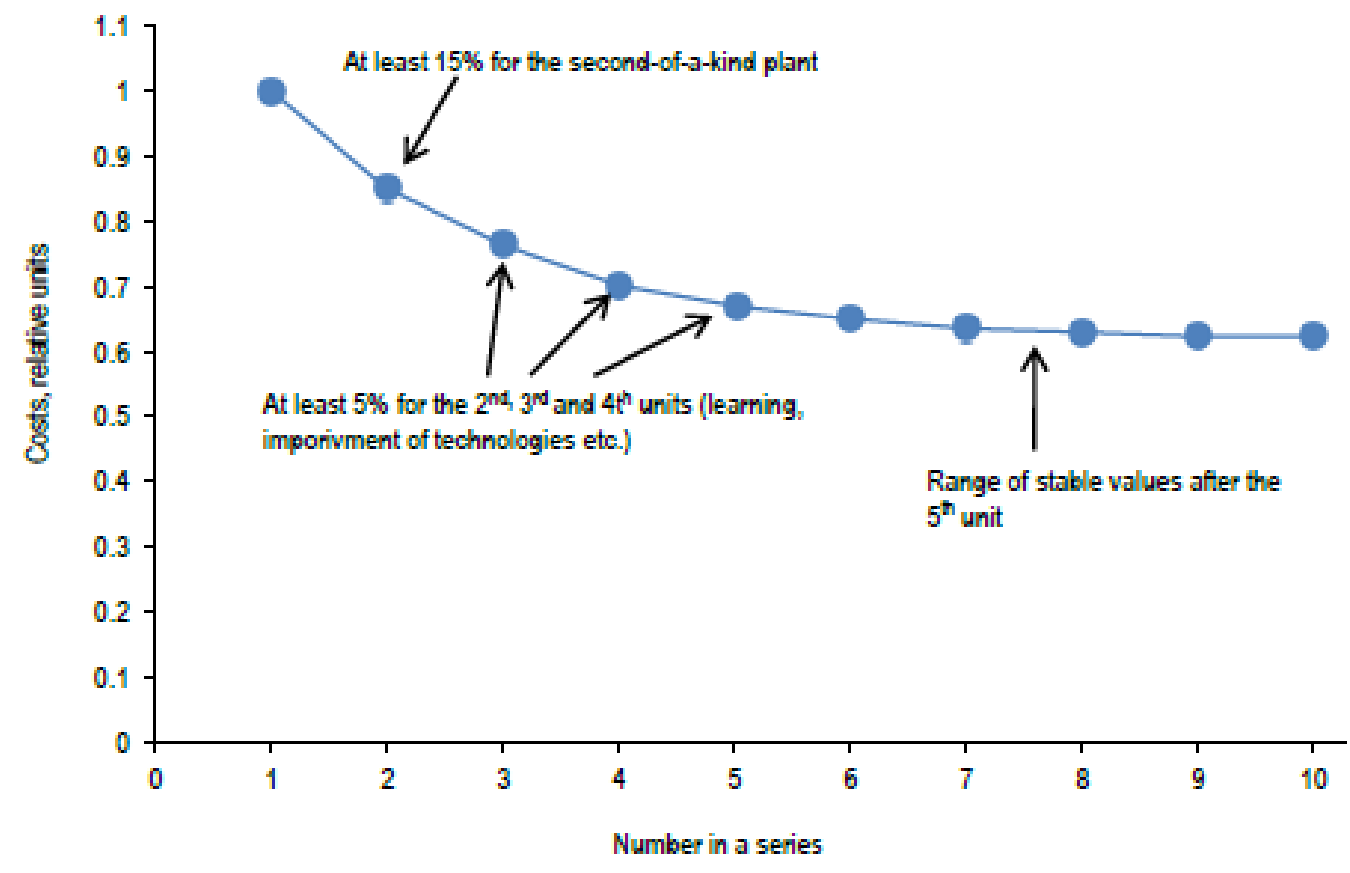

Figure 5. Serial production effects for nuclear propulsion reactors (Mitenkov et al. 2004).

\subsection{CONSTRUCTION AND TIMING}

Construction for SMRs is expected to take less time than that of a large reactor. The reduction from 5-6 years of construction to 3-4 years due to modular construction and limited onsite assembly will affect 
the capital costs due to the interest during construction (Locatelli, Bingham, and Mancini 2014; NEA and OECD 2011; Wilton 2012). In this economic study, a 6-year construction period for large reactors is assumed, given that the actual construction times to grid connection or commercial generation vary significantly, as shown in Figure 6, and the U.S. construction times have nearly doubled since the Three Mile Island incident (Schneider, Frogatt, and Hazemann 2012).

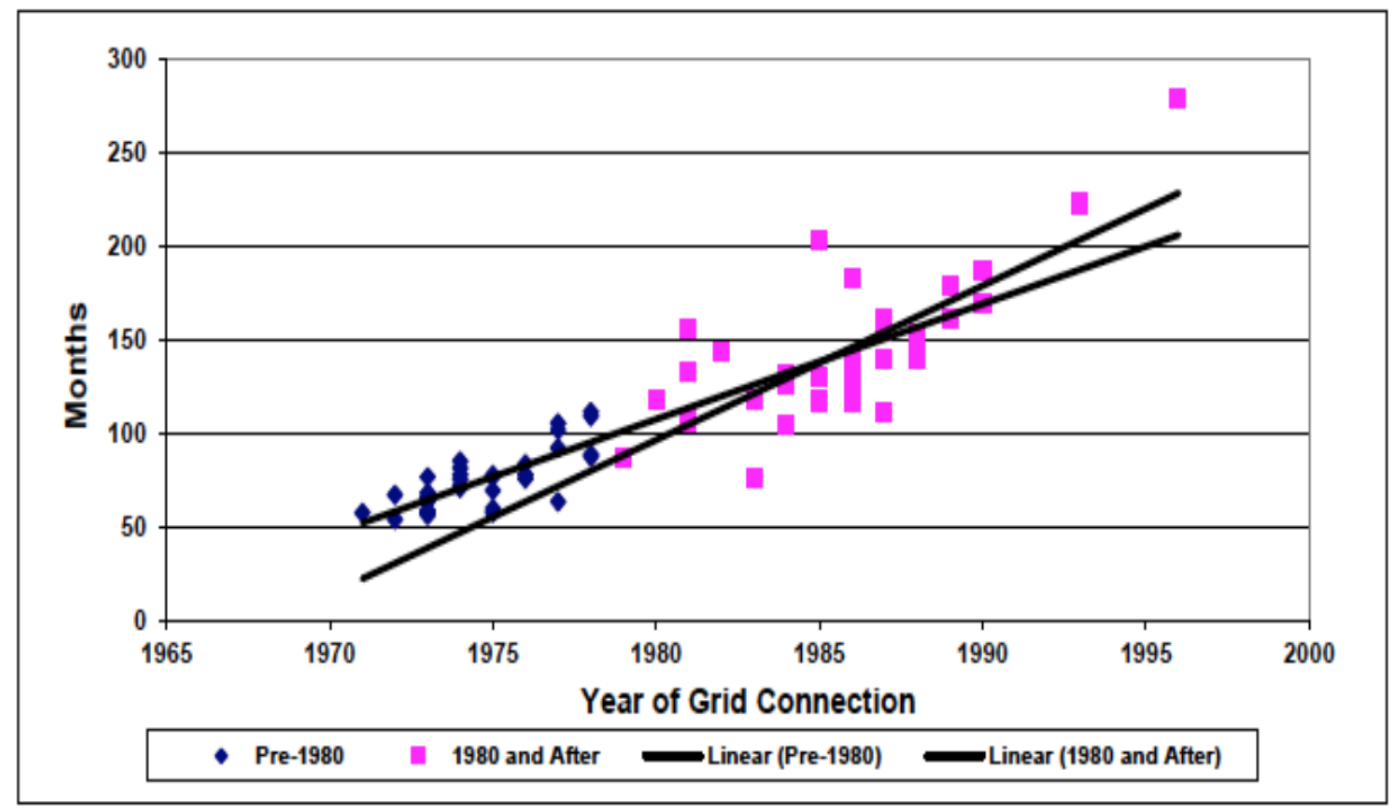

Figure 6. United States large reactor construction times in months (Schneider, Frogatt, and Hazemann 2012).

Testing required once construction is complete is another important factor and in the past have been longer than the entire construction of fossil fuel plants. For example, Units 1 and 2 at the Vogtle NPP required 50 months of testing prior to normal operations (INL 2002). A testing schedule may be a valuable addition to an economic model.

Timing or deployment schedule may help mitigate the effects of high upfront capital costs. Different deployment scenarios in which only one plant is constructed at a time are explored in this economic model, allowing for self-financing of subsequent units (Carelli, Petrovic, Cavlina, and Grgic 2005). This also allows the upfront capital for the project to be borrowed at intervals, thereby reducing the interest during construction.

Construction efficiency is greatly impacted by the climate in which the construction takes place. For example, the early phase in French nuclear development during the 1970s to early 1980s had a mean construction time that was approximately $30 \%$ less than the mean construction times observed for U.S. plants (Grubler 2010). The major differences between the environments were the standardization of French reactors, regulatory stability, centralized oversight, and client engineering (Grubler 2010). On the other hand, in the U.S., the environment may be considered decentralized, in the sense that both state and federal governments play a part. Additionally, the political environment may suddenly change, producing legislation or laws that slow construction and development of nuclear plants. . The effects of the political climate are not directly included in the economic model presented, but it should be clear that the effects can greatly increase overall construction times and costs and may inhibit the optimal timing and deployment of units. 


\subsection{PLANT CONFIGURATION}

Plant configuration may play a significant role in capital costs. The development of twin and multi-module plants affect the costs associated with sharing systems and structures. These shared structures should be maximized for twin units. Unfortunately, this means that additions to a site must be made two units at a time (Carelli, Petrovic, Cavlina, and Grgic 2005). Another important configuration is multi-module clustering, in which space, equipment, facilities, etc., are set aside for future development (IAEA 2006). ${ }^{\text {Error! Reference source not found. }}$ For example, the switch yard may be initially constructed for four nits, even though two units are only initially constructed. The next two units may be constructed as electricity demand grows. The capital costs for these additional units will then be shared with the previous two units who have been providing income from operations.

\subsection{LEARNING}

Learning occurs upon the development and construction of similar units over time and may be applied to manufacturing, construction and assembly, and operations and maintenance. Several different views exist on the effects of learning, but all agree qualitatively that with each subsequent unit built, installed, and operated the personnel involved gain experience. For example, the construction crew should better understand the installation of the fifth identical unit when compared to the first unit on the same site. Rosner and Goldberg state that the learning rate for fixed capital costs and for variable operational costs are $10 \%$ and $2-3 \%$, respectively, implying that with each subsequent unit, a cost reduction may be applied (Rosner and Goldberg 2011). The Nuclear Energy Agency details how the learning effects vary for the FOAK SMR and each subsequent unit (single SMR, twin SMR units, or multi-module units) based on construction learning, factory fabrication learning, and learning due to sharing of facilities and systems on the site (NEA and OECD 2011). The EMWG describe a learning rate of 3-4.5\% as each doubling of rated power that occurs from FOAK to NOAK (Generation IV EMWG 2006; Rothwell 2007).

Wilton provides a more exhaustive method of determining the learning effects for each unit, based on experience from all plants both onsite and internationally of the same type; breakdown of the equipment, labor, and material cost percentages; and the equipment, labor, and material learning rates. The sum of each unit's learning factor is then the total learning rate (Wilton 2012). The learning rate is sometimes determined to be greater than unity, meaning an increase in the capital cost will occur. This may be attributed to the negative learning that occurs when additional safety features and procedures to improve operations are deemed necessary based on experience. Any safety enhancements and features will likely increase the capital and O\&M costs (Grubler 2010). Negative learning may also occur during the construction period if the utility or principal agent and the engineering, architectural, construction, etc. firms are not working together effectively (Grubler 2010). For this economic study, it is assumed that learning increases with each unit, resulting in reduced investment and O\&M costs. 


\section{ECONOMIC ANALYSIS METHODOLOGY}

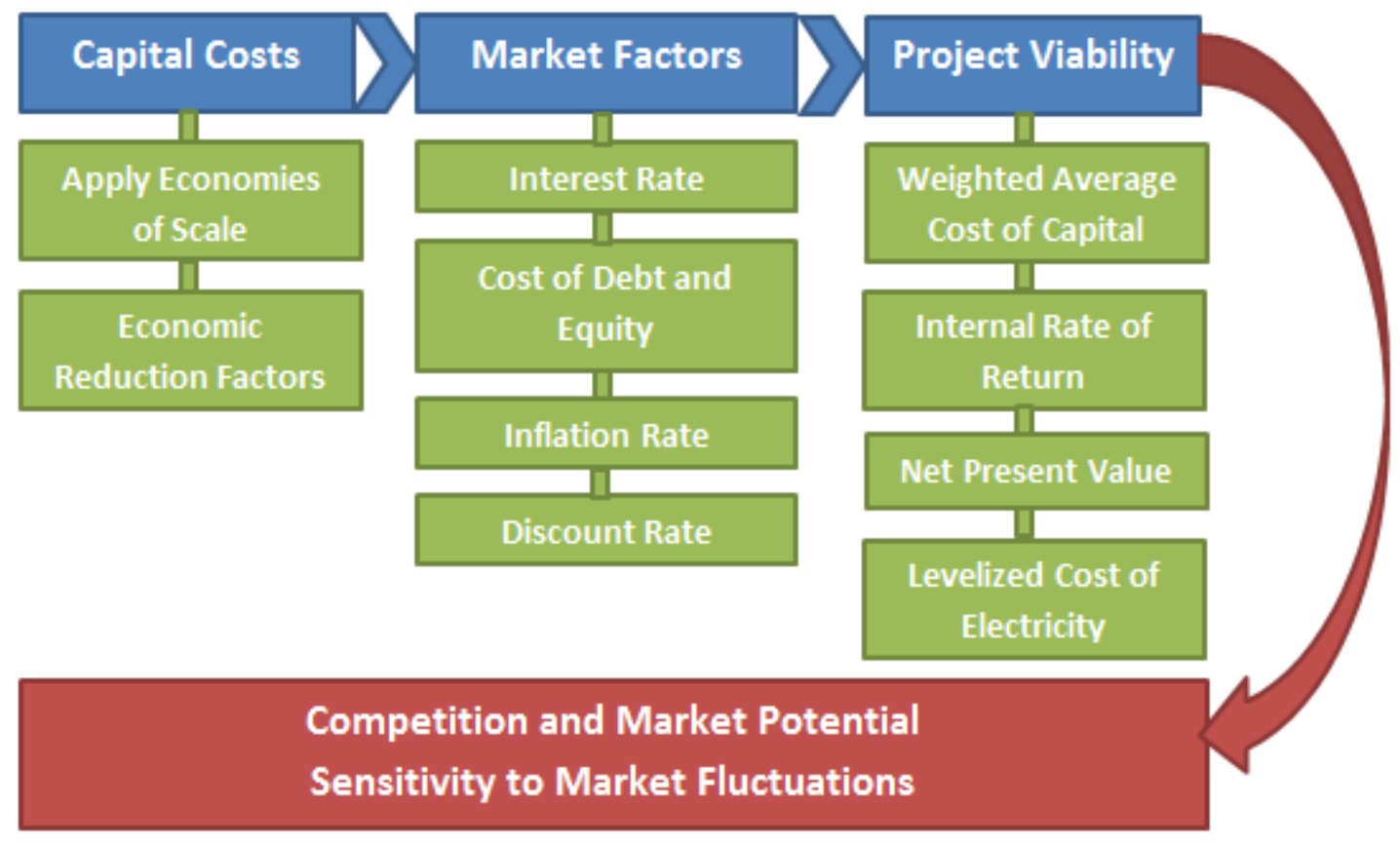

Figure 7. Overview of Economic Analysis Methodology.

\subsection{Estimating OCC for SMRs from LR Data}

Equations 1 and 2 demonstrate how to calculate the SMR specific and total capital cost, respectively, by utilizing the economies of scale curve from Figure 1 (NEA and OECD 2011; IEA 2000). This, in effect, scales the capital costs as a function of rated power. In these equations, $P_{0}$ and $P_{1}$ are the large reactor and SMR rated power in MWe, respectively. $\eta$ represents the scaling factor, which is reported to range from 0.4-0.7 dependent on the specific SMR project (NEA and OECD 2011). Carelli et al. determined a mean scaling factor as 0.6 (Wilton 2012; Carelli et al 2010). The Nuclear Energy Agency determined an average scaling factor of 0.51 , which is used in the calculations for this report (NEA and OECD 2011). It should be noted that these equations are typically applied in the energy range from 300 MWe to 1300 MWe (NEA and OECD 2011). Many SMRs fall below this range, increasing the uncertainty in this calculation. Additionally, these equations are meant to scale the costs between reactors without any design changes, which is why the large reactor selected for comparison as in Table 1 and the design factors applied and later discussed are significant to the economic study.

$$
\begin{aligned}
& \frac{\operatorname{Cost}\left(P_{1}\right)}{P_{1}}=\frac{\operatorname{Cost}\left(P_{0}\right)}{P_{0}}\left(\frac{P_{1}}{P_{0}}\right)^{\eta-1} \\
& \operatorname{Cost}\left(P_{1}\right)=\operatorname{Cost}\left(P_{0}\right)\left(\frac{P_{1}}{P_{0}}\right)^{\eta}
\end{aligned}
$$

The costs obtained from Equations 1 and 2 are then adjusted based on factors for the development, site usage, and construction of a particular SMR project. The reduction factors are detailed in sections 11.1.1-11.1.4.

\subsubsection{Co-Siting Reduction Factor}

When multiple units are constructed on one site, or co-sited, a reduction in capital cost, as certain indivisible costs are distributed upon multiple units. There is a proportion of direct costs $F_{D I R}$ which is 
applied to each unit as well as a proportion of indivisible costs $F_{I N D}$ which only apply to the first unit. Equation 3 describes how the Multiple Units Factor is calculated for $n$ units (Wilton 2012). Carelli et al. provide typical values for $F_{I N D}$ and $F_{D I R}$ of 0.34 and 0.66 , respectively (Carelli et al. 2010). It should be noted that as $n$ increases, the Multiple Units Factor approaches $F_{D I R}=1-F_{I N D}$, and the indivisible costs are effectively eliminated.

$$
\text { Co }- \text { siting Factor }=\frac{1+(n-1)\left(1-F_{I N D}\right)}{n}
$$

\subsubsection{Learning Reduction Factors}

Learning may be broken down into two categories: plant configuration and technological maturity. Multiple, modular units result in increases in plant configurational learning from fabrication, construction, and sharing of facilities and systems. Based on the particular plant configuration, such as twin or individual units, the sharing of facilities and systems will vary. The Nuclear Energy Agency method for calculating learning involves determining values for four variables representing differences in costs for the first unit and additional units with shared systems and facilities, where multi-module facilities have the maximum shared facilities and learning. Equations 4-6 represent the plant configuration reductions for the first unit, twin units, and multi-module units, where $x$ represents the FOAK extra cost factor (15$55 \%), y$ represents the gain for a pair of twin units (74-85\%), $z$ represents the gain in building two pairs of twin units on the same site ( $82-95 \%)$, and $k$ represents the industrial productivity coefficient (0-2\%) (NEA and OECD 2011; IEA 2000).

For the FOAK unit, the learning factor simply becomes $1+x$ and increases the total capital. For the first and second pairs of twin units, the learning factor per unit may be calculated from Equations 4 and 5, respectively. The total learning factor per unit for this site of four twin units is then determined in Equation 6 and falls in the range of 0.81 to 0.9 . For a 5+ multi-module plant, no data can be determined from prior experiences with reactors; however, it may be assumed that a $15-17 \%$ reduction of the learning factor for a twin unit site is observed (NEA and OECD 2011).

$$
\begin{aligned}
& \text { Learning Factor First Pair }=\frac{1+y}{2} \\
& \text { Learning Factor Second Pair }=\frac{1}{2}\left(\frac{z}{1+k}+\frac{y}{(1+k)^{2}}\right) \\
& \text { Learning Factor Site }=\frac{1}{4}\left(1+y+\frac{z}{1+k}+\frac{y}{(1+k)^{2}}\right)
\end{aligned}
$$

Wilton provides an alternative method to calculating the learning factor, as shown in Equation 7, where $f$ is the total number of units on-site and $c_{n}$ is the cost escalation rate (Wilton 2012; Carelli et al. 2010). $C_{e q}, C_{l a b}$, and $C_{m a t}$ refer to the equipment, labor, and material costs and may be determined from Equations 8-10, where $K_{e q}, K_{l a b}$, and $K_{\text {mat }}$ are the equipment, labor, and material percentages; $N_{\text {world }}$ and $N_{n}$ are the total offsite plants of same type and the progressive number of plants onsite; and $\alpha, \beta_{1}$, and $\beta_{2}$ are the factory equipment learning rate, production site labor learning rate, and the world labor learning rate (Wilton 2012).

$$
\begin{gathered}
\text { Learning Factor }=\sum_{n=1}^{f} \frac{C_{e q}+C_{\text {lab }}+C_{\text {mat }}}{f} * c_{n} \\
C_{e q}=K_{e q}\left(N_{\text {world }}+N_{n}\right)^{\alpha} \\
C_{l a b}=K_{l a b}\left(N_{\text {world }}+1\right)^{-\beta_{2}}\left(N_{n}\right)^{\beta_{1}} \\
C_{\text {mat }}=K_{\text {mat }}\left(N_{n}\right)^{\alpha}
\end{gathered}
$$


To use the learning factor calculated from Equations 7-10, it is necessary to estimate a number of values related to onsite and offsite learning rates and cost proportions for equipment, labor, and materials. For most SMR preliminary cost estimates, this information is unavailable and difficult to approximate. Thus, for general economic calculations, the learning factor methodology detailed by the Nuclear Energy Agency should be used, as previously described in Equations 4-6.

The Generation IV EMWG details a process of calculating the learning effects on Base Construction Costs as one progresses from FOAK to NOAK. To approximate this information, a learning of 3-4.5\% occurs for each doubling of rated power up until 8 GWe (Generation IV EMWG 2006; Rothwell 2007). This learning rate methodology follows the Learning Curve Methodology, whereby a learning rate is selected from historical data or based on the state of the technology. The National Energy Technology Laboratory reports that technology may be fully mature or immature, and the more immature or new a technology is, the higher the learning rate will be (NETL 2013). Equation (12) represents the general learning rate form, in which $Y$, the cost to produce the cumulative rated power, is $X, A$ is the FOAK cost, and $b$ is the learning rate exponent. $b$ is defined by $b=-\log (1-R) / \log (2)$, where $R$ is the learning rate.

$$
Y=A X^{-b}
$$

Typical values for the learning rate range from 0.01 to 0.06 based on maturity and are presented in Table 3 (NETL 2013). Complex systems will have components with many different learning rates. Thus, a nuclear plant should contain a mix of both mature and immature technologies. For this economic model, a $4.5 \%$ learning rate was used.

Table 3. Sample learning rates and level of maturity (NETL 2013).

\begin{tabular}{|l|c|}
\hline \multicolumn{1}{|c|}{ Maturity } & $\begin{array}{c}\text { Learning Rate } \\
\text { (R) }\end{array}$ \\
\hline Experimental_FOAK & 0.06 \\
\hline Promising & 0.05 \\
\hline Growing & 0.04 \\
\hline Proven & 0.03 \\
\hline Successful & 0.02 \\
\hline Mature-NOAK & 0.01 \\
\hline
\end{tabular}

\subsubsection{Modular Design Reduction Factor}

The final factor considered quantitatively is the modular design factor describing the reduction that occurs from the factory fabrication of reactor components. As Figure 8 shows, the modular design factor increases with increasing rated power above $35 \mathrm{MWe}$ (Reid 2003). Below $35 \mathrm{MWe}$, nearly the entire system is factory fabricated, so the factor is constant at 0.6. Equation 11 is a fit to the curve in Figure 7 as a function of rated power $P_{r}$ in MWe (Wilton 2012). 


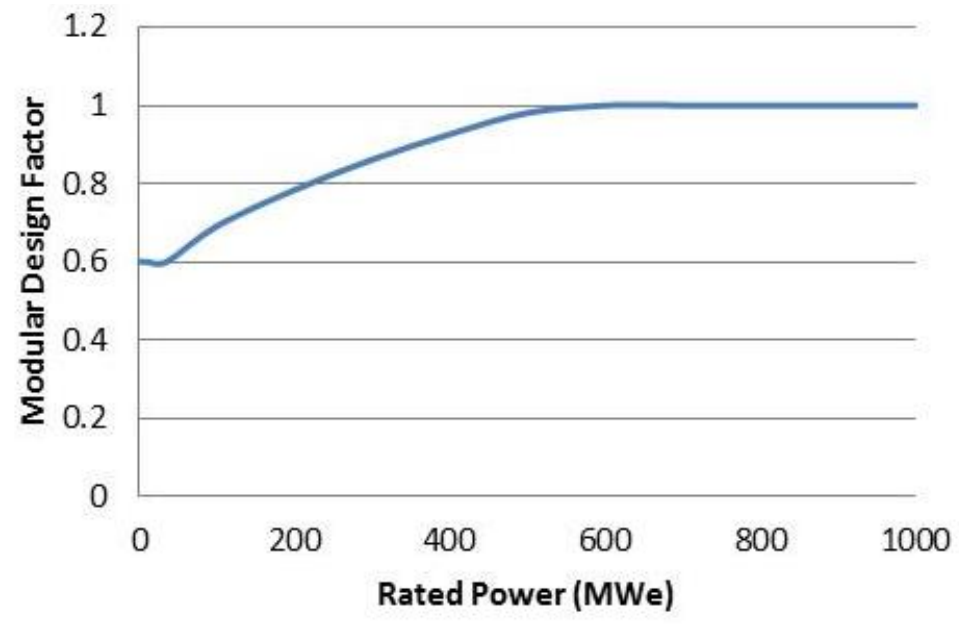

Figure 8. Effects of modularity as a function of rated power (MWe) (Reid 2003).

Modular Design Factor

$=\left\{\begin{array}{c}0.6 \text { for } P_{r} \leq 35 \mathrm{MWe} \\ 4 \times 10^{-10}\left(P_{r}\right)^{3}-10^{-6}\left(P_{r}\right)^{2}+0.0012\left(P_{r}\right)+0.581 \text { for } 35<P_{r}<600 \mathrm{MWe} \\ 1 \text { for } P_{r} \geq 600 \mathrm{MWe}\end{array}\right.$

For the design simplification that occurs in SMRs, factors of 0.84-0.85 have been reported for light water designs. This value, for example, is the same factor used for design simplification due to the integral design in several other economic studies (NEA and OECD 2011; Kuznetsov 2008; IAEA 2010). In this economic model, the design simplification costs are assumed to be incorporated into the modular design factor and are not separately utilized.

\subsubsection{Summary of Reduction Factors}

Once all of these factors have been calculated, the total and specific capital costs may be determined by scaling the large reactor capital cost and multiplying it by the co-siting, learning, and modular design factors. A summary of the economic factors and the values used are shown in Table 4.

Table 4. Summary of economic factors.

\begin{tabular}{|c|c|}
\hline Factor & \\
\hline Scaling & 0.51 Equations 1 and 2 \\
\hline Co-siting & Equation 3 \\
\hline Learning (New Technology) & $\begin{array}{l}\text { Equation } 4 \text {-FOAK Unit } \\
\text { Equation } 5 \text { - One Pair Twin Units } \\
\text { Equation } 6 \text { - Two Pairs Twin Units } \\
15-17 \% \text { Reduction of Equation } 5 \text { for Multi-Module Units } \\
\text { Equation } 11\end{array}$ \\
\hline Modular Design Factor & Equation 12 \\
\hline
\end{tabular}




\subsection{Interest During Construction}

Interest during construction (IDC) refers to the interest that must be paid on the construction cost loans and is dependent on the particular payment schedules during construction. This is an additional cost that must be included in determining the total capital cost. Equation 13 shows the general method used to calculate the IDC for short construction times only, where $N$ is the number of construction years, OCC is the overnight capital cost, and $r$ is the interest rate on the loan (INL 2002). The real discount rate may be used for the loan interest rate. Further information on determining the real interest rate may be found in Section 11.4.3.

$$
I D C=\frac{N}{2}\left[\frac{O C C}{N} *(1+r)^{N-1}-\frac{O C C}{N}\right]
$$

Equation 13 is an approximation assuming the construction costs are equally distributed between $N$ years of construction and that modal payments made are half of the interest amount (INL 2002).

\subsection{Calculating Total Capital Investment Cost}

The EMWG states that the total capital investment cost (TCIC) is "an all-inclusive plant capital cost (or lump-sum up-front cost) developed for the purpose of calculating the plant" LCOE, as detailed in Section 11.4.7. "This cost is the base construction cost plus contingency, escalation, IDC, owner's cost (including utility's start-up cost), commissioning (non-utility start-up cost), and initial fuel core costs (for reactor)" (Generation IV EMWG 2006).C. L. Kling and M. D. Carelli, "IRIS Licensing Status," IEA/INIS, 2014. [Online]. Available: http://inis.iaea.org/search/search.aspx?orig_q=RN:44064691.9 The TCIC incorporates the OCC and IDC costs. For this economic study, the TCIC may be approximated from Equation 14, where $D D \& E$ refers to detailed design and engineering costs, $C C$ refers to Contingency Costs (see Section 12.1), and IDC refers to Interest during Construction.

$$
T C I C=D D \& E+O C C *(1+C C)+I D C
$$

\subsection{Market Factors and Project Viability}

Once the capital costs are calculated for a particular SMR project, it is necessary to assess the market conditions and project viability. This is done by determining the IRR, NPV, and LCOE and comparing them to the weighted average cost of capital (WACC) and expected electricity prices.

\subsubsection{Market Types}

The specific type of market present in a region will dictate the conditions in which nuclear power is competitive. In a regulated utility market, "generating plants operate under cost-of-service rate regulation and have market outlets for the electricity within the same company" (WNA). This model, which is generally assumed for the electricity market, provides less investment risk, as the investor's costs may be charged to the customers as part of the electricity price - if regulators agree to this (WNA). In an unregulated merchant market, "generating plants compete and have no direct outlets for selling electricity" (WNA). This is, in essence, a liberalized market, which has increased risks. To mitigate these risks, power purchase agreements or other methods may be used. Additionally, the company typically must have a higher share of equity over debt (WNA).

Hybrid markets "consist of some merchant generating plants but evolve towards a small number of vertically integrated large utility groups, with a spread of generation facilities and regional supply outlets" (WNA). Finally, the investment market is one in which "nuclear is now attractive to utilities previously not involved in the sector. These are likely to participate via long term partnership agreement for building and operating nuclear plants, typically with other companies more experienced in the business" (WNA).

The economic models presented within this report do not directly the particular market setting into account, in an effort to make the model more widely applicable. 


\subsubsection{Weighted Average Cost of Capital}

Costs of equity and debt refer to a company's opportunity cost of investing in a particular project rather than in another investment with similar risk. The primary difference between debt and equity is the risk involved in borrowing. Equity borrowing is riskier than debt borrowing, as it necessitates leveraging assets to fund the investment (INL 2012). Assessing appropriate values for equity and debt in the nuclear industry is difficult due to large variations in risks as a result of construction and regulatory overruns and delays, which may translate into higher opportunity costs for equity and debt. In this economic model, the Weighted Average Cost of Capital (WACC) is calculated and then used to determine the appropriate discount rate.

The WACC is a value describing the percentage of capital that must be paid to the investors so they see the expected return on investments/assets. The WACC may be affected by many factors, such as political and financial risks, which are further discussed in the results and discussion portion of the paper. Equation 15 details how WACC was determined, with $C_{D}$ and $C_{E}$ representing the costs of capital and equity, respectively, $T_{C}$ representing the corporate tax rate, and $D$ and $E$ representing the portions of debt and equity for the project (IEA 2010). The cost of debt is the opportunity cost of investing in the project or the interest rate. The cost of equity is also a factor of the interest rate or opportunity cost, but is also affected by the corporate tax rate. It may be calculated by $K_{E}=K_{D} *\left(1+T_{C}\right)$. Debt interest payments are considered tax deductible in the U.S. Thus only the cost of equity reflects taxation (INL 2012). The average share of debt for large reactor projects is $65 \%$. This value for SMRs is approximately $50 \%$ (Carelli 2008; INL 2012). The maximum share of debt for large reactors and SMRs are approximately 93\% and 69\% respectively (Carelli 2008). In this economic model, the cases are performed at the average SMR debt share. Sensitivity studies are also performed at 30\% and $70 \%$ debt shares.

$$
W A C C=D * C_{D}+E * C_{E}
$$

\subsubsection{Real and Nominal Discount Rate Methods}

The discount rate is an interest rate that provides the current worth of future money. A nominal discount rate includes inflation, while the real discount rate does not. Either one may be used in evaluation the net present value (NPV), but different processes must be used. Nominal discount rates typically fall between $5 \%$ and $10 \%$ for regulated and deregulated markets, respectively (Generation IV EMWG 2006). The real discount rate $d_{r}$ may be calculated from the WACC and the inflation rate $r_{i}$ as in Equation 16. The relationship between the real and nominal discount rates may be approximated from Equation 17 for long-term investments (Short, Packey, and Holt 1995). More information on determining an appropriate discount rate may be found in Appendix B.

$$
\begin{gathered}
d_{r}=\frac{W A C C+1}{r_{i}+1}-1 \\
d_{d}=d_{r}+r_{i}
\end{gathered}
$$

It may be appropriate to use multiple interest rates in assessing the economic lifetime for a nuclear plant, as the risks associated with such an investment are drastically different during construction and normal operations. Once construction of the plant and infrastructure is completed and with the operating license granted, the risk of the venture decreases (INL 2012). The risk will continue to decrease the longer the plant operates to a certain extent. For this economic analysis, a single real discount rate was applied.

\subsubsection{Fixed Charge Rate and Amortization of Capital Costs}

A simplified calculation of the Fixed Charge Rate (FCR) as in Equation 18 based on the real discount rate may be performed to levelize the capital costs over the plant lifetime $t_{\text {life }}$. The FCR becomes an amortization factor as in Equation 19 if depreciation, taxes, and capital return are not considered. 


$$
\begin{gathered}
F C R=\frac{d_{r}}{\left[1-\left(1+d_{r}\right)^{\left.-t_{\text {life }}\right]}\right.} \\
\text { Annual Cost }=\text { TCIC } * \text { FCR }
\end{gathered}
$$

\subsubsection{Net Present Value}

The total NPV is the sum of the present values of all annual cash flows, or the discounted value of all annual cash flows. It may be calculated from Equation 20, where $C F_{y}$ represents the annual cash flow during year $y$ and $n$ is the total number of years including construction and operating lifetime. More information on calculating NPV may be found in Appendix C.

$$
N P V=\sum_{y=0}^{n} \frac{C F_{y}}{\left(1+d_{r}\right)^{y}}
$$

\subsubsection{Internal Rate of Return}

The IRR is a project performance term that describes the return on investment over the project lifetime. It is determined by iterating until the NPV becomes zero at a discount rate equal to the IRR. For a firm to pursue a project, the IRR should be greater than the WACC. The larger the gap between IRR and WACC, the higher the profits and the return on initial investment. Equation 21 details how the IRR is calculated. More information on calculating the IRR may be found in Appendix D.

$$
N P V=\sum_{y=0}^{n} \frac{C F_{y}}{(1+I R R)^{y}}=0
$$

\subsubsection{Levelized Cost of Electricity}

"For any investment in nuclear projects, the revenue generated by the sale of the product needs to cover both the fixed and variable expenses incurred during normal operations; to repay the capital employed during the construction and decommissioning phases, including both overnight and financing charges; and to compensate the owners of the capital (both debt and equity investors) for the risk taken with the project. The electricity price that, in real dollars, covers all these charges is called 'Levelized Cost of Electricity'” (INL 2012).

The LCOE represents the cost of energy production averaged over the lifetime of the plant, as shown in Equation 22. In this case, the discounted cost cash flow includes capital, O\&M, fuel, and decommissioning costs discounted at the real or nominal discount rate. The discounted annual energy represents the annual electricity production discounted at either the real or nominal discount rate. The former will provide the real LCOE, while the latter will yield the nominal LCOE. The real LCOE is used in this report. Additional information on determining the LCOE may be found in Appendix E (Generation IV EMWG 2006).

$$
\text { LCOE }=\frac{\sum_{y=0}^{n} \text { Discounted Annual Cost Cash Flow }}{\sum_{y=0}^{n} \text { Discounted Annual Energy }}
$$

\section{ECONOMIC ANALYSIS INPUTS}

The majority of the economic cases detailed in this paper assume the reactors to be of an Advanced Generation III+ design, which is meant to incorporate both Pressurized and Boiling Water reactor designs. Advanced Pressurized Water Reactors, such as the Westinghouse AP1000 1200MWe reactor, are not yet in operation (WNA 2014). As a result, the only operational Advanced Generation III+ reactorsABWRs - were used to represent all advanced LWRs (Hitachi-GE Energy Ltd). When other advanced LWR designs are constructed and begin operation, then more accurate capital costs and construction 
times may be used for an improved SMR economic model. Appendix F contains the Excel SMR model inputs.

\subsection{DD\&E and Contingency Cost}

No specific DD\&E costs are available for SMR designs, as companies wish to maintain these as private records during early phases of development. However, Rosner and Goldberg estimate that \$0.8-\$1 billion is a conservative estimate to develop a new SMR design that will meet Nuclear Regulatory Commission requirements, provide for construction cost estimates of the first SMR plant, and provide for the design estimates required to build a manufacturing plant specific to the SMR (Rosner and Goldberg 2011). Contingency costs arise from changes in the political, regulatory, financial, etcetera conditions during construction. The Nuclear Energy Agency states that this may increase capital costs for large nuclear reactors by $5-10 \%$ internationally and by approximately $5 \%$ in the United States (IEA 2010). It is assumed that this same percentage may also be applied to NOAK SMRs. FOAK SMRs, on the other hand, are assumed to have contingency costs of 15-55\% (NEA and OECD 2011).

\subsection{OCC, O\&M, and Fuel Costs}

A 1350 MWe Advanced Generation III+ Light Water Reactor with a 90\% capacity factor was used to determine the specific and total OCC associated for a scaled water-cooled SMR (NEA and OECD 2011). The total OCC of $\$ 4.57$ billion was used. No large reactor capital costs are available for HTG-SMRs as previously mentioned. Current SMR designs under development claim to have specific capital costs under $\$ 1,700 / \mathrm{kWe}(\mathrm{NEA}$ and OECD 2011).

O\&M costs have been approximated by many sources for large reactors. For instance, the Nuclear Energy Agency's mean O\&M costs for large reactors were determined to be $\$ 14.66 / \mathrm{MWh}$ (NEA and OECD 2011). Combined O\&M and fuel costs have been approximated by several sources with ranges of \$7.1-\$36.2/MWh for SMRs with longer refueling periods (NEA and OECD 2011). The higher end of this range is more likely, as the fuel utilization and economy is much lower for longer refueling periods. If conventional refueling periods are used, then the combined costs are approximately $\$ 7.1-\$ 26.7 / \mathrm{MWh}$ (NEA and OECD 2011). Rosner and Goldberg estimate the fuel and O\&M costs for a $100 \mathrm{MWe}$ SMR to be $\$ 8.53 / \mathrm{MWh}$ and $\$ 12.05-\$ 25.49 / \mathrm{MWh}$, respectively, depending on whether the unit is a FOAK or NOAK unit (Rosner and Goldberg 2011). For this economic study on water-cooled SMRs, an O\&M cost of $\$ 18 / \mathrm{MWh}$ was deemed reasonable. O\&M and fuel costs may differ substantially for non-water-cooled SMRs. The O\&M costs for the HTR-PM reactor are \$7.6/MWh (IAEA 2006).

Fuel costs alone may also be estimated by looking at both the front and back ends of the fuel cycle. The Nuclear Energy Agency estimates that the front end of the cycle from mining to fuel fabrication costs approximately $\$ 7 / \mathrm{MWh}$, and the back end for both closed and once-through cycles costs $\$ 2.33 / \mathrm{MWh}$ (NEA and OECD 2011). This makes the total fuel cycle cost \$9.33/MWh. The Nuclear Energy Agency's global mean fuel costs are $\$ 9.10 / \mathrm{MWh}$ (IEA 2010). This mean fuel value was used for modeling the water-cooled SMRs. The cost per MWh for large reactors was considered reasonable, as economies of scale do not apply to fuel costs, which follow a constant scale (Rothwell 2007). It is possible that fuel fabrication or other specific aspects of the fuel cycle may see learning effects as one progresses from FOAK to NOAK units. The fuel cost for the HTR-PM design was \$10.90/MWh (IAEA 2006; NEA and OECD 2011).

Decommissioning costs play a minimal role for reactors, as they are spread over the entire 60 -year lifetime of the plant. The Nuclear Energy Agency approximates the cost of decommissioning for large reactors as $\$ 0.16 / \mathrm{MWh}$ for a $5 \%$ discount rate or $\$ 0.01 / \mathrm{MWh}$ for a $10 \%$ discount rate (IEA 2010). The decommissioning costs are assumed to be included in the back end of the fuel cycle and are not treated separately in this economic study. 


\subsection{Summary of Input Data}

Table 5 summarizes the data that may be used for preliminary cost estimates for water-cooled SMRs. It also includes whether the large reactor used for scaling has the capacity for cogeneration. The other factors required may be taken from Table 4.

Table 5. Summary of SMR data required for economic model.

\begin{tabular}{|l|l|}
\hline & \multicolumn{1}{|c|}{ Water-Cooled SMRs } \\
\hline LR Unit Power & $1260 \mathrm{MWe}^{\mathrm{a}}$ \\
\hline Capacity Factor & $90 \%$ \\
\hline Lifetime & 60 years \\
\hline Specific Capital Cost & $\$ 3767 / \mathrm{kWe}$ \\
\hline O\&M Cost & $\$ 18 / \mathrm{MWh}$ \\
\hline Fuel Cost & $\$ 9.10 / \mathrm{MWh}$ \\
\hline Max Outlet Temp. & $\sim 300^{\circ} \mathrm{C}$ \\
\hline Cogeneration & Low Temp. Process Heat \\
\hline
\end{tabular}

a. $1260 \mathrm{MWe}$ capital cost was scaled from the actual $1350 \mathrm{MWe}$ and adjusted to 2014 dollars ( $2.5 \%$ inflation rate).

b. Scaled from the reported specific capital cost for the 1350 MWe ABWR and adjusted to 2014 dollars, assuming a $2.5 \%$ inflation rate. ${ }^{2}$

c. $\quad 10-40 \%$ reduction in O\&M and Fuel Costs for NOAK.

\section{ECONOMIC ANALYSIS RESULTS}

The economic model presented attempts to portray the cost reductions that occur as an SMR technology progresses to maturity (i.e., from FOAK to NOAK costs). Figure 9 demonstrates the specific capital costs relative to the 1260 MWe reactor costs for both FOAK and NOAK SMRs based upon the rated power.

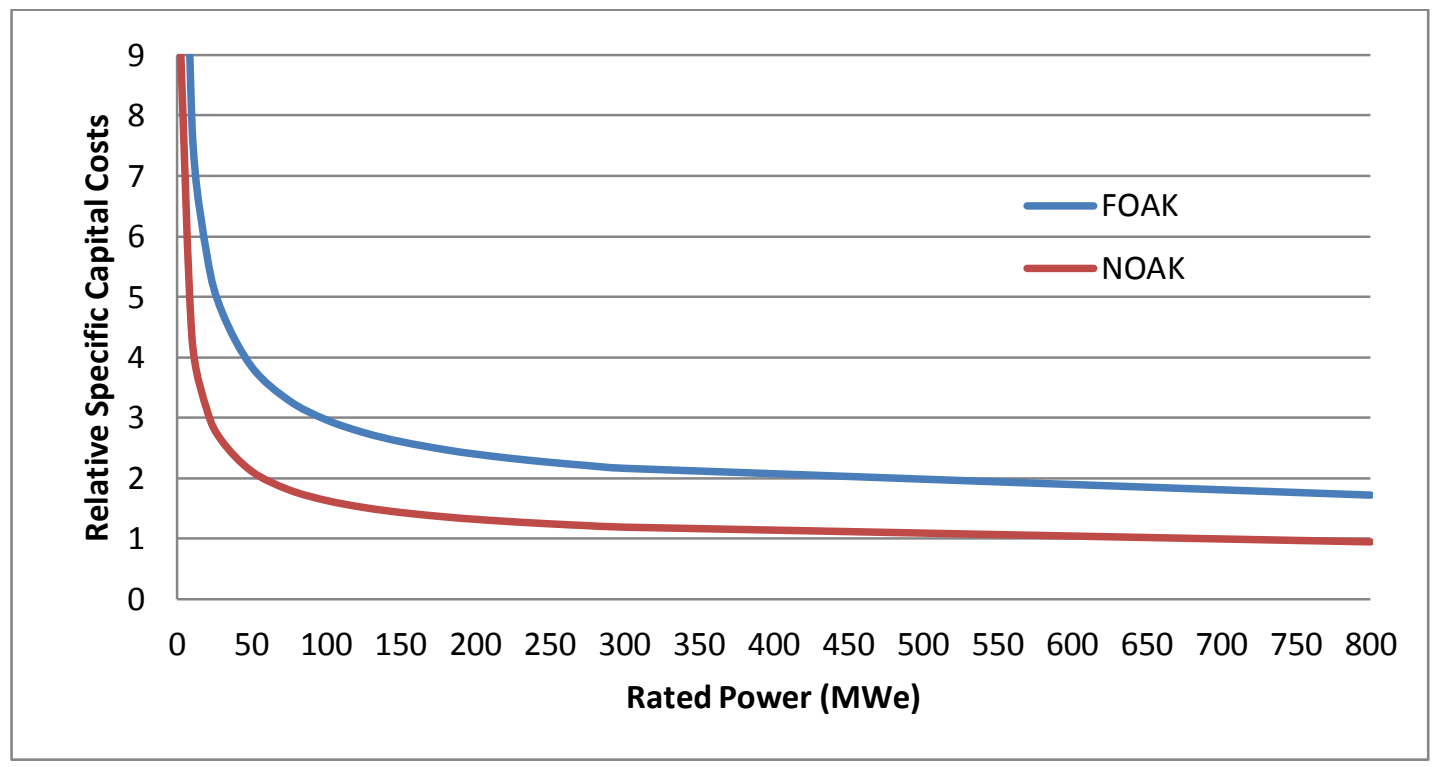

Figure 9. Relative specific capital costs FOAK and NOAK.

\subsection{TCIC Calculations}

Table 6 provides a summary of the calculations and resulting capital costs for FOAK and NOAK SMRs, demonstrating how the capital cost may be greatly reduced via the factors previously described. The costs may be further reduced by sharing of components, systems, and facilities between twin and multi-module plant configurations. The ranges provided represent the learning ranges associated with 
plant configuration. Low FOAK or NOAK costs represent the least reduction in plant configuration learning, while high costs represent the maximum reduction due to plant configuration learning. Overall, with a $4.5 \%$ technological learning rate, the transition from FOAK to NOAK represents a nearly $50 \%$ reduction in TCIC. A major component in these reductions is the perceived risk involved with new technologies. As a result, FOAK SMRs may have additional contingency costs of up to $55 \% .{ }^{2}$ Appendix $\mathrm{G}$ contains the Excel outputs.

Table 6. FOAK and NOAK TCIC.

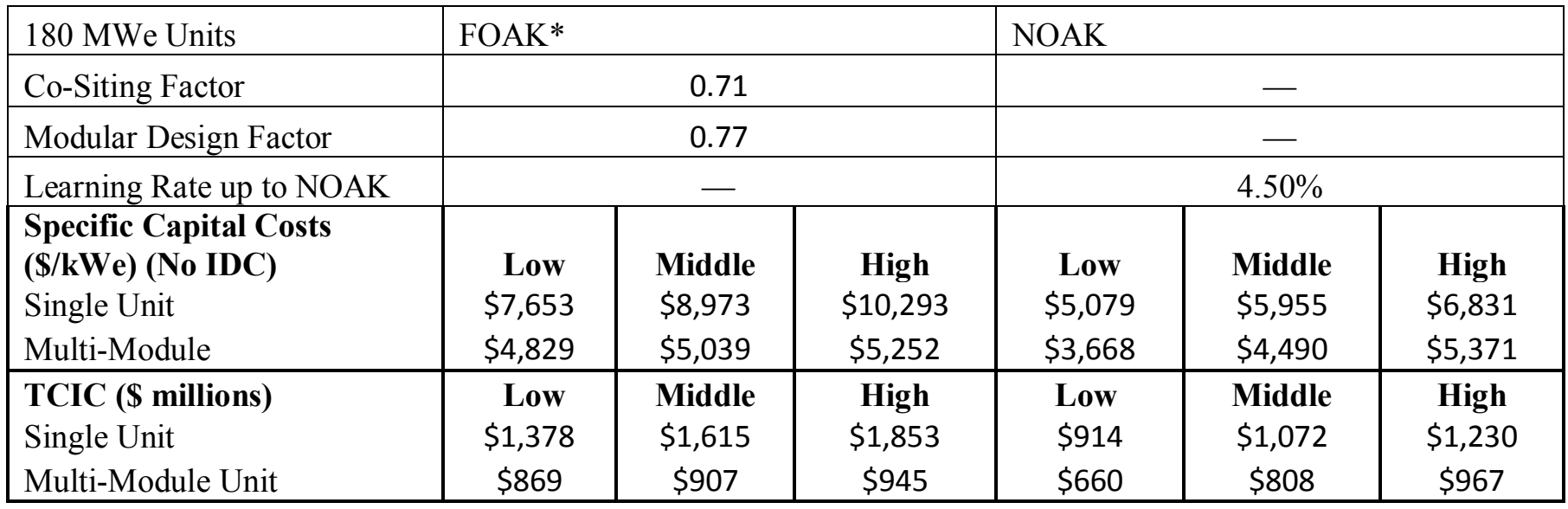

* DD\&E costs were divided over all units up to but not including the first NOAK unit.

\subsection{NPV, LCOE, and IRR}

A major advantage to constructing multiple SMRs on a site rather than one large reactor is that the capital for all units is not required upfront. A strategic plan in which each additional unit is phased in while the others are already operating reduces the upfront capital required. Table 7 shows three different SMR construction scenarios to compare the effects on NPV, IRR, and annual cash flows. It should be noted that for all calculations, the large reactor construction was assumed to take 6 years, while the SMR construction was assumed to take 3 years. Additionally, the TCIC costs were divided over the construction period. DD\&E costs were divided over all FOAK units. Figure 10 shows the cumulative cash flows for seven 180 MWe units following the deployments in Table 7. The middle values from Table 6 were used for the TCIC.

Table 7. Example SMR construction deployment schedules.

\begin{tabular}{|l|l|l|l|l|l|l|l|l|l|l|l|l|l|}
\hline Year & 1 & 2 & 3 & 4 & 5 & 6 & 7 & 8 & 9 & 10 & 11 & 12 & 13 \\
\hline LR & & & & & & & & & & & & & \\
\hline Deployment 1 & & & & & & & & & & & & & \\
\hline 0 yrs. between units & & & & & & & & & & & & & \\
\hline & & & & & & & & & & $\ldots \ldots$ & & & \\
\hline Deployment 2 & & & & & & & & & & & & & \\
\hline 1 yr. between units & & & & & & & & & & & & & \\
\hline & & & & & & & & & & & & $\ldots .$. & \\
\hline Deployment 3 & & & & & & & & & & & & & \\
\hline 2 yrs. between units & & & & & & & & & & & & & \\
\hline
\end{tabular}




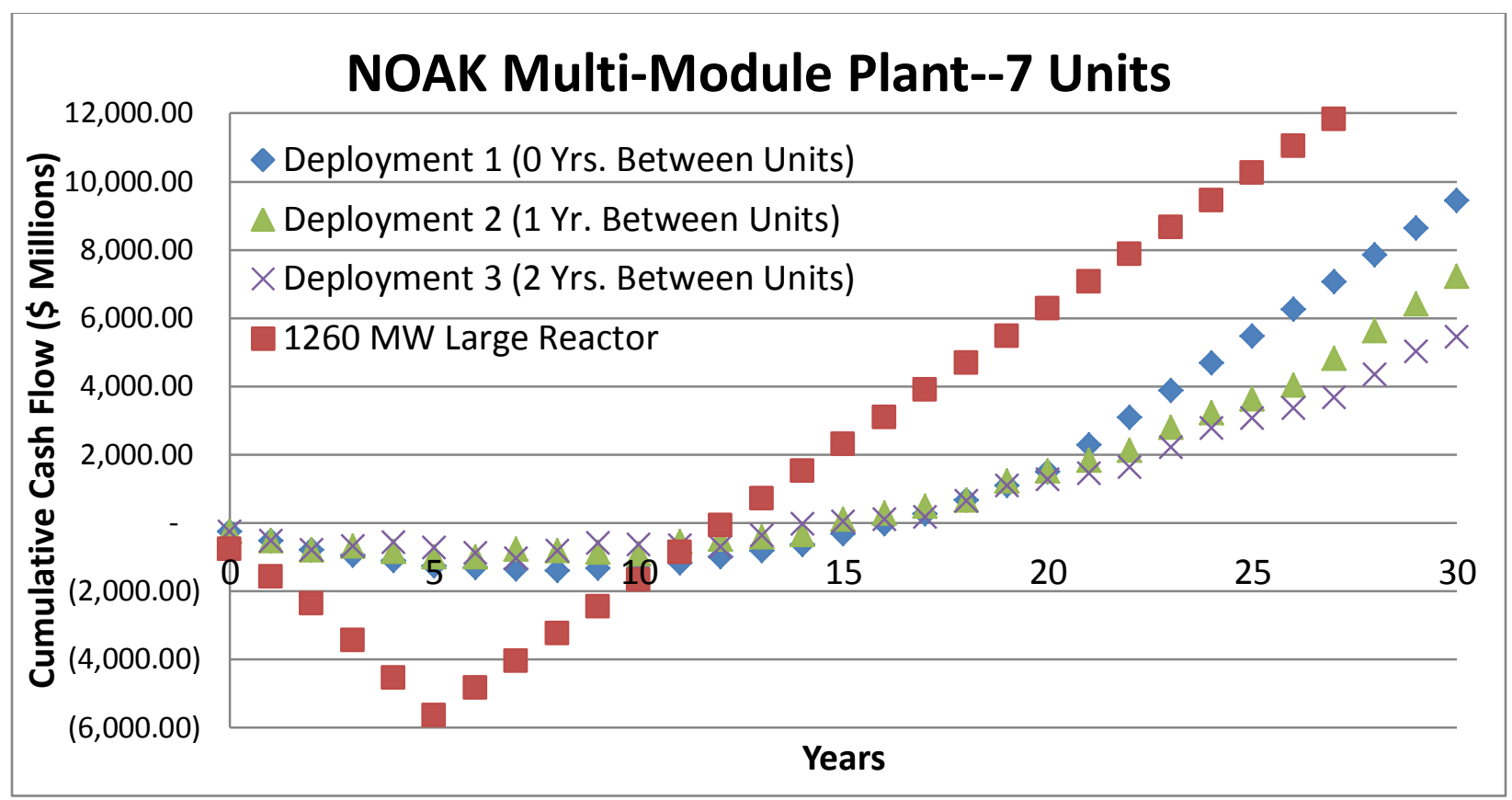

Figure 10. Cumulative cash flows for a seven-unit 180-MWe multi-module NOAK plant with the deployment schedules shown in Table 7 and a 1260 MWe LR.

The reported WACC for this SMR study was calculated as $10 \%$, with a 50-50\% split between debt and equity, a corporate tax rate of $35 \%$, a $10 \%$ interest rate or cost of debt, and a $10 \%$ cost of equity (Putnum Investments 2014). Table 8 displays the NPV, IRR, and LCOE for each SMR deployment and for the 1260 MWe reactor. The real discount rate of $7.32 \%$ calculated from Equation 13 was used for the NPV calculations. The IRR depends on the electricity price assumed. For a project to be economically viable for a firm, the IRR must be greater than the WACC. To accommodate this requirement, the electricity cost was altered until this became true.

Table 8. NPV, IRR, required electricity price, payback, and LCOE for seven-unit 180-MWe multi-module NOAK plant.

\begin{tabular}{|l|l|l|l|l|}
\hline & NPV (\$ Millions) & $\begin{array}{l}\text { Assumed } \\
\text { Electricity Price }\end{array}$ & LCOE & Payback \\
\hline $\begin{array}{l}\text { SMR } \\
\text { Deployment 1 }\end{array}$ & $\$ 1,796-\$ 2,874$ & $11 \mathrm{c} / \mathrm{kWh}$ & $\$ 67-\$ 84 / \mathrm{MWh}$ & 18 years \\
\hline $\begin{array}{l}\text { SMR } \\
\text { Deployment 2 }\end{array}$ & $\$ 1,585-\$ 2,587$ & $11 \mathrm{c} / \mathrm{kWh}$ & $\$ 66-\$ 83 / \mathrm{MWh}$ & 16 years \\
\hline $\begin{array}{l}\text { SMR } \\
\text { Deployment 3 }\end{array}$ & $\$ 1,303-\$ 2,184$ & $11 \mathrm{c} / \mathrm{kWh}$ & $\$ 67-\$ 84 / \mathrm{MWh}$ & 16 years \\
\hline LR 1260 MWe & $\$ 1,976$ & $11 \mathrm{c} / \mathrm{kWh}$ & $\$ 89 / \mathrm{MWh}$ & 14 years \\
\hline
\end{tabular}

The IRRs of the SMRs and the LR should be approximately the same if the total electricity generation is comparable (Carelli 2008). In this case, the IRRs area all set to the same value by changing the electricity prices. The LCOE values are also very similar for the SMRs and LRs. The primary differences occur in the NPV and payback periods. 


\subsection{Sensitivity}

The cost of equity and debt greatly affect the LCOE and NPV of a nuclear project. As the share of debt increases, the cost of equity also increases, and the rate of the increase is related to the cost of debt or interest rate. Figure 11 demonstrates this relationship.

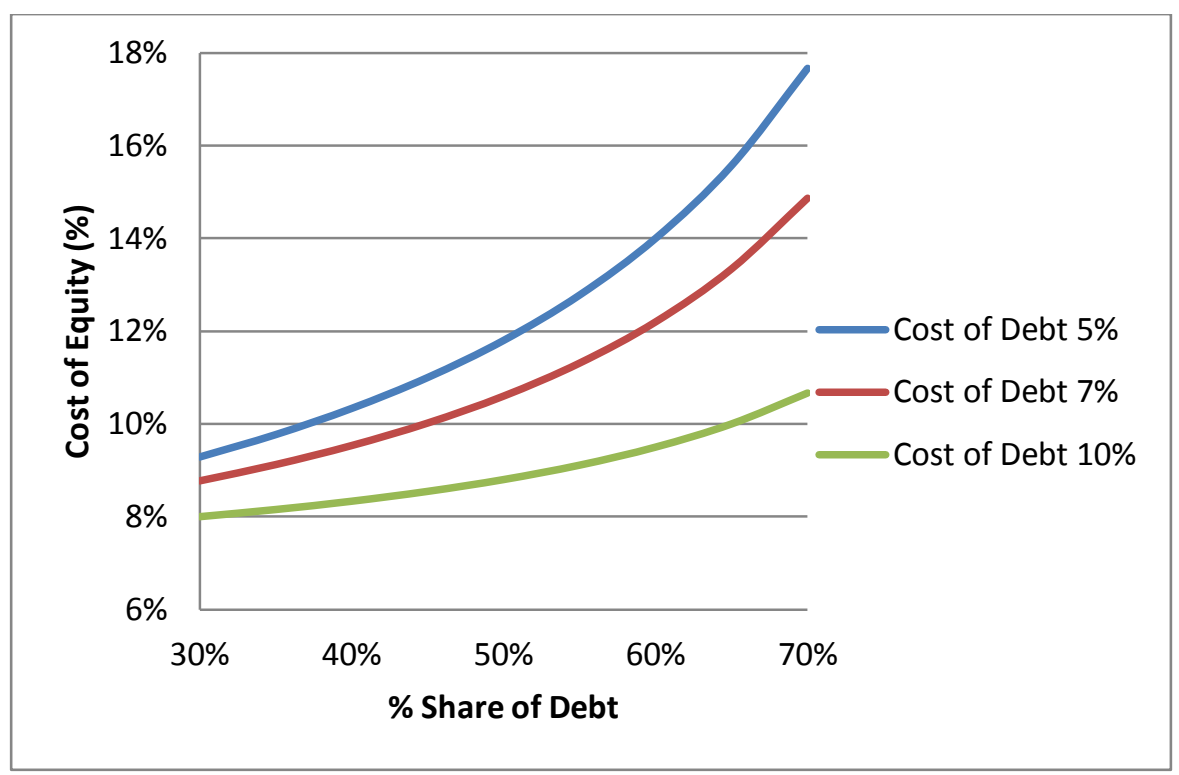

Figure 11. Cost of equity at different debt shares.

The LCOE and NPV are sensitive to changes in market factors, such as equity and debt shares and interest rates. Figure 12 shows the LCOE at different debt and equity shares at two different cost of equity values $-10 \%$ and $15 \%$. Figure 13 shows the sensitivity of NPV to these factors. The IRR, on the other hand, is sensitive to changes in electricity prices. The IRR, on the other hand, is sensitive to changes in electricity prices, as illustrated in Figure 14. Figures 12-14 represent LCOE, IRR, or NPV values for the SMR Deployment 1 with an assumed electricity price of $10.1 \notin / \mathrm{kWh}$. 


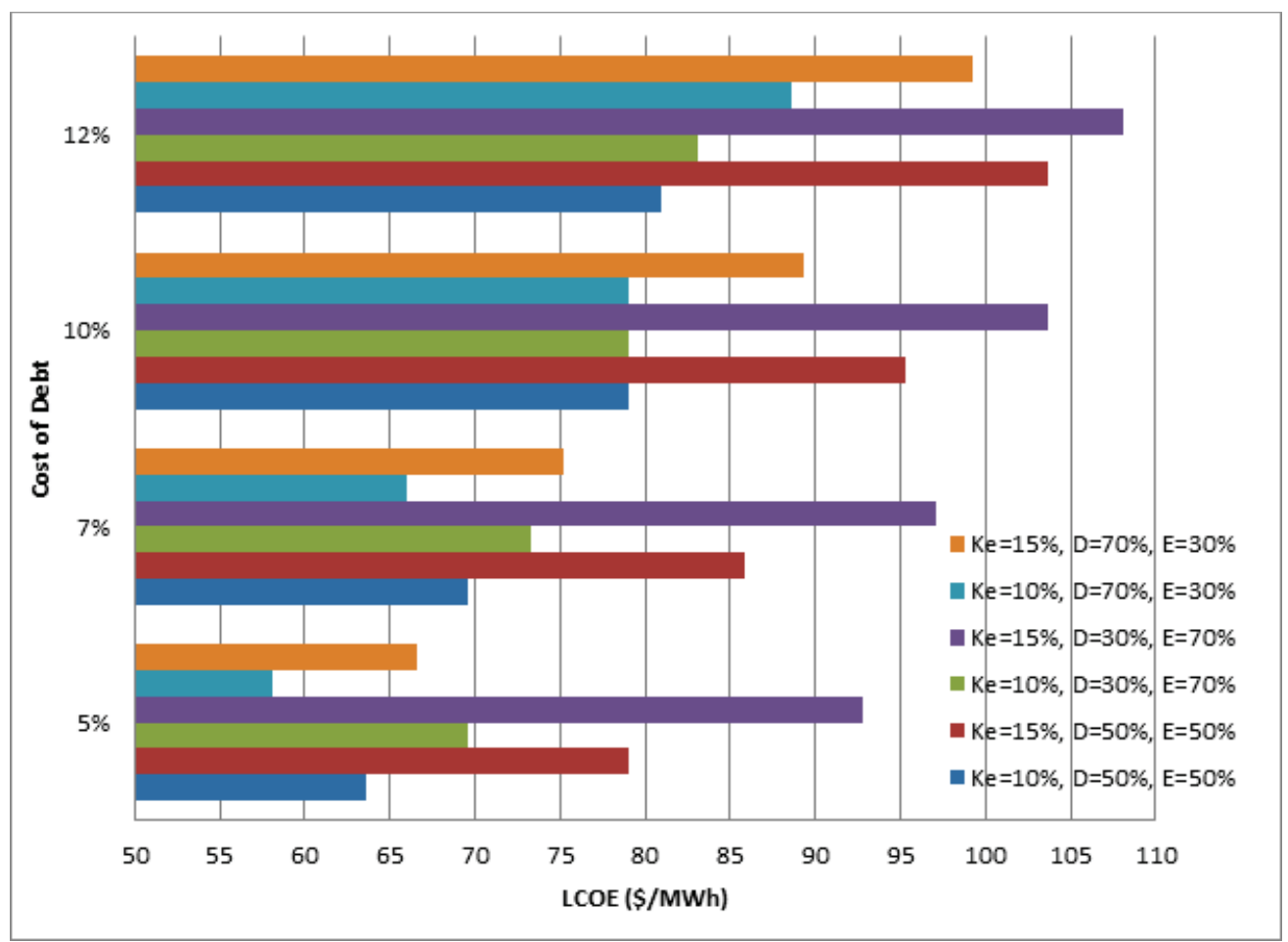

Figure 12. LCOE sensitivity to debt and equity shares, cost of equity, and cost of debt.

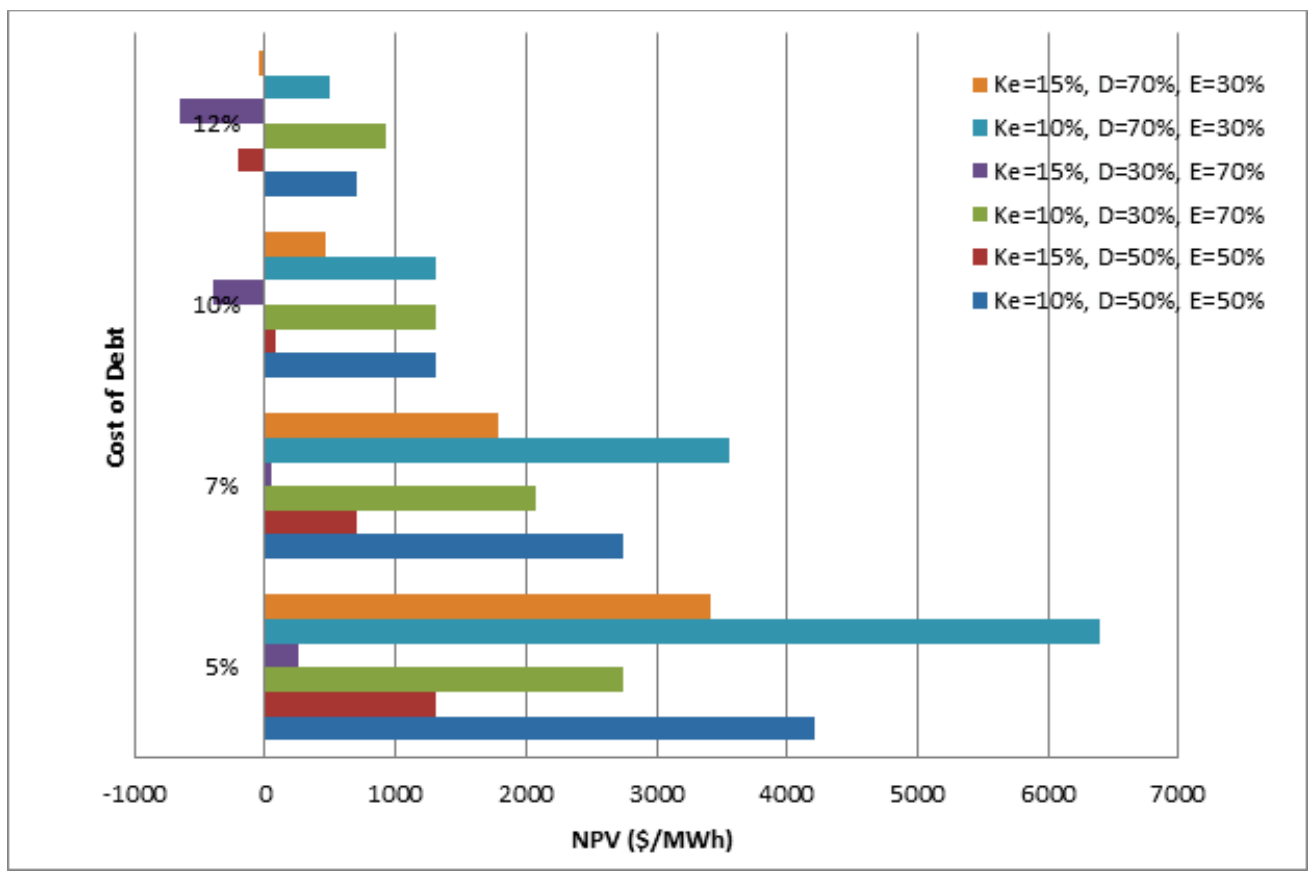

Figure 13. NPV sensitivity to debt and equity shares, cost of equity, and cost of debt. 


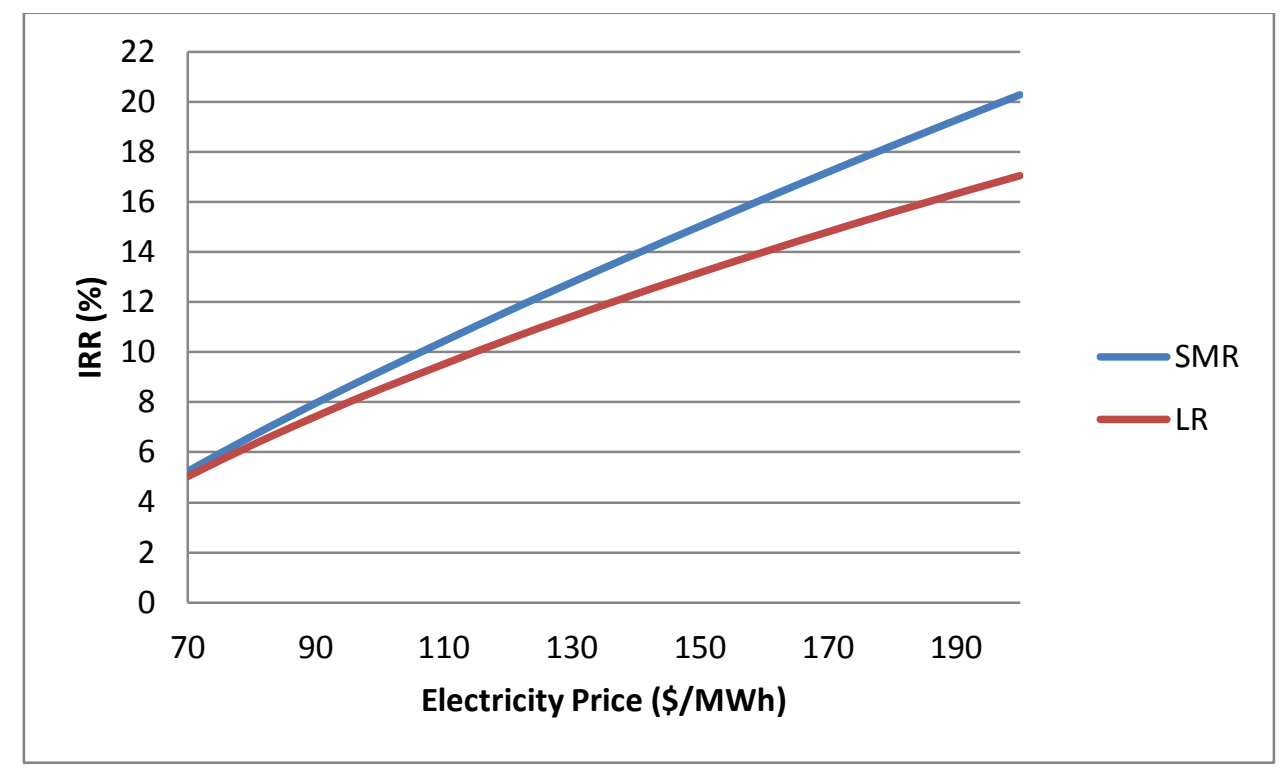

Figure 14. IRR sensitivity to electricity price.

\subsection{Case Studies}

Some example case studies were performed to see if SMRs could feasibly meet the demands of a particular community. Appendix I contains a case study for installing an SMR in Bethel, Alaska, one of many small Alaskan villages with very high electricity rates. It also contains the case study for Anchorage, Alaska in which six 100-MWe SMRs were installed on one site. Alaska was of particular interest due to its high electricity rates. Other regions could also be analyzed further. Hawaii, for example, is another state that has unusually high electricity prices ranging from $\$ 0.30-\$ 0.45 / \mathrm{kWh}$ with electricity load demand much higher than the small Alaskan villages (EIA 2014). Furthermore, Hawaii is trying to reduce its dependence on fossil fuels by setting clear energy goals of $70 \%$ by 2030 . This goal seeks to have $40 \%$ of electricity generated by renewable resources with an additional $30 \%$ provided by efficiency improvements (Hawai'I Powered Clearn Energy Initiative 2010). This would be a ripe opportunity for SMRs to enter in the market. The higher upfront costs associated with FOAK facilities would be mitigated by the already high electricity prices. Additionally, Hawaii seeks to add renewable resources, but has limited land availability for both wind and solar technologies. Alternatively, nuclear power produces clean air energy and requires limited lands.

\section{NON-ELECTRIC APPLICATIONS}

Non-electric applications are often coupled with electricity production to (a) mitigate the high specific capital costs, (b) improve efficiency, and (c) help stabilize electric output instabilities due to intermittent renewable energies. SMRs could provide electricity and/or heat for applications such as desalination, district heating, and industrial process heat. However, because competition is the primary driver for such systems, SMRs must compete directly with fossil fuel heat/electricity production and will depend upon specific location costs and future factors such as fuel price and construction times (IAEA 2007).

\subsection{Combined Heat and Power}

"Combined heat and power (CHP) can be an efficient and clean method of generating electric power and useful thermal energy from a single fuel source at the point of use. Instead of purchasing electricity from the local utility and burning fuel in an on-site furnace or boiler to produce needed thermal energy, an industrial or commercial user can use CHP to provide both energy services in one energy-efficient step. Consequently, CHP can provide significant energy 
efficiency and environmental advantages over separate heat and power. As with all power generation, CHP deployment has unique cost, operation, and other characteristics, but it is a proven and effective available clean energy option that can help the United States enhance energy efficiency, reduce greenhouse gas $(G H G)$ emissions, promote economic growth, and maintain a robust energy infrastructure"'(State and Local Energy Efficiency Action Network 2013).

Presently, there are more than $4000 \mathrm{CHP}$ facilities in the U.S., producing $82 \mathrm{GW}$, and according to McKinsey and Company, an additional $50 \mathrm{GW}$ could be produced at other sites with acceptable rates of return (State and Local Energy Efficiency Action Network 2013; McKinsey 2009). As previously mentioned, CHP facilities may drastically improve energy production efficiencies and typically have efficiencies of 60-80\%, whereas as separate electricity and heat systems tend to have efficiencies of $45-$ $50 \%$ (State and Local Energy Efficiency Action Network 2013). This efficiency increase is due to the use of recovered heat for heating/cooling, as shown in Figure 15 (EPA Combined Heat and Power Partnership 2013).

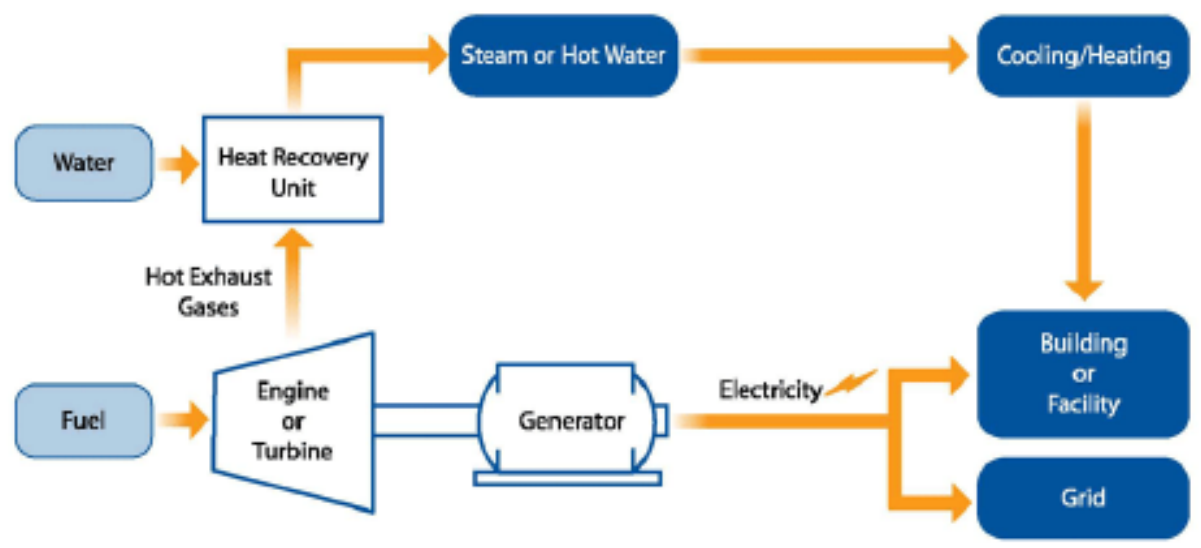

Figure 15. Example CHP facility (EPA Combined Heat and Power Partnership 2013).

CHP systems may also be designed to meet specific heat and electricity needs. Figure 16 demonstrates how the required inputs are significantly less than conventional power generation. In this particular example, the overall efficiency is improved by $24 \%$. 


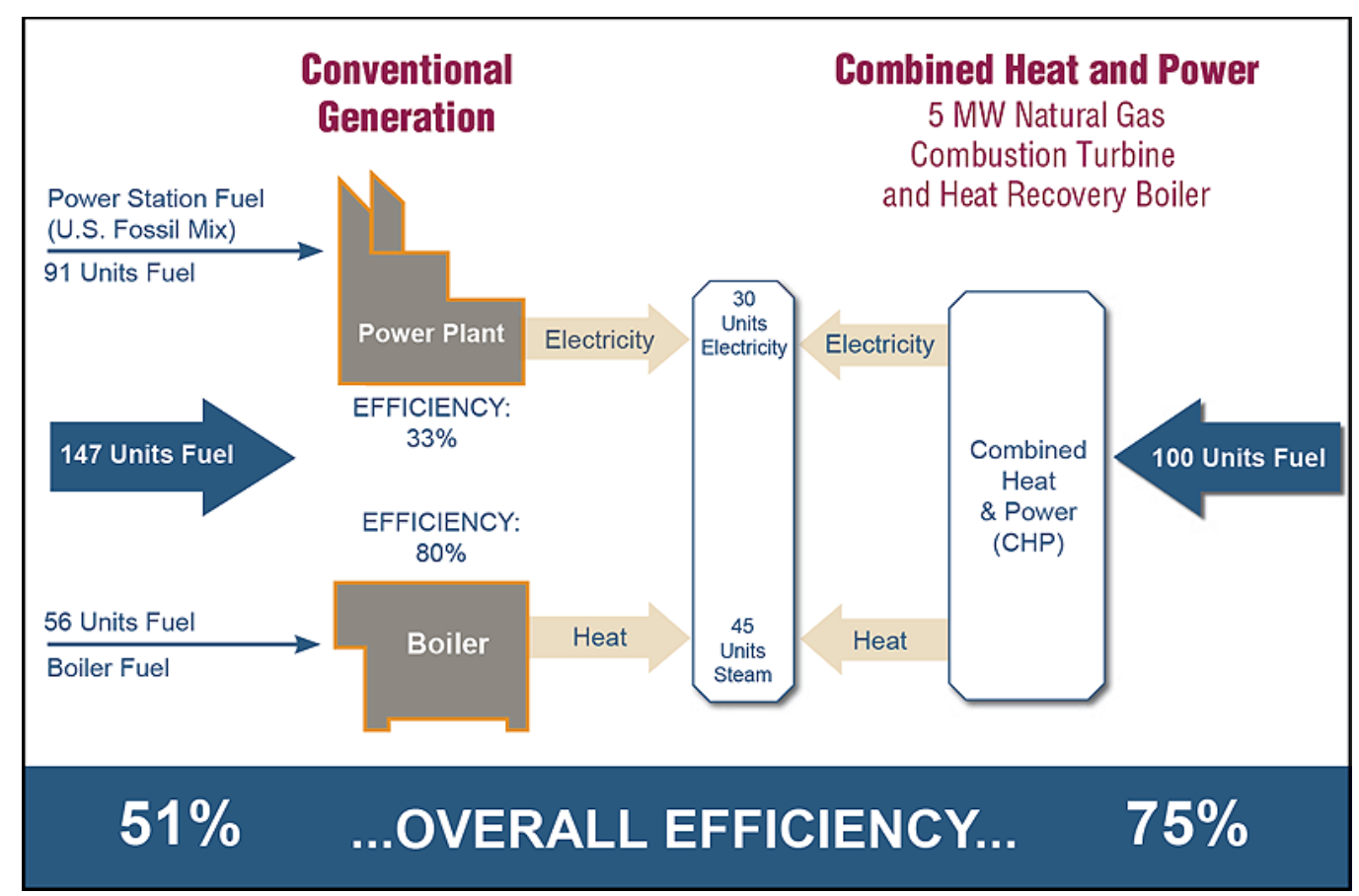

Figure 16. Example CHP Facility (EPA Combined Heat and Power Partnership 2013).

\subsection{Desalination}

Many countries have regions that utilize or could benefit from desalination processes. However, many of them do not have any nuclear generating capabilities; therefore, they are lacking the infrastructure, regulatory oversight, financing, and fuel management capabilities required to incorporate a nuclear reactor as the power or heat source for a desalination plant (IAEA 2007). On the other hand, there are several countries with nuclear that have regions lacking water, such as China, Korea, India, and Pakistan. Combined heat and power plants would be useful within these regions, yet they must compete, as previously mentioned. Higher fossil fuel prices would support the use of nuclear CHP plants, whereas higher interest rates would support fossil fuel development for desalination plants (IAEA 2007).

\subsection{District Heating}

District heating is another CHP application, in which the heat supplied goes directly to heating homes and water in locations with particularly cold or long winters. This type of system requires infrastructure to transport the heat and the CHP facility must be nearby the area being heated to prevent significant losses. As a result, a nuclear facility would have to demonstrate enhanced safety system and a low core damage frequency (IAEA 2007). This is where SMRs would be better suited than a large nuclear reactor. Furthermore, SMRs would help with pollution problems in more heavily populated cities. The major downsides are the seasonal load requirements, typically less than $50 \%$, and the competition with electric heating (IAEA 2007).

\subsection{Industrial Process Heat}

Many industrial applications require high reliability and must have power and/or heat facilities nearby capable of operating at high temperatures with load factors of 70-90\% (IAEA 2007). CHP plants are often utilized to meet these needs. SMRs of different designs are well suited for different CHP industrial processes, as shown in Figure 3. Oil sand open-pit mining and deep sea extraction are two processes that HTG-SMRs would be particularly well-suited with outlet temperatures between 300 and $600^{\circ} \mathrm{C}$ (IAEA 2007). Another important industrial process is hydrogen production through electrolysis (NEA and OECD 
2011). Excess SMR heat used to produce hydrogen may provide an additional revenue stream and help lower the overall facility costs.

\section{NUCLEAR HYBRID ENERGY SYSTEMS (NHES) UTILIZING SMRS 10.1 Electricity Demand}

Power demand is met through the use of base, intermediate, and peak load. Base-load power is provided by consistent energy production methods, which are often unable to fluctuate with hourly demand changes. These typically include coal and nuclear power, but may also include geothermal and hydropower where available. Base-load power levels are about $35-40 \%$ of the maximum load during the year. An intermediate demand load is one that operates $30-60 \%$ of the time helping to reach more closely to the higher demand levels. This category includes wind, solar, and other renewable methods. Finally, peak demand is met by flexible and controlled methods, like combined cycle natural gas or light oil. These plants only operate $10-15 \%$ of the time (NY Affordable Reliable Electricity Alliance 2008). Due to this low capacity factor, low capital cost peak demand methods are desired, which is usually performed with natural gas.

\subsection{Hybrid Energy Systems}

Hybrid energy systems are being observed as a potential application of intermittent energy production methods, such as solar and wind energy, in combination with a more flexible base-load production method, such as nuclear energy. They are also being looked at as a method of providing ancillary services, such as heat for use in outside processes. SMRs, in particular, generate heat/electricity similar to that required for many large chemical or industrial facilities. Continual operation of these SMRs via electricity or cogeneration would provide optimal usage of these resources, while furthering the development of renewable energies.

Renewable energies cause grid instabilities when their share of the market becomes large due to daily fluctuations in wind and sun availability. Once the market share of intermittent wind or solar is greater than $10 \%$, other consistent energy production methods see a large reduction in revenue. For those systems that cannot follow hourly load demands, such as nuclear and coal, the revenue or price of electricity becomes zero or even negative (see Figure 17).

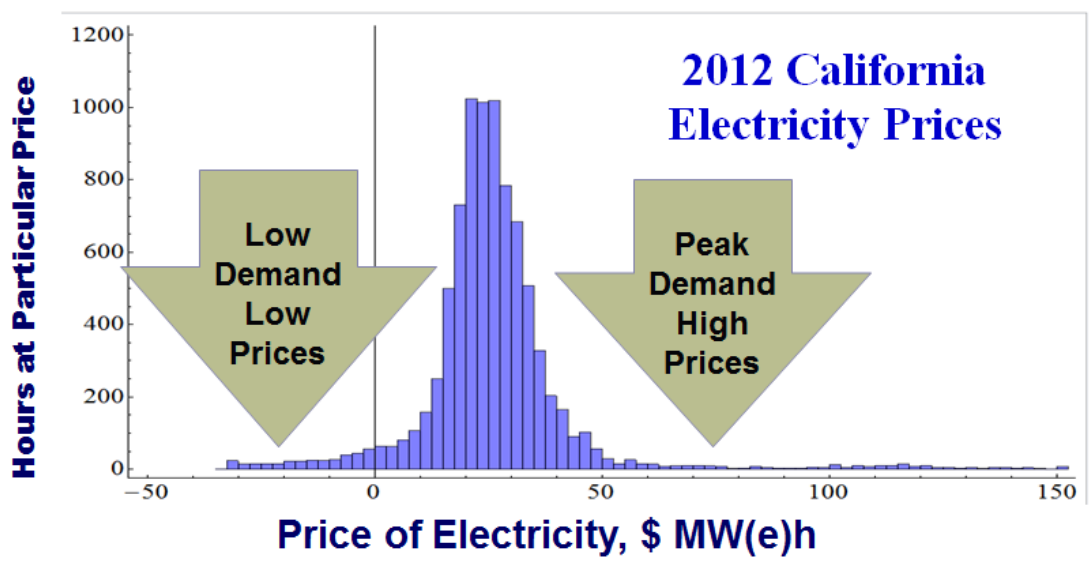

Figure 17. Price of electricity at low and high demand (Forsberg and Schneider 2014).

Unless something is done to strengthen the ability of nuclear plants to respond, utility companies may deem them unprofitable and choose to shorten their lifetime or to operate them at reduced power output. Exelon, for example, may choose to reduce output in the future in its Illinois plants if negative revenue periods are expected to occur in regions where renewables now share a large portion of the electricity generation grid (Daniels 2013). 
A possible solution for this problem is Nuclear Hybrid Energy Storage Systems, in which nuclear heat and/or electricity is stored during period of low revenue due to high wind/solar output and then used during higher revenue periods. This type of system may also be paired with cogeneration processes, such as hydrogen production, desalination, etc. In practice this system would eliminate the periods of low or negative revenue. It would also help slow-to-respond base load systems better match the demand load, which naturally fluctuates on an hourly and seasonal basis. For example, a nuclear plant could operate at some average power level, store excess energy when demand is lower, and use that energy later to meet peak demand.

Figure 18 illustrates a complex example of a nuclear hybrid energy storage system, in which thermal energy is produced from the nuclear reactor steam (Ruth et al. 2014). The steam/heat may either be converted to electricity for the grid or it may be stored by some means. Depending on the temperature required for a particular outside process, the stored heat may be used as is or amplified via temperature boosting to support low, intermediate, and high temperature processes. The type of reactor used will dictate the possible heat output and whether heat boosting is necessary. Example temperature output ranges for different reactors may be found in Figure 4 and Table 2. From storage, the heat may also be converted into electricity and sent back to the grid. This occurs when wind energy production is low or when peak demand must be met.

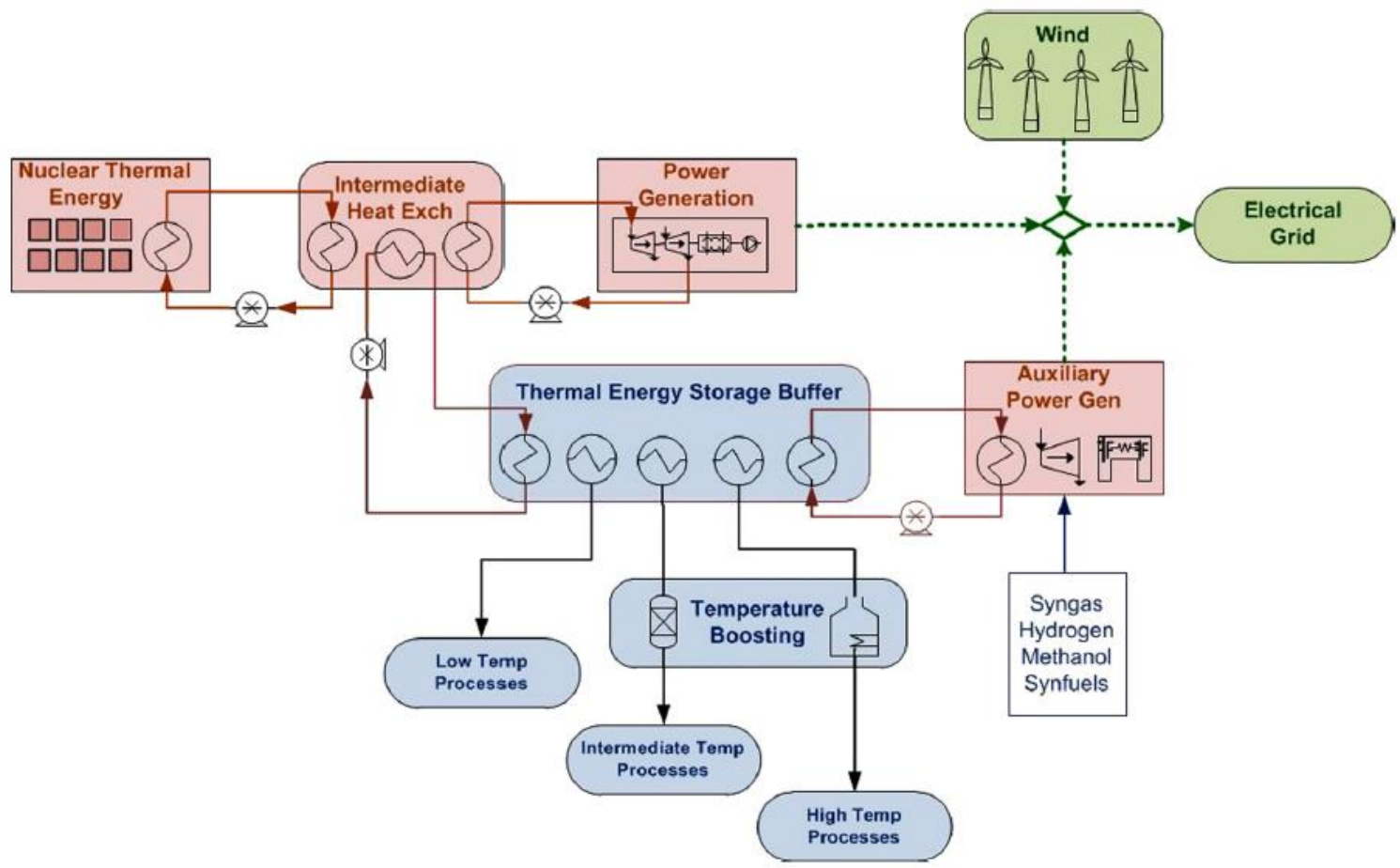

Figure 18. Illustration of a possible nuclear hybrid energy storage system (Ruth et al. 2014).

\subsection{Storage Types and Potential}

Many different types of storage facilities may be used to store heat or electricity from batteries up to pumped hydro. Each facility has limitations in the amount of energy that may be stored, the discharge times, and the associated capital costs with such infrastructure. Figure 19 illustrates the purpose for each storage system type, including power quality, grid support, and bulk power management (Akhhil et al. 2013). For SMR NHES applications, the storage systems for grid support and bulk power management would seem more useful. A lead-acid battery, for example, would provide up to $10 \mathrm{MW}$ of storage with the ability to discharge the energy in minutes, making it useful in responding to a sudden increase in demand load. On the other hand, if winds were very strong over a day due to storms, it may be 
more useful to store the electricity in Compressed Air Storage (CAES) if there is a lot of power to store, or in a NaS battery if there is less than $10 \mathrm{MW}$ to store. Both of these would allow for discharge times of several hours, allowing the heat to be stored until the wind passes and it is needed.

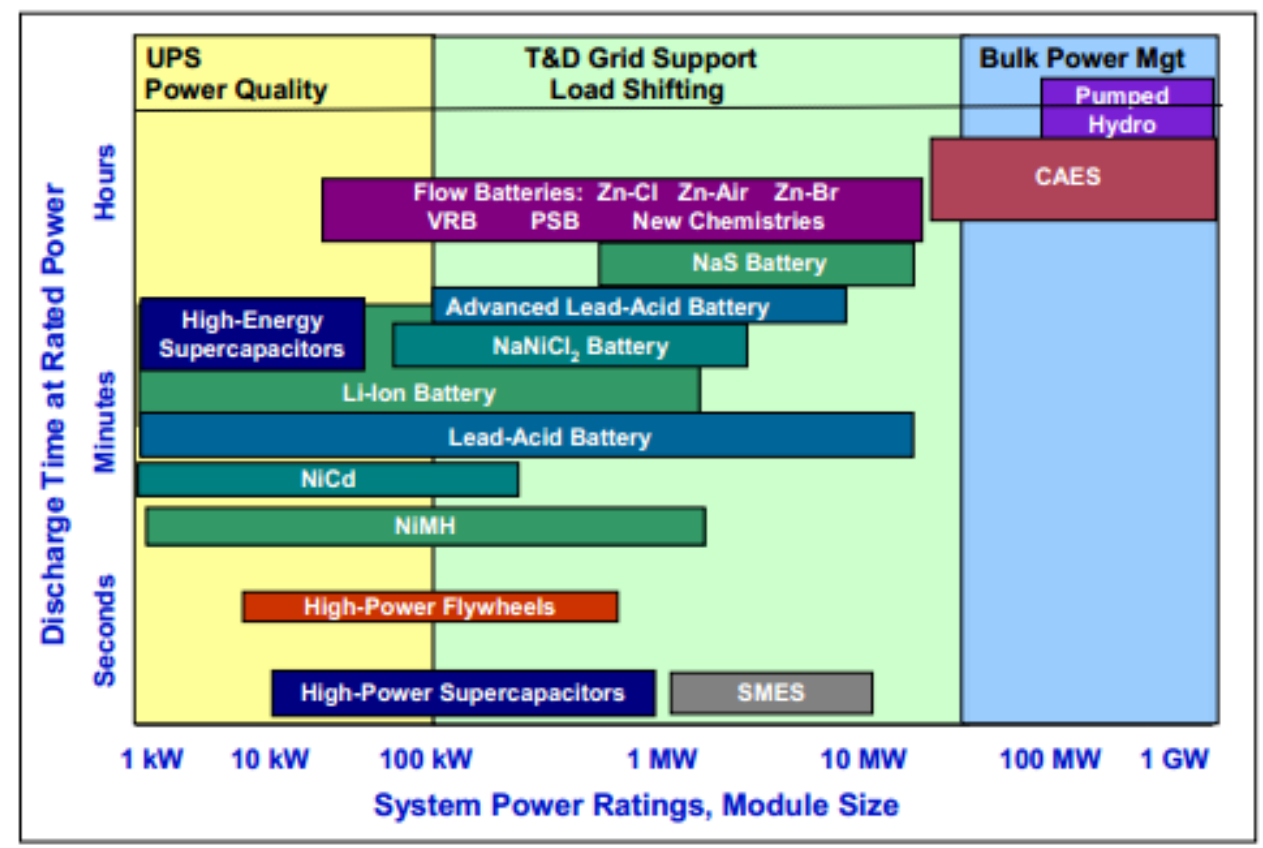

Figure 19. Types of HES and relative discharge times and power ratings (Akhhil et al. 2013).

Pumped hydro is an effective and well-established method of energy storage. Although the discharge time in hours is flexible, typically it is not effective unless more than 8 to 10 hour discharge times are used. This is due to the high costs associated with large reservoirs, dams, and the required engineering work to support them (Akhhil et al. 2013). Pumped hydro is not likely to be used unless significant amounts of energy are stored, as they may store energy up to $1 \mathrm{GW}$. Hydro also requires large bodies of water, which may be limited. A primary advantage of some SMR designs is limited use of water, which facilitates siting in dry areas.

Compressed air energy storage systems also require large costs for caverns or storage reservoirs, particularly if they are placed above ground (Akhhil et al. 2013). Furthermore, they are more suited to higher energy storage greater than $100 \mathrm{MW}$ and require hours for discharge. Other mechanisms, such as flywheels and batteries have much greater flexibility in their deployment and may be better suited to following rapid fluctuations in demand load.

For thermal energy storage alone, there are three general mechanisms: sensible heat storage in which a medium-like molten salt or rocks is heated or cooled, latent heat storage in which phase changes are undergone in phase change materials, and thermo-chemical storage in which energy is stored in chemical reactions (EPA Combined Heat and Power Partnership 2014). Each type of energy storage has different applications to which they are most effective. Sensible heat storage is generally applied on a large scale and is often used to heat water. Its major limitation is the specific heat of the medium used. Latent heat storage relies on specific material phase changes, and as a result, may be used to automatically discharge at a given temperature - for industrial process heat. Thermo-chemical storage systems may have very high storage capacities and offer a variety of applications. Both latent heat and thermo-chemical storage systems are still in the research and development phases, whereas sensible heat storage is commercially available. 
It is important to note that capital costs for these systems will also play a significant role in determining which system is best, and that the specific site for a project will often dictate project feasibility.

\subsection{Prior NHES Market Studies}

Many financial studies have been performed to assess the market benefits of using an NHES system as opposed to a typical base-load system for a particular region. Studies of the ERCOT grid in Texas have demonstrated how coupling the already existing generation plants with storage systems to better follow the demand could drastically improve revenues and help combat the high intermittent energy generation periods which result in low or negative revenues (Tipps et al. 1). In the ERCOT grid, electricity prices are set through competition, as it is a deregulated market. Thus, natural gas fuel prices or wind during peak wind periods will typically drive the electricity prices. Houston, with essentially all power from coal and natural gas, demonstrated prices most heavily affected by natural gas, whereas West Texas with most of its power from wind, coal, and natural gas, saw the effects of both natural gas and wind (Tipps et al. 1). It is due to the wind energy in West Texas that the region could see revenue increases of over $40 \%$ with the addition of storage, whereas Houston could only see increases of less than 20\% (Tipps et al. 1).

Further study into the ERCOT grid demonstrated the tremendous effects that atypical high wind generation could have (Tipps et al. 2). Typically prices for electricity would be set by natural gas or even coal, but with enough wind generation, wind energy will set the prices. Because wind energy has no O\&M and fuel costs, electricity prices drop significantly, and base-load powers such as nuclear energy cannot compete (Tipps et al. 2).

\section{HYBRID ENERGY SYSTEM ECONOMIC ANALYSIS}

\subsection{New York Central Zone C}

New York State is used for this case study, as it is controlled by the New York ISO and the demand follows a predictable daily and seasonal pattern. The demand follows a bell curve with two peaks during the winter and one larger peak during the summer, as shown in Figure 20.

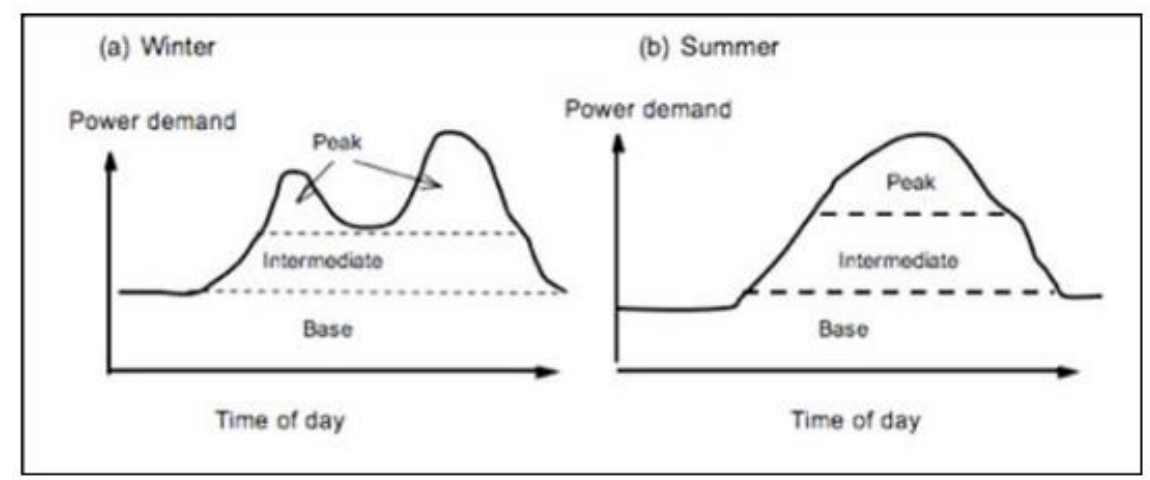

Figure 20. Daily power demand pattern based on season (NY Affordable Reliable Electricity Alliance 2008).

In New York, the base-load power generation is produced by hydropower, nuclear energy, coal, and petroleum. The intermediate and peaking demands are met by natural gas and non-hydro renewables. ${ }^{\text {Error! }}$ eference source not found. Non-hydro renewables account for a small percentage of the state, so natural gas provides a large total percentage of electricity. It is this reliance on natural gas in the New England that causes the electricity prices to follow the cost of natural gas, as demonstrated in Figure 21. In 2010, 45\% of the electricity generated in New England was generated from natural gas (Anjum 2013). 


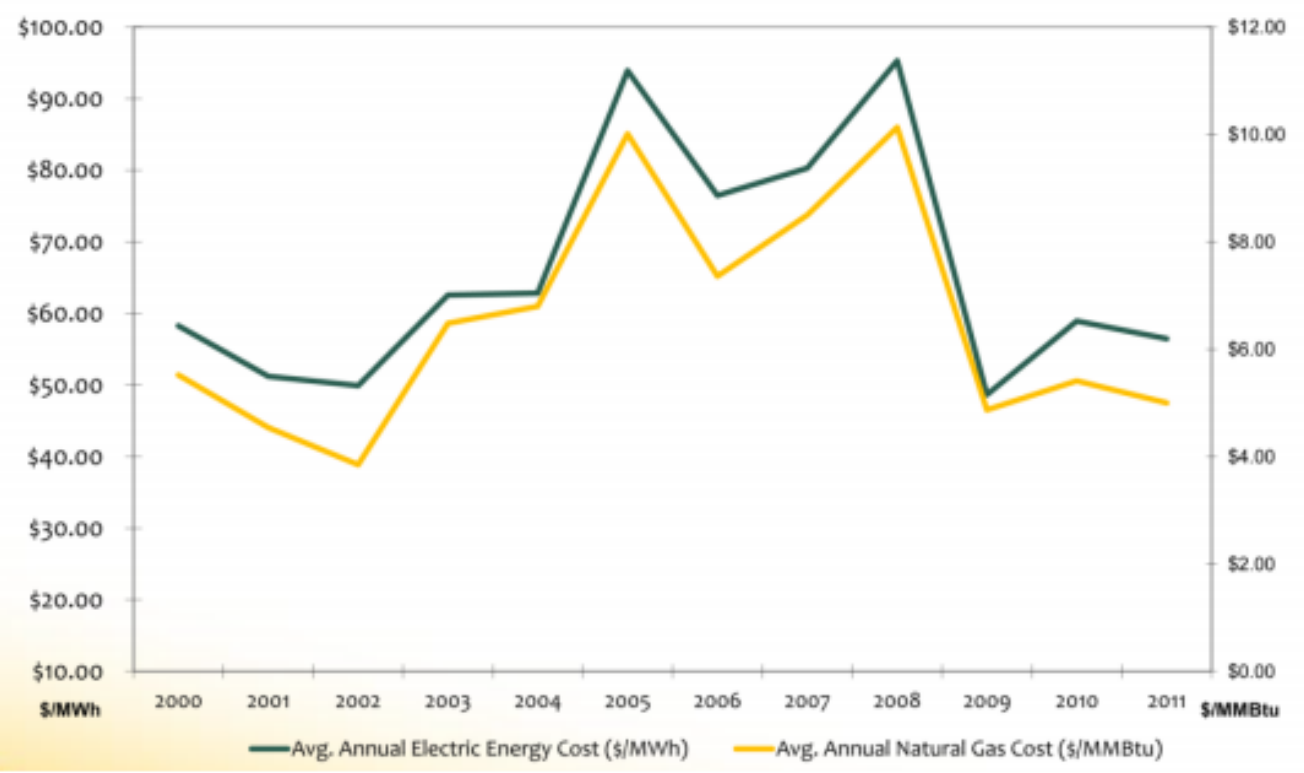

Figure 21. Correlation between natural gas and electricity prices in New England (Anjum 2013).

The NY ISO divides the state into regions. For this case study, Region C in central New York will be analyzed, as it has both nuclear power and wind already established. This would make the region more likely to incorporate NHES in the future. The NY ISO regions are shown in Figure 22.

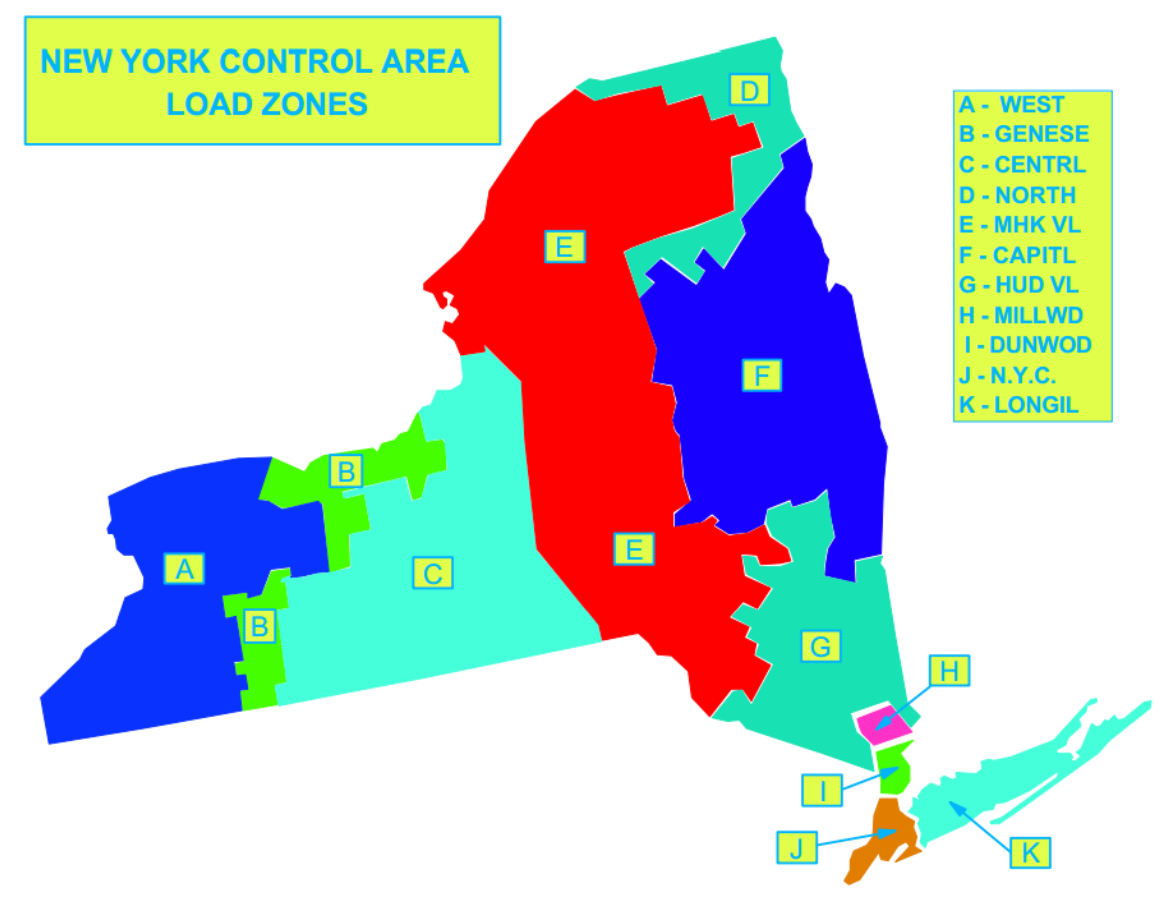

Figure 22. Controlled Load Zones (NY ISO 2014a).

In Region C, base-load power during the summer is provided by coal (379.5 MWe) and nuclear (2760 MWe), while wind power provides part of the intermediate load (418.9 MWe) (NY ISO 2013). The total summer capacity is 6,560.5 MWe (NY ISO 2013). The base-load capacity in this region is nearly $50 \%$ of the total capacity. Although this electricity is generated in this region, much of it is used elsewhere.

Figure 23 shows the demand load for June 25-26 with clear bell curves. The max electricity demand over 
these days is approximately $2350 \mathrm{MWe}$, while the minimum values are approximately $1500 \mathrm{MWe}$. The marginal cost of electricity is also tracked by the NY ISO and is shown in Figure 24 for the same time period. It is clear that the cost of electricity is significantly higher during the peak regions from approximately 11:00 am to 4:00 pm on both June 25 and 26 .

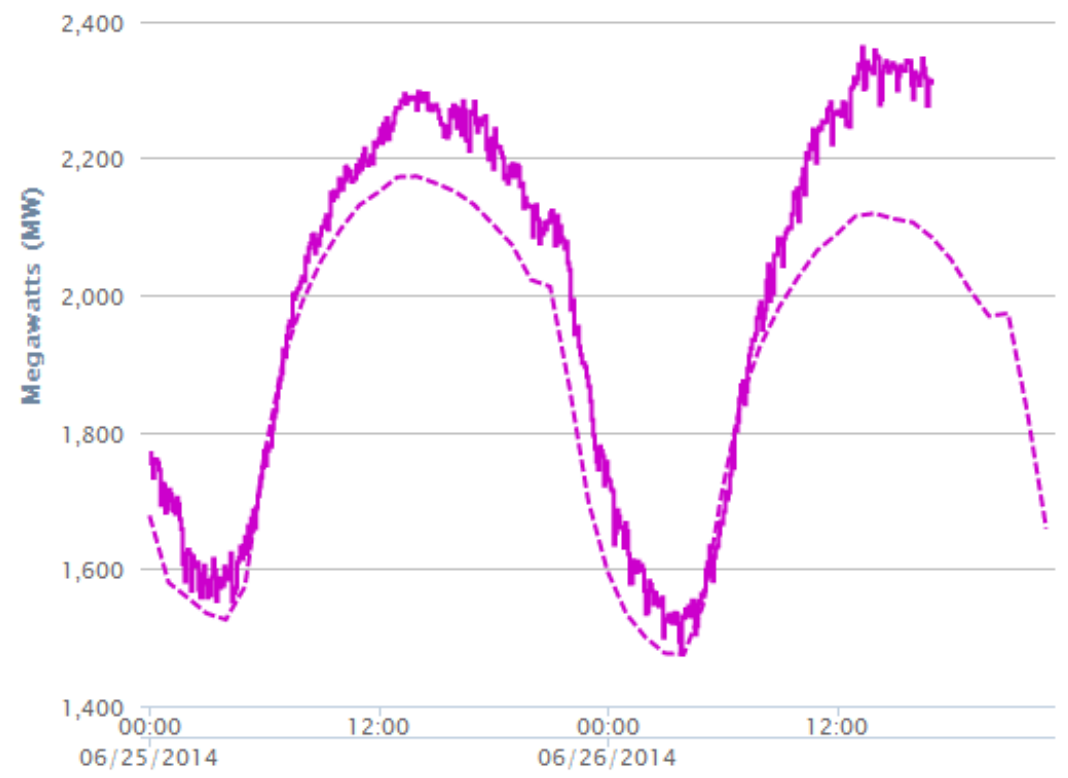

Figure 23. Typical Summer Load in Region C (6/25-6/26): dashed is predicted, regular line is actual (NY ISO 2014b).

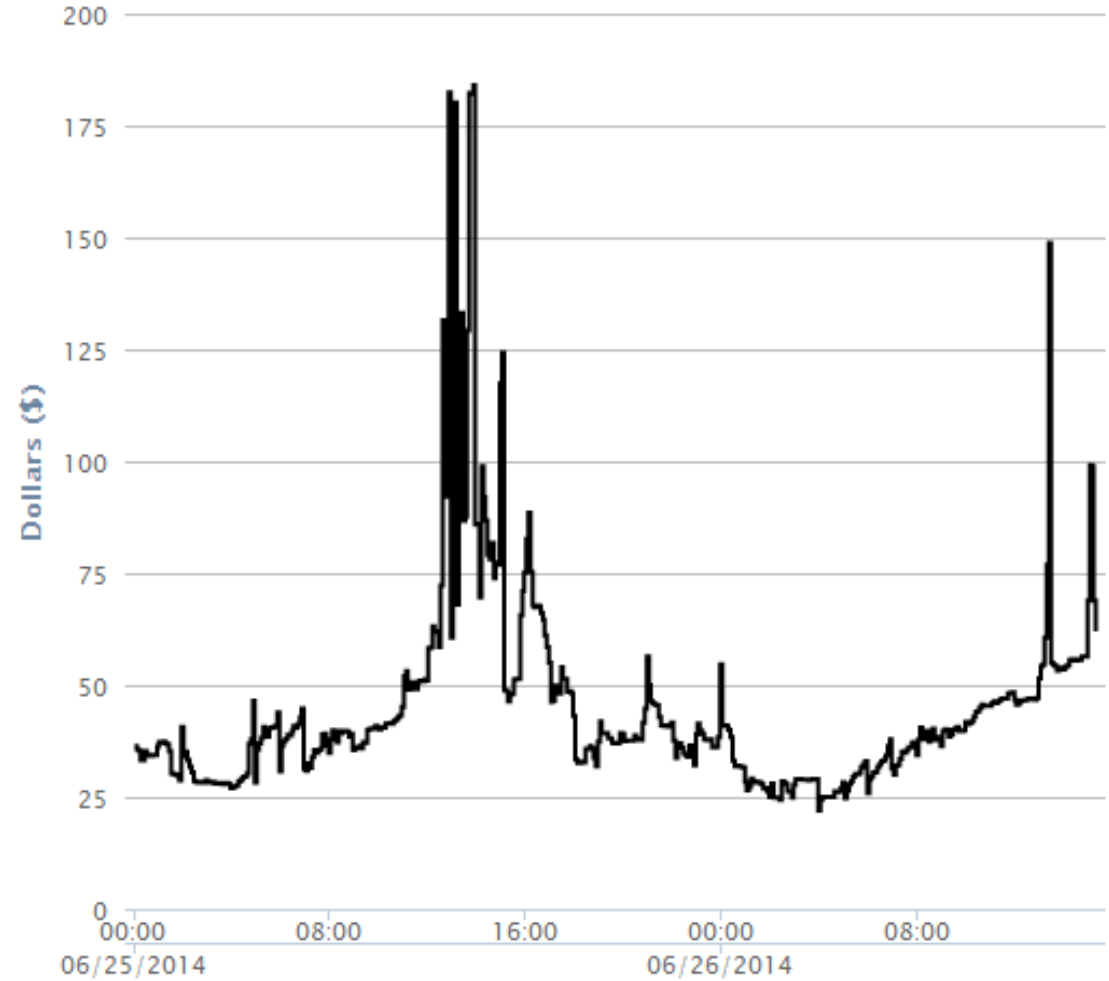

Figure 24. Typical Marginal Cost of Electricity in Region C (6/25-6/26) ${ }^{\text {Error! Reference source not found. }}$ (NY ISO 014b) 


\subsection{NHES Model Inputs}

Three cases were performed, utilizing New York West Central load and wholesale electricity prices for one year, in order to assess the competitiveness of an SMR-wind-natural gas hybrid energy system. The operational costs for the three cases were estimated.

1. Base Case: Electricity generation with no storage and constant wind power at an availability of $30 \%$.

2. Storage Case: Electricity generation with excess nuclear energy stored in compressed air energy storage (CAES) or pumped hydro storage (600 MWe maximum storage). All wind energy available is directed to the electricity grid; nuclear energy is then used to meet the demand, and any excess is stored; natural gas is used to meet demand beyond the wind and nuclear capacities.

3. Hydrogen Production Case: Electricity generation coupled with hydrogen production, designed such that $10 \%$ of the total revenue is from hydrogen production. No storage is present in this case.

An approximation of the base, intermediate, and peak loads for the summer were made and are displayed in Figure 21 monthly load. For the purposes of this economic study, the base load is approximated as $1600 \mathrm{MW}$, while the intermediate load is up to an additional $600 \mathrm{MWe}$, and the peak load is anything above $2200 \mathrm{MW}$.

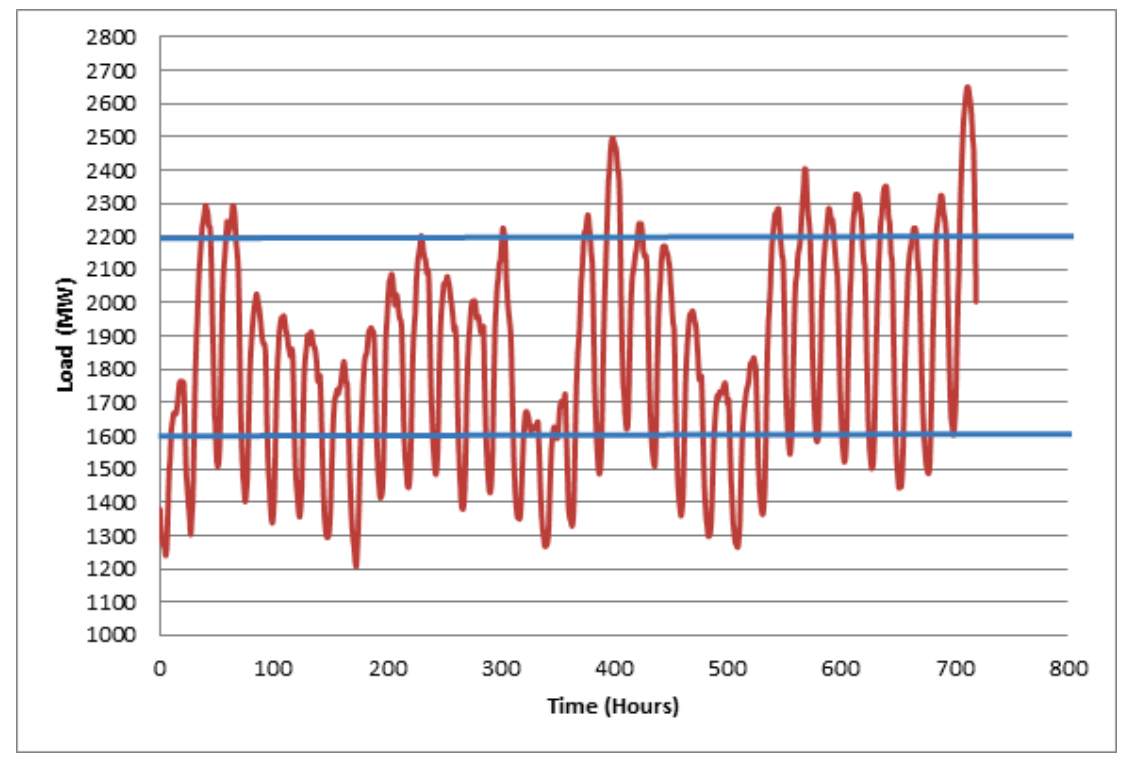

Figure 25. June 2014 summer load (NY ISO 2014b).

The New York West Central region already has electricity generation capacity from both wind and nuclear energy. The maximum nuclear capacity is $2760 \mathrm{MWe}$, coal capacity is $379.5 \mathrm{MWe}$, and the wind capacity is $418.9 \mathrm{MWe}$ (NY ISO 2013). It is assumed that 1639.5 MWe of nuclear capacity and 418.9 MWe wind capacity remain in the area as the base-load and a portion of the intermediate demand. Natural gas/and or storage will meet the remaining intermediate and peak demands required.

The wind energy has $30 \%$ efficiency resulting in a typical production of $125 \mathrm{MWe}$. When the demand is below $1600 \mathrm{MWe}$, the nuclear energy is stored in CAES or pumped hydro storage, which must be able to accommodate up to 600 MWe. CAES and pumped hydro were chosen in this economic study, as they are available commercially and may be used to store large amounts of energy greater than $100 \mathrm{MWe}$. Furthermore, they are well suited with hourly discharge times to help meet the natural daily usage 
fluctuations. In the United States, one CAES 110-MWe energy storage system has been built with a cost of $\$ 410 / \mathrm{kW}$ (Succar and Williams). A new isothermal CAES design by Sustainex claims to have a production cost of approximately $\$ 25 / \mathrm{MWh}$, which is used in this model (Sustainex). A pumped hydro production cost of $\$ 20 / \mathrm{MWh}$ is used (IRENA 2012). Production costs are the total operational costs for a system, including fuel and O\&M where applicable. Table 9 displays a summary of the inputs.

Table 9. Summary of inputs.

\begin{tabular}{|c|c|}
\hline Time Frame & $7 / 1 / 2013-6 / 30 / 2014$ \\
\hline Electricity Wholesale Price & NY ISO Hourly Locational Based Marginal Pricing ${ }^{*}$ \\
\hline Nuclear Capacity & 1639.5 MWe (NY ISO 2013) \\
\hline Wind Capacity & 418.9 MWe (NY ISO 2013) \\
\hline Natural Gas Capacity & $775 \mathrm{MWe}$ \\
\hline Storage Capacity & $600 \mathrm{MWe}$ \\
\hline Wind Efficiency & $30 \%$ (IRENA 2012b) \\
\hline CAES Efficiency & 70\% (IRENA 2012c) \\
\hline Pumped Hydro Efficiency & 90\% (IRENA 2012) \\
\hline Nuclear Production Cost & $\$ 27.10 / \mathrm{MWh}$ \\
\hline Natural Gas Production Cost & \$34/MWh (NEI 2012) \\
\hline Wind Production Cost & \$10/MWh (IRENA 2012b) \\
\hline Nuclear Fuel Cost & \$7.5/MWh (NEI 2013) \\
\hline Natural Gas Fuel Cost & \$29.3/MWh (NEI 2013) \\
\hline Wind Fuel Cost & $\$ 0 / \mathrm{MWh}$ \\
\hline CAES Operational Costs & \$0.57/MWh (IRENA 2012c) \\
\hline Pumped Hydro Production Cost & \$11/MWh (IRENA 2012) \\
\hline Hydrogen Production Cost & $\$ 1.74 / \mathrm{kg}^{\times}$(Dillich, Ramsden, and Melaina 2012) \\
\hline Hydrogen Conversion & $32.9 \mathrm{kWh} / \mathrm{kg}^{++}$(Hydrogen Energy Systems LLC) \\
\hline Hydrogen Efficiency & $70 \%$ (DOE 2004) \\
\hline Hydrogen Sale Price & $\$ 8 / \mathrm{kg}(\$ 4-\$ 12 / \mathrm{kg})($ Blencoe 2009$)$ \\
\hline
\end{tabular}

"Locational Based Marginal Pricing information may be found in Appendix I.

${ }^{++}$For atmospheric electrolysis. Conversion factor for compressed electrolysis is $60 \mathrm{kWh} / \mathrm{kg}$.

${ }^{\times}$These costs do not include hydrogen storage or distribution.

\subsection{NHES Results and Discussion}

To analyze the effectiveness of SMRs in a NHES, the overall production costs, revenue, and profits associated with a SMR-wind-natural gas hybrid energy system with and without storage of nuclear energy and with hydrogen sales from excess heat. This is an attempt to demonstrate the potential savings associated with combined heat and power systems. Table 10 displays the production costs (fuel + O\&M) for the three cases resulting from the assumptions shown in Table 9. No amortized capital is included in these results. Wind, with its zero variable operating costs is used first; next, nuclear energy would be deployed, followed by natural gas for this particular system. For energy technologies like nuclear and natural gas, the variable operating costs which are used to determine the merit order are essentially the fuel costs shown in Table 9. Natural gas fuel makes up a large percentage of the production costs, and as a result, is used last in this system. More information on the merit order of energy generating technologies may be found in Appendix I. In practice, all the wind and most of the nuclear energy being produced in this model are used for electricity, while the natural gas is only deployed to meet additional intermediate or peak demand. The Excel inputs and general methodology may be seen in Appendix J.

Table 10. Comparison of Results

\begin{tabular}{|l|l|}
\hline Variable and Fixed Operational Costs & \\
\hline Base Case: Electric-Only & $\$ 27 / \mathrm{MWh}$ \\
\hline
\end{tabular}




\begin{tabular}{|l|l|}
\hline Storage Case: CAES & $\$ 25 / \mathrm{MWh}$ \\
\hline Storage Case: Pumped Hydro & $\$ 25 / \mathrm{MWh}$ \\
\hline Hydrogen Production Case & $\$ 28 / \mathrm{MWh}$ \\
\hline
\end{tabular}

From the results in Table 10, the storage cases demonstrate lower operating costs despite having added additional storage devices with their own inherent operating costs; this is due to the nuclear energy being stored and used to help meet peak demand, rather than using the more costly natural gas to meet the demand. In the Hydrogen Production Case, the cost increases with the addition of hydrogen production facilities. It should be noted that these are merely costs, and the profits must also be analyzed to see the effects of different systems.

The cost and profit shares of the revenue for the base case and storage case are shown in Figure 26, demonstrating that approximately $50 \%$ of the revenue is profit. In the storage case, the additional revenue generated from lower cost wind and nuclear energy to cover the peak demand from storage improves the overall costs. The cost share of the revenue is reduced by approximately $3.6 \%$, despite the fact that storage costs were added. Alternatively, the profit share of the revenue increased by nearly $4 \%$.

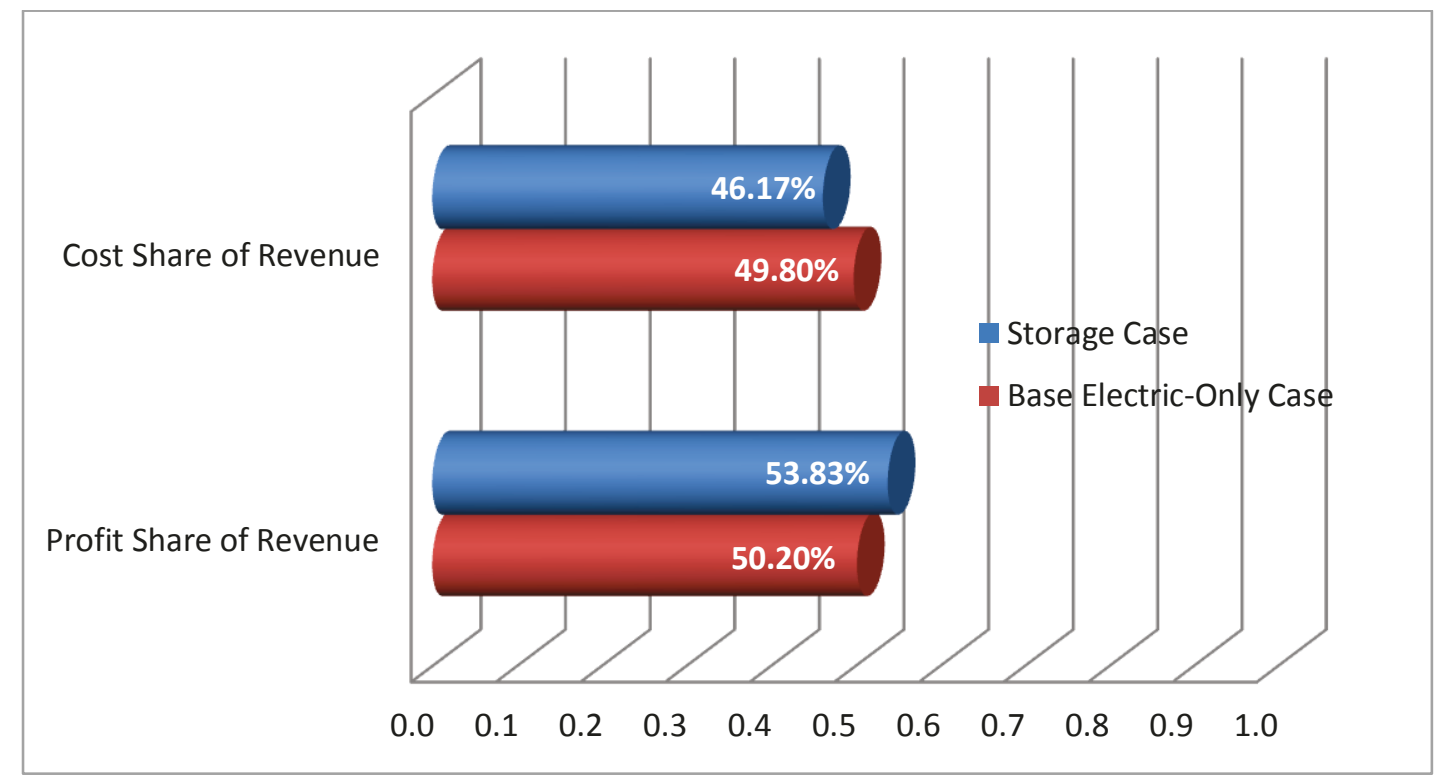

Figure 26. Profit and cost shares for Base Case and Storage Case with CAES.

The revenue and profit shares for the hydrogen production cases are displayed in Figure 27. In this case, the system was designed to generate approximately $10-11 \%$ of the revenue from hydrogen sales. $78 \%$ of the hydrogen revenue is profit, while only $50 \%$ of the electric revenue is profit. This demonstrates why recovering the excess heat energy to produce hydrogen is a valuable enterprise. 


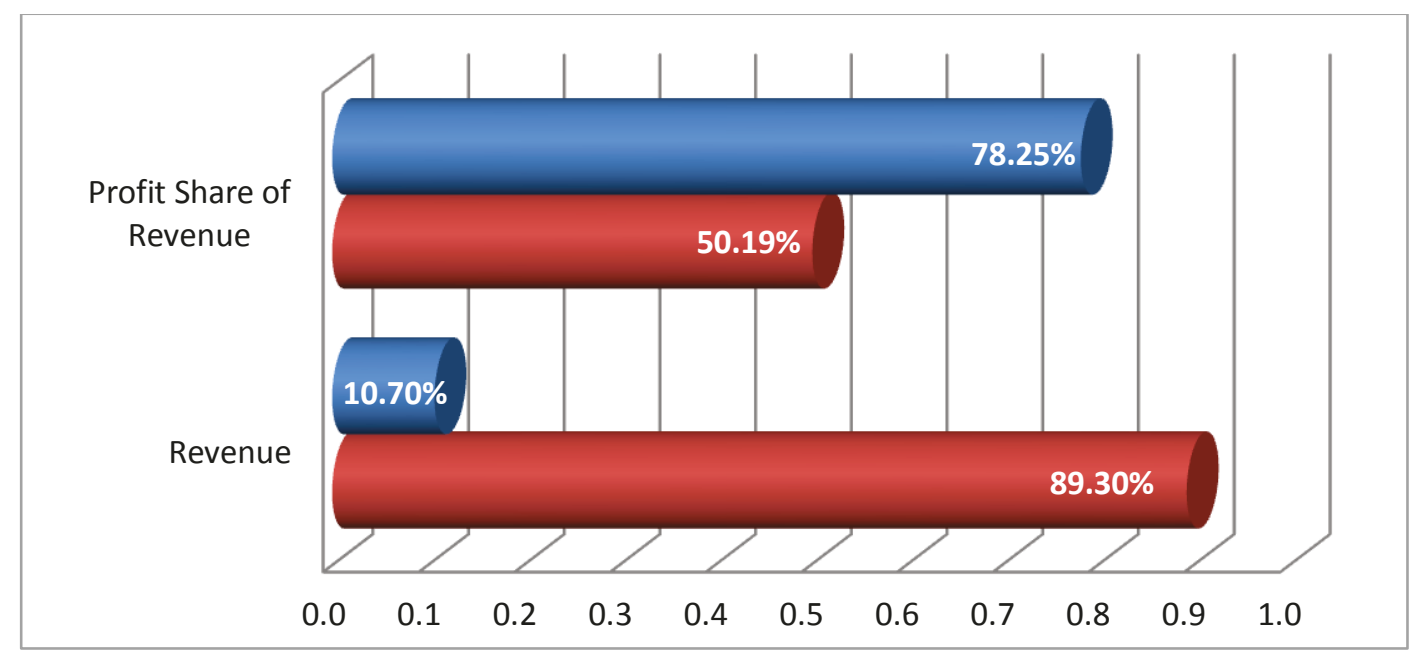

Figure 27. Profit and revenue shares for Hydrogen Production Case.

\section{SMR COMPETITION AND CLIMATE}

\subsection{Competition}

Because the price of natural gas is at a historically low point, the last few years have seen a large increase in the development of natural gas plants. In 2011, natural gas prices ranged from $\$ 60$ to $\$ 80 / \mathrm{MWh}$ (Rosner and Goldberg 2011). Presently, the prices in 2014 range from $\$ 65 / \mathrm{MWh}$ to $\$ 130 / \mathrm{MWh}$ (NEI 2014). Competition is the primary driver of the electricity generation market, and as a result, will have to warrant nuclear energy development. Because $90 \%$ of the natural gas production costs are fuel costs, fluctuations in the future will likely make renewable technologies and nuclear energy more competitive (NEI 2014). Furthermore, now that natural gas accounts for more than $30 \%$ of electricity generation in the United States, grid reliability may be an additional factor that supports nuclear development (NEI 2014). Historically, natural gas fuel costs have been much greater than nuclear fuel costs and have fluctuated significantly. Figure 28 demonstrates the long term stability of nuclear fuel costs. Furthermore, Figure 29 shows why the fluctuations greatly affect the natural gas production costs, as fuel accounts for almost $90 \%$ of these costs. On the other hand, fluctuations in nuclear fuel prices would not impact the production costs as substantially. 


\section{Monthly Fuel Cost to U.S. Electric Utilities 1995 - 2012, In 2012 cents per kilowatt-hour}

250

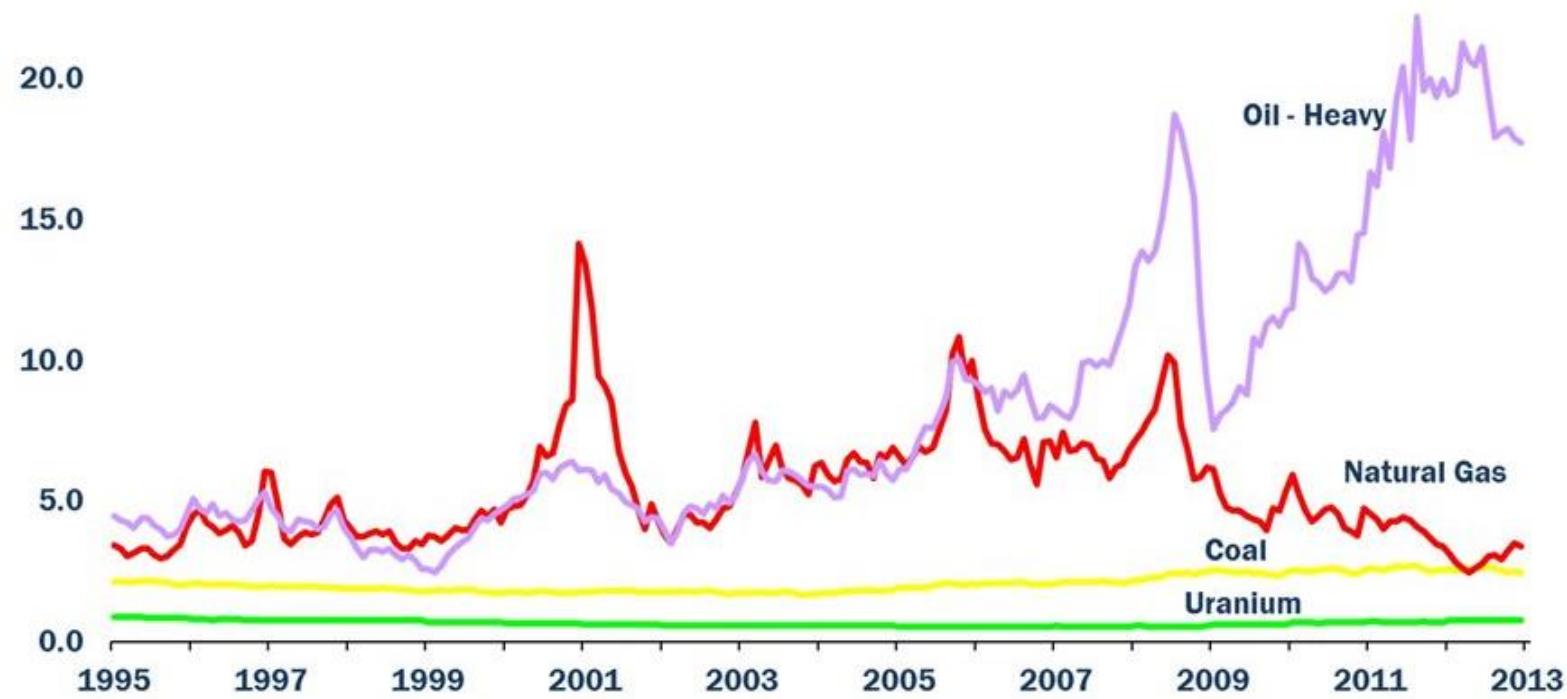

Figure 28. Electric Utility Fuel Costs for Oil, Natural Gas, Coal, and Nuclear Energy (NEI 2013b).

\section{Fuel as a Percentage of Electric Power Production Costs}

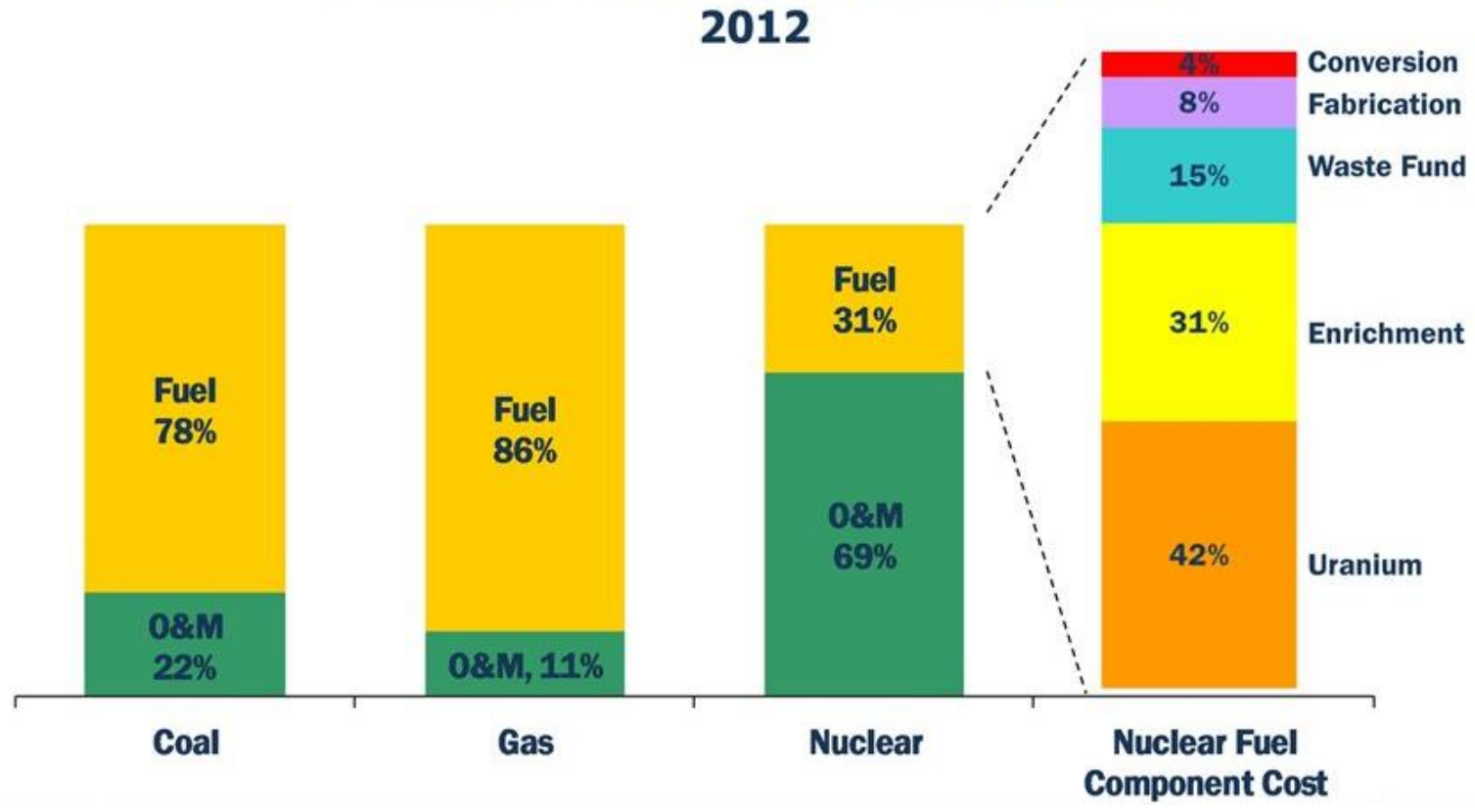

Figure 29. Breakdown of Fuel and O\&M percentages of the production costs for coal, natural gas, and nuclear energy (NEI 2013c). 


\subsection{Investment Risks}

For a project to be pursued, all the risks must be weighed. For electricity generation, there are significant regulatory, political, technological, financial, resource, safety, supply, proliferation, and decommissioning risks (IAEA 2008). Regulatory risks involve changes to environmental or safety regulations that may result in significantly increased costs to produce power as well as electricity market conditions dictated by regulatory bodies. Political risks involve the social standing of nuclear power at the local, state, and federal levels. Technological risks occur when new technologies or systems are developed. Incorporating new reactor designs would result in increased technological risk. However, standardizing a large number of reactors would help mitigate this risk (IAEA 2008).

Changes to the financial market, such as changes in tax rates and fiscal policy may greatly impact higher capital technologies (IAEA 2008). High capital cost projects are much more sensitive to fluctuations in interest rates and cost overruns (IAEA 2008). They are less able to respond to changing market conditions, as well. The availability of resources, including both skilled personnel and fuel supply, are risk factors (IAEA 2008). The fuel supply risk is particularly low for reactors, especially if the refueling cycle is longer. Fossil fuels, on the other hand, have a much greater risk, as suggested in the availability of diesel fuel in the Alaska case study. Safety and health is the major risk for nuclear energy, as the ultimate goal is to provide clean air electricity while preventing harm to the public via contamination or radiation release during accidents (IAEA 2008). Decommissioning risks also play into safety, as restoration of the site may be difficult if any accidents have occurred (IAEA 2008). Additionally, permanent waste storage may be difficult to accommodate, particularly if the political or regulatory environments have changed. Finally, proliferation is a concern during operation and storage.

All of these risks must be taken into account when assessing the feasibility of any proposed project, but many apply specifically to nuclear power. The financial risks are particularly important when estimating construction time and renewable energy on the market, which would heavily influence overall project costs and the possible revenue in a given region.

\section{POLITICAL ENVIRONMENT}

Government involvement is crucial in multiple stages of technological development. Initial involvement should support research and development and even demonstration of a new concept. In energy technologies, this is done through Department of Energy support and research. This alone is not enough to make a new technology attractive to utility companies. Policy must dictate conditions that would make these technologies a good business investment, particularly if high upfront capital costs are necessary (IAEA 2008). This may be done through many methods such as asset ownership, equity participation, risk sharing, electricity tariffs to support construction, etc. (IAEA 2008). Other incentives, like loan guarantees or guaranteed power purchases, may prove helpful (IAEA 2008). Capital cost and production cost incentives may also help mitigate the risks associated with high capital costs (Rosner and Goldberg 2011). Some incentives are already in play or have been used in the past. For example, production incentives are provided for up $6 \mathrm{GWe}$ of installed power prior to 2019, according to the EIA (EIA 2014b).

Tax credits are another important method to support the production of renewable energies and nuclear energy (Rosner and Goldberg 2011). Nuclear energy has a huge advantage over fossil fuels, in its reduction in carbon emissions. However, "the advantage of nuclear energy in alleviating the risk of climate change will not favor market penetration of nuclear products through advanced non-electric applications of nuclear power as long as energy policies internalizing the value of carbon and other pollutants are not implemented" (IAEA 2007). Other countries which apply value to the effects on global warming, material damage, crop loss, and even health effects support the development of renewable and nuclear energies. Germany, for example, does this, and the external costs for producing each power method are illustrated in Figure 30 (IAEA 2007). The overall costs for producing power are shown in 
Figure 31 (IAEA 2007). These external costs are site specific and will help dictate which energy method is best for that particular area.

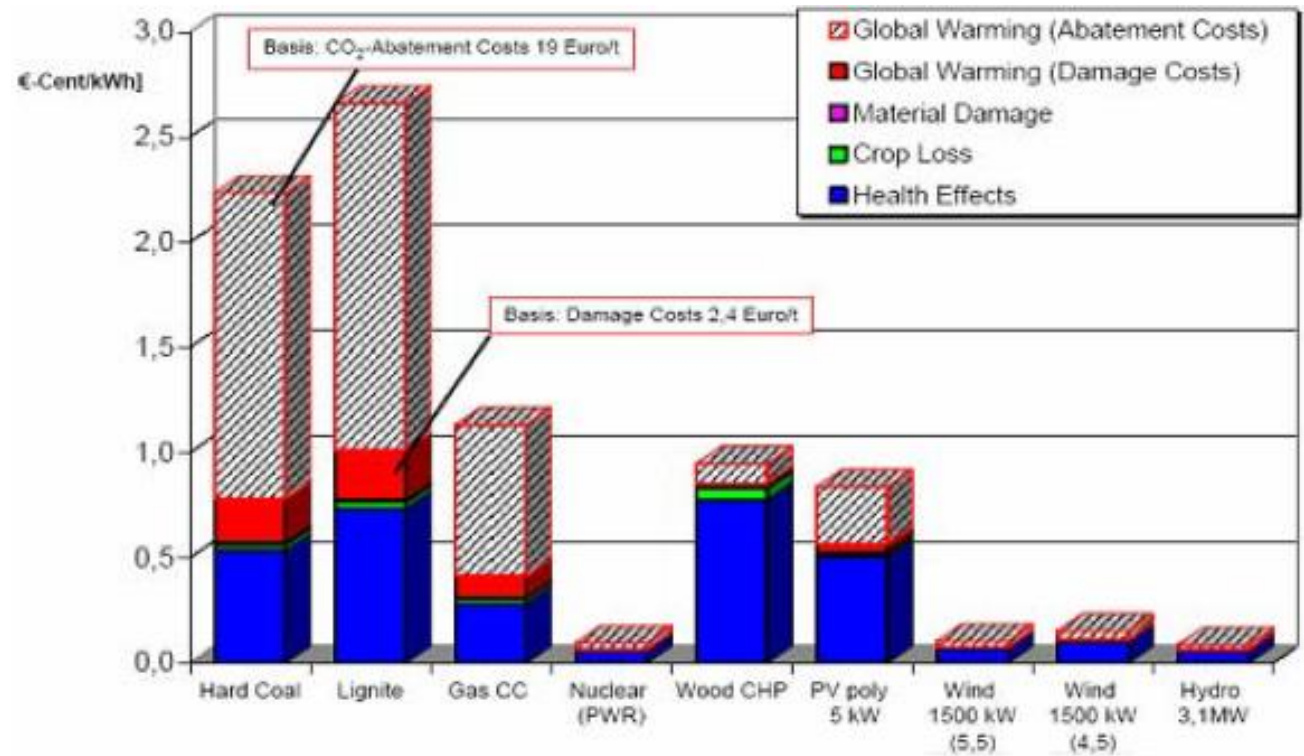

Figure 30. External cost comparison in Germany, including global warming costs (IAEA 2007).

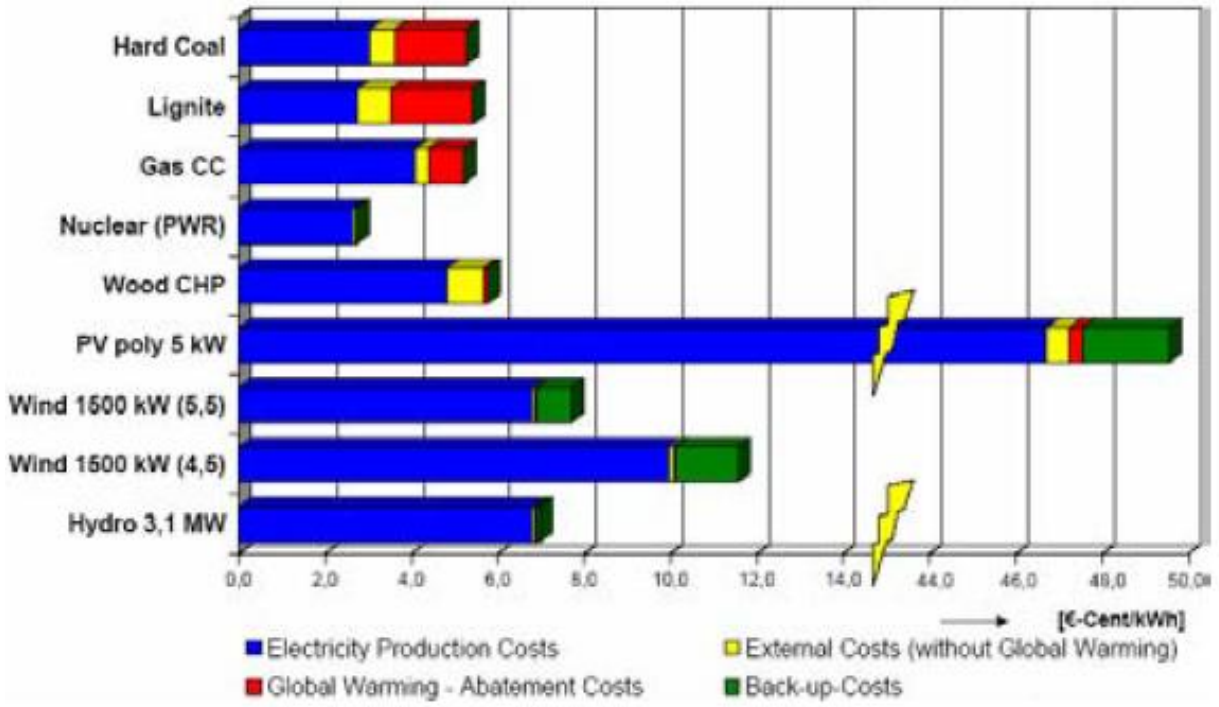

Figure 31. Total electricity generating costs for different technologies in Germany, including external costs (IAEA 2007).

Some policies which apply to the United States may be used to further development policies to address external factors. However, the "Kyoto Protocol in February 2005 does create incentives that can benefit nuclear power, depending on how they are translated into national policies. The Protocol's emission trading provisions effectively give a cash value to unused emission allowances in the Russian Federation, for example, and the European Trading Scheme (ETS) creates incentives favorable to nuclear power in at least those European countries where implementation policies are not specifically biased against nuclear power" (IAEA 2007). Government involvement does not just entail domestic financial policies. It also entails the development of global relationships to further advanced research with multinational support. 


\subsection{Licensing}

Other nations have attempted to amend the philosophy and methodology of their licensing process in order to facilitate licensing and construction of newer reactor designs such as HTRs. The South African National Nuclear Regulator has made changes to accommodate the ESKOM Pebble Bed Modular Reactor (PBMR) (Bester and Hill 2008). In this particular framework, the NNR utilizes a multi-stage process, in which they are directly involved in certain steps in the design process. Because no international design basis and safety criteria have been developed for PBMRs, it is necessary that the designers provide sufficient information for the NNR to develop them. The NNR has attempted to create technology neutral defense-in-depth requirements and to detail the specific future work/research into the design that would be necessary to meet their standards. This framework ultimately utilizes an iterative process between design and safety for FOAK designs (Bester and Hill 2008).

With the increased attention to SMRs, a study into the potential SMR Finnish regulatory framework was developed in order to demonstrate the applicability of the framework to SMRs and to provide recommendations to facilitate the future licensing of SMRs. The main recommendation entails the addition of a Standard Design Certification of Module (SDCM), which would minimize the regulatory oversight requirements while construction is underway (from the Construction License to Operating License Phases) (Soderholm 2013). SMRs high level designs for the module and its safety systems can be standardized much more readily than a large reactor. The long term goal presented is an international licensing process incorporating the SDCM (Soderholm 2013).

The addition of an SDCM may be feasible in Finland; however, it may not be a viable option in other regulatory frameworks. "The NRC licensing process, which has been used as a basis for regulatory frameworks in many countries, emphasizes the predictable process. This prescriptive licensing process includes very detailed requirements, which can be seen as both beneficial and challenging. The benefit comes from the predictability of the process and requirements, while the challenge is the heavy and time consuming process in which the requirements are formulated, design by design" (Soderholm 2013). The UK licensing process may be seen at the other end of the licensing spectrum; this framework "focuses on a goal setting approach, which brings flexibility to the licensing process. This approach sets only the high level regulations, while the licensee is obligated to present the safety case and therefore the required safety level fulfillment" (Soderholm 2013). More flexible frameworks may provide a better opportunity for SDCM incorporation.

In the NRC framework, SMR designs utilizing prior LWR technologies and newer passive and safety systems, such as those licensed in the AP1000 design, do still have a high probability of obtaining the necessary licenses. It is the more innovative or advanced designs, which much demonstrate their technologies, materials, etc. Perkowski summarizes the R\&D requirements, which must be undertaken as part of the design process in order for advanced designs to eventually obtain licensing within the United States:

- "Develop evaluation assessment methods for advanced SMR technologies;

- Develop and test materials, fuels, and fabrication techniques;

- Resolve key regulatory issues identified by NRC and industry; and

- Develop advanced instrumentation, controls, and human-machine interfaces" (Perkowski 2012).

NRC Commissioner Magwood described the key focus areas for an NRC licensing review as (Magwood 2001):

- Reactor safety

- Radiation safety for employees and the public

- Plant security and protection from sabotage and security threats 
The Report to Congress on Small Modular Nuclear Reactors details how SMRs are specifically designed to meet the aforementioned general review categories. SMRs have been designed to eliminate initiating events entirely, where possible, and to mitigate the consequences of any events that may not be eliminated. This is often achieved through reduced components, added simplicity, and enhanced passive safety features. The following is taken from the Report to Congress on Small Modular Nuclear Reactors and details four major components of the report regarding initiating events, mitigating systems, barrier integrity, and emergency preparedness:

"Initiating Events-Most SMR designs and concepts are simpler than existing light-water reactor (LWR) designs. This reduces the number of systems required to provide and support the heat transport and electrical generation of the plant. In addition, inherent safety features reduce the number and complexity of accident mitigation systems. The resulting reduction in mechanical components and associated control systems greatly reduces the potential for equipment failure that leads to plant shutdowns, large changes in the plant's power output, or accidents.

Mitigating Systems-SMR designs typically take a different approach to mitigating accidents by using the design to reduce the potential for an accident occurring and to reduce the severity if one does occur. For example, a negative temperature coefficient is maintained for the reactor core, and passive and inherent safety systems are used to remove the human error element that can potentially affect proper plant response to accident conditions.

Burrier Integrity-Some SMR designs rely on the integrity of the fuel to retain fission products under all postulated conditions, instead of relying on a pressure-retaining containment building to contain any fission products released as the result of a reactor accident. This makes verification of fuel integrity extremely important because, unlike a containment building that can be periodically leak-rate tested, verification of fuel integrity after the initial fabrication is difficult. However, if fuel performance can be guaranteed, the SMR can be much simpler and easier to maintain through the elimination of a conventional containment building.

Emergency Preparedness-An SMR will still have comprehensive emergency plans to respond to a possible accident. However, the extent of the emergency plan will be based on the worst-case, source-term for radioactive release estimated by the accident analysis. It is possible that evacuation of the public beyond the site boundaries will not be necessary because of the estimated small-source term.

- Occupational Radiation Safety and Public Radiation Safety: These regulations will not change.

- Physical Protection: Nuclear Plants are required to guard vital plant equipment. There will be fewer attractive materials easily accessible with most SMR designs" (Magwood 2001).

\section{CONCLUSION}

The future of small modular reactors in the United States will ultimately be determined by market competition, the ability to obtain construction and operating licenses from the Nuclear Regulatory Commission in a reasonable time frame, and the political environment. This report presents scenarios in which SMRs may be made more attractive and competitive with load following generation technologies, such as natural gas; conveys the overall effects on cost that result from a mixed energy portfolio with and without storage; and demonstrates how both storage and industrial process heat applications may add to the overall profit margins in nuclear hybrid energy systems.

Obtaining licenses in a reasonable time frame would enable designs to progress from the preliminary stages through construction without significant investment losses. This is unlikely for newer unproven designs or more specifically for non-LWR designs. As a result, the clear path forward for SMRs is the licensing, construction, and operation of Generation III+ light-water SMRs designed with enhanced passive safety features and defense-in-depth features. These units would demonstrate the maturity of 
SMR inherent safety and passive design features and may result in a reduction in emergency planning requirements, necessary human intervention during normal operations and initiating events, and staffing requirements; they would also show improved risk mitigation and proliferation resistance.

This first generation of SMR units in the United States would also support the addition of new renewable energy technologies, reducing greenhouse gas emissions. Furthermore, SMRs may be competitive with both large conventional reactors and with natural gas plants. As shown in this report, SMRs actually have a higher return on investment than large conventional rectors and are far more flexible to deploy. Natural gas prices have seen drastic fluctuations in the past and will continue to in the future, making future energy planning extremely difficult; SMRs, on the other hand, do not see these fuel price fluctuations and are therefore a much more stable, long term, clean air energy technology.

\section{REFERENCES}

G. Locatelli, C. Bingham and M. Mancini, "Small modular reactors: A comprehensive overview of their economics and strategic aspects.," Progress in Nuclear Energy, vol. 75, pp. 75-85, 2014.

“Current Status, Technical Feasibility and Economics of Small Nuclear Reactors," NEA/OECD, 2011.

"Value of Energy Diversity Fact Sheet," Nuclear Energy Institute, 2014.

IAEA 2007 “Advanced Applications of Water Cooled Nuclear Power Plants,” IAEA, 2007.

R. Rosner and S. Goldberg, "Small Modular Reactors - Key to Future Nuclear Power Generation in the U.S.," University of Chicago: Energy Policy Institute at Chicago, 2011.

“NuScale's Regulatory Process Status,” NuScale Power, 2014. [Online]. Available: http://www.nuscalepower.com/nrcinteraction.aspx.

"B\&W mPower," Nuclear Regulatory Commission, 2014. [Online]. Available: http://www.nrc.gov/reactors/advanced/mpower.html.

C. L. Kling and M. D. Carelli, "IRIS Licensing Status,” IEA/INIS, 2014. [Online]. Available: http://inis.iaea.org/search/search.aspx?orig_q=RN:44064691.9

"Cost Estimating Guidelines for Generation IV Nuclear Energy Systems," Generation IV International Forum Economic Modeling Working Group, 2006.

G. Rothwell, "The Cost Economics of Advanced Nuclear Power Technologies with Application to Modular Sodium Fast Recycling Reactors," Stanford, 2007.

V. Kuznetsov, "Options for small and medium sized reactors (SMRs) to overcome loss of economies of scale and incorporate increased proliferation resistance and energy security," Progress in Nuclear Energy, vol. 50, pp. 242-250, 2008.

M. D. Carelli, B. Petrovic and C. Mycoff, "Economic Comparison of Different Size Nuclear Reactors," Westinghouse, 2007.

E. Wilton, "A Conceptual Framework for the Comparison of Small Modular Reactors based on Passive Safety Features, Proliferation Resistance, and Economic Potential (Master's Thesis)," Utrecht University, 2012.

M. D. Carelli, B. Petrovic, N. Cavlina and D. Grgic, "IRIS (International Reactor Innovative and Secure) - Design Overview and Deployment Prospects," in Nuclear Energy for New Europe, 2005.

"Nuclear Energy Series: Design Features to Achieve Defence in Depth in Small and Medium Sized Reactors," IAEA, 2009.

M. D. Carelli, L. E. Conway, L. Oriani, B. Petrovic, C. V. Lombardi, M. E. Ricotti, A. Barroso, J. M. Collado, L. Cinotti, N. E. Todreas, D. Grgic, M. M. Moraes, R. D. Boroughs, H. Ninokata, D. T. 
Ingersoll and F. Oriolo, "The design and safety features of the IRIS reactor," Nuclear Engineering and Design, 2004.

"Status and Near-Term Prospects of Small and Medium Sized Reactors," IAEA, 2010.

"Status of innovative small and medium sized reactor designs 2005," IAEA, 2006.

"High Temperature Gas Reactors: Assessment of Applicable Codes and Standards," Pacific Northwest Naitonal Laboratory, 2011.

“The High Temperature Gas-Cooled Reactor Next Generation Nuclear Energy," Next Generation Nuclear Plant, Idaho National Laboratory.

"Liquid Metal Cooled Reactors: Experience in Design and Operation,” IAEA, 2007.

M. Schneider, A. Froggatt and J. Hazemann, "World Nuclear Industry Status Report,” 2012.

Grubler, "The costs of the French nuclear scale-up: A case of negative learning by doing," Energy Policy, vol. 38, pp. 5174-5188, 2010.

"Reduction of Capital Costs of Nuclear Power Plants," IEA/NEA/OECD, 2000.

M. Carelli, P. Garrone, G. Locatelli, M. Mancini, M. Mycoff and P. Trucco, "Economic features of integral, modular, small-to-medium size reactors," Progress in Nuclear Energy, vol. 52, pp. 403-414, 2010.

“Technology Learning Curve FOAK to NOAK," National Energy Technology Laboratory, 2013.

L. Reid, "Modelling Modularity Impacts on Nuclear Power Plant Costs," Oak Ridge National Laboratory, 2003.

"Structuring Nuclear Projects for Success An Analytic Framework," World Nuclear Association.

"Projected Costs of Generating Electricity," IEA/NEA/OECD, 2010.

Carelli, Competitiveness of Small-Medium, new generation Reactors: a comparative study on Capital and O\&M Costs (Presentation), 2008.

W. Short, D. J. Packey and T. Holt, "A Manual for the Economic Evaluation of Energy Efficiency and Renewable Energy Technologies," National Renewable Energy Laboratory, 1995.

“Advanced Nuclear Power Reactors,” World Nuclear Association, 2014. [Online]. Available: http://www.world-nuclear.org/info/Nuclear-Fuel-Cycle/Power-Reactors/Advanced-Nuclear-Power -Reactors/.

“Advanced Boiling Water Reactor,” Hitachi-GE Energy, Ltd.

“2014 Tax rates, schedules, and contribution limits," Putnum Investments, 2014.

“Average Retail Price of Electricity to Ultimate Customers," U.S. Energy Information Administration. [Online]. [Accessed 2014].

"Hawai'i is the Most Fossil Fuel Dependent State in the Nation," Hawai'i Powered Clean Energy Initiative, 2010. [Online]. Available: http://www.hawaiicleanenergyinitiative.org/.

"Guide to the Successful Implementation of State Combined Heat and Power Policies," Industrial Energy Efficiency and Combined Heat and Power Working Group, State and Local ENergy Efficiency Action Network, 2013.

McKinsey, "Unlocking Energy Efficiency in the U.S. Economy," McKinsey \& Company, 2009.

"Basic Information," U.S. EPA Combined Heat and Power Partnership, 2013. [Online]. Available: http://www.epa.gov/chp/basic/. 
"Understanding Base Load Power What it is and Why it Matters," NY Affordable Reliable Electricity Alliance, 2008.

C. Forsberg and E. Schneider, "Increasing base-load LWR revenue with heat storage and variable electricity output".

S. Daniels, "Why Exelon may cut its Illinois nuke output," Chicago Business, 2013. [Online]. Available: http://www.chicagobusiness.com/article/20130620/NEWS11/130619723/why-exelon-may-cut-itsillinois-nuke-output.

M. Ruth, O. Zinaman, M. Antkowiak, R. Boardman, R. Cherry and M. Bazilian, "Nuclear-renewable hybrid energy systems: Opportunities, interconnections, and needs," Energy Conversion and Management, vol. 78, pp. 684-694, 2014.

A. Akhhil, G. Huff, A. Currier, B. Kaun, D. Rastler, S. Chen, A. Cotter, D. Bradshaw and W. Gauntlett, "DOE/EPRI 2013 Electricity Storage Handbook in Collaboration with NRECA," Sandia National Laboratory, 2013.

R. Tipps, E. Schneider, C. van der Hoeven, and C. Forsberg (1), "Impact of Energy Storage on Nuclear Electricity Economics."

R. Tipps, E. Schneider, C. van der Hoeven, and C. Forsberg (2), "Simplified Market Analysis of Nuclear Energy Storage."

T. Anjum, "Peak Electricity Demand and the Feasibility of Solar PV in the Greater Boston Area (Bachelor's Thesis)," Worcester Polytechnic Institute, 2013.

"New York Controlled Area Load Zones," NY ISO, [Online]. Available: http://www.nyiso.com/public/webdocs/markets_operations/market_data/zone_maps_graphs/nyca_ zonemaps.pdf.

“2013 Load and Capacity Data Gold Book,” NY ISO. 2013.

“Zone Maps,” NY ISO, [Online]. Available: http://www.nyiso.com/public/markets_operations/market_data/maps/index.jsp.

“Custom Reports,” NY ISO, 2014. [Online]. Available: http://www.nyiso.com/public/markets_operations/market_data/custom_report/index.jsp?report=int _rt_actual_load.

S. Succar and R. Williams, "Wind Coupled to To Compressed Air Energy Storage (CAES) For Baseload Power Generation," Princeton Environmental Institute.

Isothermal CAES for Grid-Scale Applications (Presentation), Sustainex.

“Renewable Energy Technologies: Cost Analysis Series Hydropower,” IRENA, 2012.

IAEA, Nuclear Energy Series: Financing of New Nuclear Power Plants. 2008.

P. Bester and T. Hill, South African Licensing Framework for the Pebble Bed Modular Reactor, Proceedings of the $4^{\text {th }}$ International Topical Meeting on High Temperature Reactor Technology (2008) HTR2008-58185.

B. Dixon, K. Williams, J. Blink, J. Carter, E. Danko, F. Ganda, T.J. Harrison, E. Schneider, S. Sheetz, T. Wood, Advanced Fuel cycle Cost Basis (2012 Addendum), INL Fuel Cycle Research and Development, 2013.

J. Steward, A. Lamont, G. Rothwell, C. Smith, E. Greenspan, N. Brown, A. Barak, An Economic Analysis of Generation IV Small Modular Reactors, LLNL, 2002.

W. Magwood IV, Report to Congress on Small Modular Nuclear Reactors, DOE Office of Nuclear Energy, Science, and Technology, 2001.

G. Blencoe, Cost of hydrogen from different sources, Hydrogen Car Revolution, 2009. 
"Renewable Energy Technologies: Cost Analysis Series Wind Power. 2012b.

Nuclear Hydrogen R\&D Plan, DOE, 2004.

Hydrogen Energy Systems LLC website: http://heshydrogen.com/hydrogen-fuel-cost-vs-gasoline/.

Electricity Storage and Renewables for Island Power, IRENA, 2012c.

U.S. Electricity Production Costs, NEI, 2012. Online:

http://www.nei.org/Knowledge-Center/Nuclear-Statistics/Costs-Fuel,-Operation,-Waste-Disposal-Life-Cy cle/US-Electricity-Production-Costs.

S. Dillich, T. Ramsden, and M. Melaina, Hydrogen Production Cost Using Low-Cost Natural Gas, DOE Hydrogen and Fuel Cells Program Record, 2012.

"Locational Based Marginal Pricing..." (Presentation), NY ISO website: http://www.nyiso.com/public/services/market_training/online_resources/lbmp_online.pdf.

Soderholm, Kristiina. Licensing Model Development for Small Modular Reactors (SMRs) - Focusing on the Finnish Regulatory Framework. Lappeenranta University of Technology. 2013.

Perkowski, Joseph. Small Modular Reactors: Institutional Assessment. INL 2012.

Levelized Cost and Levelized Avoided Cost of New Generation Resources in the Annual Energy Outlook 2014, U.S. Energy Information Administration, 2014b.

C. Forsberg and E. Schneider, "Increasing Baseload LWR Revenue with Heat Storage and Variable Electricity Output," in the June 2014 ANS Transactions for the Reno meeting.

U.S. Electricity Production Costs and Components, Nuclear Energy Institute, 2013. [Online]. Available: http://www.nei.org/Knowledge-Center/Nuclear-Statistics/Costs-Fuel,-Operation,-WasteDisposal-Life-Cycle/US-Electricity-Production-Costs-and-Components

Monthly Fuel Cost to US Electric Utilities, Nuclear Energy Institute, 2013b. [Online]. Available: http://www.nei.org/Knowledge-Center/Nuclear-Statistics/Costs-Fuel,-Operation,-Waste-Disposal-LifeCycle/Monthly-Fuel-Cost-to-US-Electric-Utilities

Fuel as a Percent of Production Costs, Nuclear Energy Institute, 2013c. [Online]. Available: http://www.nei.org/Knowledge-Center/Nuclear-Statistics/Costs-Fuel,-Operation,-WasteDisposal-Life-Cycle/Fuel-as-a-Percent-of-Production-Costs 


\section{Appendix A NPP Detailed Cost Breakdown (GIF Code of Accounts)}

Table A1: NPP Code of Accounts [12]

\begin{tabular}{|c|c|}
\hline Account Number & Account Title \\
\hline 1 & Capitalized Pre-Construction Costs (CPC) \\
\hline 11 & Land and Land Rights \\
\hline 12 & Site Permits \\
\hline 13 & Plant Licensing \\
\hline 14 & Plant Permits \\
\hline 15 & Plant Studies \\
\hline 16 & Plant Reports \\
\hline 17 & Other Pre-Construction Costs \\
\hline 18 & Other Pre-Construction Costs \\
\hline 19 & Contingency Pre-Construction Costs \\
\hline 2 & Capitalized Direct Costs (CDC) \\
\hline 21 & Structures and Improvements \\
\hline 22 & Reactor Equipment \\
\hline 23 & Turbine-Generator Equipment \\
\hline 24 & Electrical Equipment \\
\hline 25 & Heat Rejection System \\
\hline 26 & Miscellaneous Equipment \\
\hline 27 & Special Materials \\
\hline 28 & Simulator \\
\hline 29 & Contingency Direct Costs \\
\hline \multicolumn{2}{|l|}{ DIRECT COST } \\
\hline 3 & Capitalized Indirect Services Cost (CIC) \\
\hline 31 & Field Indirect Costs \\
\hline 32 & Construction Supervision \\
\hline 33 & Commissioning and Start-up Costs \\
\hline 34 & Demonstration Test Run \\
\hline TOTAL FIELD COST & \\
\hline 35 & Design Services Offsite \\
\hline 36 & PM/CM Services Offsite \\
\hline 37 & Design Services Onsite \\
\hline 38 & PM/CM Services Onsite \\
\hline 39 & Contingency Support Services \\
\hline \multicolumn{2}{|l|}{ BASE COST } \\
\hline 4 & Capitalized Owner Cost (COC) \\
\hline 41 & Staff Recruitment and Training \\
\hline 42 & Staff Housing \\
\hline 43 & Staff Salary Related Costs \\
\hline 44 & Other Owner Capitalized Costs \\
\hline 49 & Contingency Operations Costs \\
\hline 5 & Capitalized Supplementary Costs (CSC) \\
\hline 51 & Shipping and Transportation Costs \\
\hline 52 & Spare Parts \\
\hline
\end{tabular}




\begin{tabular}{|cc|}
\hline \multicolumn{2}{|c|}{ Account Number } \\
53 & Taxes \\
54 & Insurance \\
55 & Initial Fuel Core Load \\
58 & Decommissioning Costs \\
59 & Contingency Supplementary Costs \\
OVERNIGHT COST & \\
\hline 6 & Capitalized Operations \\
61 & Escalation \\
62 & Fees \\
63 & Interest During Construction \\
64 & Contingency Financial Costs \\
TOTAL CAPITAL INVESTMENT COST (TCIC) \\
\hline
\end{tabular}




\section{Appendix B \\ Real and Nominal Discount Rate Methods}

The discount rate is an extremely important component in calculating an appropriate Net Present Value and Levelized Cost of Electricity that will define whether an investment should be made. It represents the interest, or that at which the investment is made. If inflation is accounted for in this rate, then this is a nominal interest. The real discount rate reflects the interest rate for investments without inflation.

Discount rates are applied to annual cash flows, so that the present value of financial losses may be determined. This is due to the fact that money, even one year from now, is not valued the same as money in the present, as a result of interest rate and/or inflation losses. As mentioned in the body of this report, the real discount rate is calculated from the WACC, which includes the interest rate (or cost of debt), cost of equity, and shares of both debt and equity for a particular investment. For a 50\%-50\% share of debt and equity and costs of debt and equity of $10 \%$, the real discount rate is $7.32 \%$. This rate is applied to each annual cash flow to discount it based on the number of years in the future the cash flow takes place. The equation below displays how to discount an annual cost with the real discount rate.

$$
\text { Discounted Cost }=\frac{\text { Total Cost }}{\left(1+r_{d}\right)^{\text {Year-1 }}}
$$

For this particular example, sample incomes and costs are shown in the table below. The discounted cost for year 5 at the $7.32 \%$ real discount rate may be calculated as:

\begin{tabular}{ccccc}
\multicolumn{5}{c}{ Discounted Cost $=\frac{\$(345,545,095.24)}{(1+0.0732)^{4}}=\$(260,513,032.18)$} \\
Year & Cash Inflows & $\begin{array}{c}\text { Cash Outflows } \\
\text { (costs) }\end{array}$ & $\begin{array}{c}\text { Cumulative } \\
\text { Cash Flows }\end{array}$ & Discounted Costs \\
0 & - & $(302,813,815.24)$ & $(302,813,815.24)$ & $\$(302,813,815.24)$ \\
1 & - & $(302,813,815.24)$ & $(605,627,630.48)$ & $\$(282,167,418.75)$ \\
2 & - & $(302,813,815.24)$ & $(908,441,445.72)$ & $\$(262,928,731.10)$ \\
3 & $139,073,760.00$ & $(345,545,095.24)$ & $(1,114,912,780.96)$ & $\$(279,574,961.37)$ \\
4 & $139,073,760.00$ & $(345,545,095.24)$ & $(1,321,384,116.20)$ & $\$(260,513,032.18)$ \\
5 & $139,073,760.00$ & $(345,545,095.24)$ & $(1,527,855,451.44)$ & $\$(242,750,779.99)$ \\
6 & $278,147,520.00$ & $(388,276,375.24)$ & $(1,637,984,306.68)$ & $\$(254,172,200.01)$ \\
7 & $278,147,520.00$ & $(388,276,375.24)$ & $(1,748,113,161.92)$ & $\$(236,842,277.28)$ \\
8 & $278,147,520.00$ & $(388,276,375.24)$ & $(1,858,242,017.16)$ & $\$(220,693,940.19)$ \\
9 & $417,221,280.00$ & $(431,007,655.24)$ & $(1,872,028,392.40)$ & $\$(228,278,813.16)$ \\
10 & $417,221,280.00$ & $(431,007,655.24)$ & $(1,885,814,767.64)$ & $\$(212,714,348.63)$
\end{tabular}

Different values are discounted based on what is being calculated. For NPV, the net cash flow, or Cash Inflows - Cash Outflows, are discounted. For LCOE, the annual Cash Outflows and the electricity generation are each discounted. 


\section{Appendix C NPV Method}

Discounting is a method of calculating the present value of future monies. Real or nominal discount rates may be utilized to discount all future cash flows to the present values. The WACC may also be used for this discount rate. Once all future cash flows for a particular project lifetime have been expressed in current dollars, the summation of all terms provides the net present value [66].

$$
N P V=\sum \text { (Present Value of Cash Inflows - Present Value of Cash Outflows) }
$$

Ultimately, the NPV provides insight into the potential investment returns by estimating the worth of a project, i.e. how much money could be made off of a project. The higher the NPV, the better the investment is, and a negative NPV means money could be lost in this investment. In general, the following is applicable for analyzing the NPV [66]:

$N P V>0$, accept the investment

$N P V<0$, reject the investment

$N P V=0$, the investment is marginal 


\section{Appendix D IRR}

The Internal Rate of Return represents the estimated return on an investment. It is the discount rate at which the NPV becomes zero. It is necessary to iterate until this value is determined. The IRR is often used in assessing which projects should be pursued and even to rank projects. It may also be used to compare a project's return to other market returns. In general, the following rule should be followed in assessing whether a project should be pursued:

$$
\begin{aligned}
& I R R>W A C C, \text { accept the investment } \\
& I R R<W A C C, \text { reject the investment }
\end{aligned}
$$

If a project's investment is accepted, then it should be compared to other accepted projects. The higher the IRR, the better the investment. 


\section{Appendix E \\ LCOE}

The Levelized Cost of Electricity represents the annual costs leveraged over the lifetime and the electricity generated during the lifetime. It is used to compare the production costs of different energy technologies. To calculate the LCOE, the following steps should be followed:

1. Discount the annual cash outflows to their present value.

2. Discount the annual electricity generation to its present value.

3. Sum the present values of annual cash outflows to determine the net discounted cash outflow.

4. Sum the present values of annual electricity generation to determine the net discounted electricity generation.

5. Divide the net discounted cash outflow by the net discounted electricity generation.

Higher costs or cash outflows result in lower profits. As a result, the lower the LCOE, the better the energy technology is economically. Other factors may influence the LCOE, such as government regulations. For example, government subsidies or carbon taxes may lower the LCOE for non- or low-emitting technologies. 


\section{Appendix F \\ Excel SMR Model Inputs}

Table F1: Microsoft Excel Model Inputs

Reactor Information

SMR Rated Power (MWe)

180

Number of SMRs on site

Rated Power Large Reactor (MWe)

1260

Specific Overnight Capital Large Reactor $(\$ / K W e)$

3767

SMR Capacity Factor

0.9

Large Reactor Capacity Factor

0.9

Proportion of direct costs

0.66

Proportion of indivisible costs

0.34

Detailed Design and Engineering Costs (\$)

500000000

Technology Learning Rate (to NOAK)

0.045

Annual Operational Specific Costs

O\&M Specific Costs (\$/MWh)

18

Fuel + Decommissioning Specific Costs (\$/MWh)

9.1

Economic Data

Interest Rate/Cost of Debt

$10.00 \%$

Cost of Equity

$10.00 \%$

Share of Debt

$50.00 \%$

Share of Equity

$50.00 \%$

Weighted Average Cost of Capital (WACC)

$10.00 \%$

Inflation Rate

$2.50 \%$

Corporate Tax Rate

$35.00 \%$

Real Discount Rate

$7.32 \%$

Nominal Discount Rate

$9.82 \%$

Market Data

Electricity Price $(\$ / \mathrm{kWh})$

Construction Information

Number of Years Construction SMR

Interest Rate

$7.32 \%$

Number of Years large reactor 


\section{Appendix G \\ Excel SMR Model Outputs}

The FOAK and NOAK TCIC results for various plant configurations are calculated in the Excel file. Table G1 displays the results for the mid-range FOAK SMRs. Table G2 displays the effects of learning on the cost per unit at each site, assuming each site has identical total rated power. Table G3 displays the mid-range annual flows for NOAK SMRs, which are used to calculate the NPV, IRR, and LCOE for the specified inputs in Appendix F.

Table G1: Mid-Range TCIC Calculations for Various Plant Configurations for FOAK SMRs

\begin{tabular}{|c|c|c|c|c|c|c|c|c|}
\hline & \multicolumn{4}{|c|}{ FOAK } & \multicolumn{4}{|c|}{ FOAK } \\
\hline & Single & Twin Unit (2) & Two Twin Units (4) & Multi-Module (7) & Single & Twin Unit (2) & $\begin{array}{c}\text { Two Twin Units } \\
\text { (4) }\end{array}$ & $\begin{array}{l}\text { Multi-Module } \\
\text { (7) }\end{array}$ \\
\hline SMR Rated Electric Power (MWe) & \multicolumn{4}{|c|}{180} & \multicolumn{4}{|c|}{180} \\
\hline Modular Design Factor & \multicolumn{4}{|c|}{0.77} & \multicolumn{4}{|c|}{-} \\
\hline Co-siting Factor & \multicolumn{4}{|c|}{0.71} & \multicolumn{4}{|c|}{-} \\
\hline LR Rated Power (MWe) & \multicolumn{4}{|c|}{1260} & \multicolumn{4}{|c|}{ - } \\
\hline LR Total Cost (\$ billion) & \multicolumn{4}{|c|}{$\$ 4.75$} & \multicolumn{4}{|c|}{-} \\
\hline LR Overnight Specific Capital Cost (\$/kWe) & \multicolumn{4}{|c|}{$\$ 3,767.00$} & \multicolumn{4}{|c|}{-} \\
\hline Scaling Factor $\mathrm{n}(0.45-0.7)$ & \multicolumn{4}{|c|}{0.51} & \multicolumn{4}{|c|}{-} \\
\hline Scaled Capital Cost (\$ billion) & \multicolumn{4}{|c|}{$\$ 1.76$} & \multicolumn{4}{|c|}{ - } \\
\hline Scaled Overnight Specific Capital (\$/kWe) & \multicolumn{4}{|c|}{$\$ 9,774.48$} & \multicolumn{4}{|c|}{-} \\
\hline $\begin{array}{c}\text { Sharing of Systems One Pair Twin Units } \\
\text { Learning }(74-85 \%)\end{array}$ & - & 0.80 & \multicolumn{2}{|c|}{0.80} & - & 0.80 & \multicolumn{2}{|c|}{0.80} \\
\hline $\begin{array}{c}\text { Sharing of Systems Two Pair Twin Units } \\
\text { Learning }(82-95 \%)\end{array}$ & - & - & \multicolumn{2}{|c|}{0.89} & - & - & \multicolumn{2}{|c|}{0.89} \\
\hline Factory Fabrication Learning (0-2\%) & - & - & \multicolumn{2}{|c|}{0.01} & - & - & \multicolumn{2}{|c|}{0.01} \\
\hline Per unit cost factor FOAK & 1.35 & - & - & - & - & - & - & - \\
\hline Availability/Capacity Factor & \multicolumn{4}{|c|}{0.90} & \multicolumn{4}{|c|}{-} \\
\hline Per unit cost factor one pair twin units & - & 0.90 & - & - & - & 0.90 & - & - \\
\hline Per unit cost factor 2 pairs twin units & - & - & 0.87 & - & - & - & 0.87 & - \\
\hline Per unit cost factor 5-6 units multi-module & - & - & - & 0.75 & - & - & - & 0.75 \\
\hline Contingency Cost Increase & \multicolumn{4}{|c|}{1.05} & \multicolumn{4}{|c|}{-} \\
\hline SMR Specific Capital Cost (\$/kWe) & $\$ 8,282.29$ & $\$ 5,506.19$ & $\$ 5,316.24$ & $\$ 4,625.20$ & $\$ 5,535.22$ & $\$ 4,967.86$ & $\$ 4,796.49$ & $\$ 4,173.00$ \\
\hline SMR Capital Cost (\$ million) & $\$ 1,490.81$ & $\$ 991.11$ & $\$ 956.92$ & $\$ 832.54$ & $\$ 996.34$ & $\$ 894.22$ & $\$ 863.37$ & $\$ 751.14$ \\
\hline Total Factor Increase from LR & 2.20 & 1.46 & 1.41 & 1.23 & 1.58 & 1.32 & 1.27 & 1.11 \\
\hline DD\&E Costs (\$/kWe) & $\$ 62.50$ & $\$ 62.50$ & $\$ 62.50$ & $\$ 62.50$ & - & - & - & - \\
\hline SMR Total Specific Capital Cost (\$/kWe) & $\$ 628.19$ & $\$ 417.63$ & $\$ 403.22$ & $\$ 350.81$ & $\$ 419.83$ & $\$ 376.80$ & $\$ 363.80$ & $\$ 316.51$ \\
\hline SMR Total Capital Cost per Unit (\$million) & $\$ 8,972.98$ & $\$ 5,986.32$ & $\$ 5,781.97$ & $\$ 5,038.51$ & $\$ 5,955.06$ & $\$ 5,344.66$ & $\$ 5,160.29$ & $\$ 4,489.52$ \\
\hline SMR Total Plant Capital Cost (\$ million) & $\$ 1,615.14$ & $\$ 1,077.54$ & $\$ 1,040.75$ & $\$ 906.93$ & $\$ 1,071.91$ & $\$ 962.04$ & $\$ 928.85$ & $\$ 808.11$ \\
\hline
\end{tabular}


Table G2: 10 Year Sample of Annual Cash Flows for Mid-Range Single-Unit NOAK SMRs

\begin{tabular}{|c|c|c|c|c|c|c|c|c|c|c|}
\hline Year & Construction & O\&M & Fuel & $\begin{array}{c}\text { Power } \\
\text { Production } \\
\text { (MWe) } \\
\end{array}$ & Income & Net Cash Flows & $\begin{array}{c}\text { Cumulative Cash } \\
\text { Flows } \\
\end{array}$ & $\begin{array}{c}\text { Total Cash } \\
\text { Outflows/Costs }\end{array}$ & $\begin{array}{c}\text { Discounted Cash } \\
\text { Flows } \\
\end{array}$ & $\begin{array}{c}\text { Discounted Energy } \\
\text { kWh } \\
\end{array}$ \\
\hline 0 & $(290,199,105.96)$ & - & - & - & - & $(290,199,105.96)$ & $(290,199,105.96)$ & $(290,199,105.96)$ & $(290,199,105.96)$ & 0.00 \\
\hline 1 & $(290,199,105.96)$ & - & - & - & - & $(290,199,105.96)$ & $(580,398,211.92)$ & $(290,199,105.96)$ & $(270,412,803.28)$ & 0.00 \\
\hline 2 & $(290,199,105.96)$ & - & - & - & - & $(290,199,105.96)$ & $(870,597,317.89)$ & $(290,199,105.96)$ & $(251,975,566.70)$ & 0.00 \\
\hline 3 & $(290,199,105.96)$ & $(28,382,400.00)$ & $(14,348,880.00)$ & 162 & $139,073,760.00$ & $(193,856,625.96)$ & $(1,064,453,943.85)$ & $(332,930,385.96)$ & $(269,368,603.62)$ & $1,148,187,095.23$ \\
\hline 4 & $(290,199,105.96)$ & $(28,382,400.00)$ & $(14,348,880.00)$ & 162 & $139,073,760.00$ & $(193,856,625.96)$ & $(1,258,310,569.81)$ & $(332,930,385.96)$ & $(251,002,562.47)$ & $1,069,901,611.46$ \\
\hline 5 & $(290,199,105.96)$ & $(28,382,400.00)$ & $(14,348,880.00)$ & 162 & $139,073,760.00$ & $(193,856,625.96)$ & $(1,452,167,195.77)$ & $(332,930,385.96)$ & $(233,888,751.39)$ & $996,953,774.32$ \\
\hline 6 & $(290,199,105.96)$ & $(56,764,800.00)$ & $(28,697,760.00)$ & 324 & $278,147,520.00$ & $(97,514,145.96)$ & $(1,549,681,341.73)$ & $(375,661,665.96)$ & $(245,914,400.63)$ & $1,857,959,306.68$ \\
\hline 7 & $(290,199,105.96)$ & $(56,764,800.00)$ & $(28,697,760.00)$ & 324 & $278,147,520.00$ & $(97,514,145.96)$ & $(1,647,195,487.70)$ & $(375,661,665.96)$ & $(229,147,509.68)$ & $1,731,280,263.05$ \\
\hline 8 & $(290,199,105.96)$ & $(56,764,800.00)$ & $(28,697,760.00)$ & 324 & $278,147,520.00$ & $(97,514,145.96)$ & $(1,744,709,633.66)$ & $(375,661,665.96)$ & $(213,523,815.83)$ & $1,613,238,426.93$ \\
\hline 9 & $(290,199,105.96)$ & $(85,147,200.00)$ & $(43,046,640.00)$ & 486 & $417,221,280.00$ & $(1,171,665.96)$ & $(1,745,881,299.62)$ & $(418,392,945.96)$ & $(221,597,560.92)$ & $2,254,867,346.73$ \\
\hline
\end{tabular}




\section{Appendix $\mathrm{H}$ \\ Alaska Case Study}

There are regions of the United States where electricity rates are unusually high, such as Hawaii and parts of Alaska. In many of these regions, populations are small, making large power plants too costly. Remote rural areas in Alaska must rely solely on diesel electricity production, as natural gas is unavailable, and their electricity prices vary drastically. In 2010 , prices ranged from $\$ 0.50-\$ 1.50 / \mathrm{kWh}$ [67]. Rural Alaska incorporates 187 remote villages that may only be accessed via air and water only [68]. An SMR may be an ideal solution to the problem. It could be tailored to a very close supply and demand match, have long refueling cycles, and even account for future electricity needs. Additionally, due to the weather conditions in these regions, ships carrying the entire winter supply of diesel, gas, heating oil, etcetera must be brought in during a short summer period. If the window is missed, then it must be flown in, or shipped in via a tanker and perhaps even an icebreaker, adding up to an additional $\$ 0.50 / \mathrm{kWh}[69]$.

Alaska Center for Energy and Power assessed several towns that may require enough load for SMRs to be an effective solution [70]. One major problem is that most SMRs operate beyond $10 \mathrm{MWe}$ and the required load for these towns is a fraction of that. The following are examples of villages and their required average annual electricity load: Bethel-4.5 MWe, Dillingham-2.3 MWe, Galena-1 MWe, Kotzebue-2.4 MWe, Naknek-2.2 MWe, Nome-3.3 MWe, and Unalaska-3.8 MWe [70].

To make an investment feasible the annual specific capital costs must be less than their current electricity prices. For Bethel, for example, the average price from nuclear energy would have to be less than $\$ 1 / \mathrm{kWh}$. If it is assumed that a $5 \mathrm{MWe}$ Light Water SMR previously tested, proven, and fully mass manufactured could be produced and installed and operate with a capacity factor near $100 \%$. For such small electricity production, the total capital is relatively low at $\$ 178-\$ 240$ million, but the specific capital is much higher at $\$ 35,678-\$ 48.072 / \mathrm{kWe}$. Table I1 shows an estimate including the single NOAK SMR used to calculate these numbers.

Table I1: Bethel, Alaska 5 MWe Unit Capital Costs

\begin{tabular}{|c|c|c|c|c|c|c|}
\hline & \multicolumn{3}{|c|}{ FOAK Site } & \multicolumn{3}{|c|}{ NOAK Site } \\
\hline Co-Siting Factor & \multicolumn{3}{|c|}{1.00} & \multicolumn{3}{|c|}{ - } \\
\hline Modular Design Factor & \multicolumn{3}{|c|}{0.59} & \multicolumn{3}{|c|}{-} \\
\hline Learning Rate up to NOAK & \multicolumn{3}{|c|}{-} & \multicolumn{3}{|c|}{$4.50 \%$} \\
\hline & Low & Mid & High & Low & Mid & High \\
\hline Specific Capital Costs (\$/kWe) & $\$ 47,523$ & $\$ 55,777$ & $\$ 64,031$ & $\$ 35,678$ & $\$ 41,875$ & $\$ 48,072$ \\
\hline $\begin{array}{l}\text { Total Capital Costs } \\
\text { (\$ millions) }\end{array}$ & $\$ 238$ & $\$ 279$ & $\$ 320$ & $\$ 178$ & $\$ 209$ & $\$ 240$ \\
\hline
\end{tabular}

Table I 2 shows the results obtained for IRR, LCOE, and NPV using the model values for O\&M and fuel costs and over a range of electricity prices up to $\$ 1 / \mathrm{kWh}$. The capital costs used in this analysis are for the $5 \mathrm{MWe}$ SMR and were split over the three years of construction. However, the required average output for Bethel is only 4.5 MWe. Thus, this value was used for the rated power, O\&M, and fuel costs, and it reflects a $90 \%$ capacity factor. The LCOE for the project is calculated as $\$ 383-\$ 517 / \mathrm{MWh}$.

Table I2: Bethel, Alaska IRR, LCOE, and NPV for a NOAK 5 MWe SMR Site

\begin{tabular}{|c|c|c|c|}
\hline $\begin{array}{c}\text { Assumed Electricity } \\
\text { Price/kWh }\end{array}$ & NPV (\$ millions) & IRR & LCOE \\
\hline $60 c / \mathrm{kWh}$ & $(\$ 36)-\$ 102$ & $8.5-11.3 \%$ & \multirow{2}{*}{$\$ 383-\$ 517 / \mathrm{MWh}$} \\
\hline $80 \mathrm{c} / \mathrm{kWh}$ & $\$ 56-\$ 197$ & $11.3-14.7 \%$ & \multirow{2}{*}{} \\
\hline
\end{tabular}


Because the development and mass production of 5 MWe SMRs may not be feasible, analyzing the costs associated with the FOAK unit may prove more accurate. From Table I1, the specific FOAK capital cost including DD\&E costs is $\$ 47,523-\$ 64,031 / \mathrm{kWe}$ with a total capital of $\$ 238-\$ 320$ million. With the same assumptions as the single NOAK SMR case, the LCOE is then calculated as $\$ 511-\$ 678 / \mathrm{MWh}$. For this project to be feasible, the electricity price must be $82 \phi / \mathrm{kWh}$, resulting in an IRR of $8.9-11.7 \%$ and NPV of \$65-\$142 million. Either a FOAK or NOAK unit would ultimately reduce electricity costs for the region. Additionally, any excess power provided by this reactor could feasibly be used for industrial processes or district heating.

Other more heavily populated regions in Alaska have more reasonable demand loads which may warrant installation of SMR plants with multiple units. Additionally, it is much more likely that these types of plants would be mass produced in the future. Anchorage, for example, with an average annual demand load of 652 MWe may be suited for a scenario in which 6-100 MWe Light Water SMRs are installed [70]. This would nearly meet the entire required load. Table I3 shows the capital costs associated with both FOAK and NOAK units, demonstrating drastically reduced specific capital costs.

Table I3: Anchorage, Alaska 100 MWe SMR Capital Costs

\begin{tabular}{|c|c|c|c|c|c|c|}
\hline & \multicolumn{3}{|c|}{ FOAK Site* } & \multicolumn{3}{|c|}{ NOAK Site } \\
\hline Co-Siting Factor & \multicolumn{3}{|c|}{0.72} & \multicolumn{3}{|c|}{-} \\
\hline Modular Design Factor & \multicolumn{3}{|c|}{0.69} & \multicolumn{3}{|c|}{-} \\
\hline Learning Rate up to NOAK & \multicolumn{3}{|c|}{-} & \multicolumn{3}{|c|}{$4.50 \%$} \\
\hline Specific Capital Costs (\$/kWe) & Low & Mid & High & Low & Mid & High \\
\hline Single Unit & $\$ 9,294$ & $\$ 10,899$ & $\$ 12,504$ & $\$ 5,718$ & $\$ 6,706$ & $\$ 7,694$ \\
\hline Multi-Module & $\$ 5,859$ & $\$ 6,114$ & $\$ 6,374$ & $\$ 4,129$ & $\$ 5,056$ & $\$ 6,049$ \\
\hline $\begin{array}{l}\text { Total Capital Costs Per Unit (\$ } \\
\text { millions) }\end{array}$ & Low & Mid & High & Low & Mid & High \\
\hline Single Unit & $\$ 929$ & $\$ 1,090$ & $\$ 1,250$ & $\$ 572$ & $\$ 671$ & $\$ 769$ \\
\hline Multi-Module Unit & $\$ 586$ & $\$ 611$ & $\$ 637$ & $\$ 413$ & $\$ 506$ & $\$ 605$ \\
\hline
\end{tabular}

According to Anchorage Municipal Light and Power, the current residential electricity prices due energy costs are $\$ 0.10-\$ 0.11 / \mathrm{kWh}$ with total rate charges up to $\$ 0.14 / \mathrm{kWh}$ [71]. The FOAK IRR for this electricity cost of $\$ 0.11 / \mathrm{kWh}$ is less than $10 \%$, which is not high enough for the project to be profitable. As a result, FOAK units are not feasible in the area due to competition with less expensive generating technologies. Table I4 shows the NPV, LCOE, and IRR for a NOAK 6-unit plant, demonstrating that a NOAK plant may be competitive in this market for low and mid-range capital costs.

Table I4: Anchorage, Alaska IRR and NPV for a NOAK 6-100 MWe SMR Site

\begin{tabular}{|c|c|c|c|}
\hline $\begin{array}{c}\text { Assumed Electricity } \\
\text { Price/kWh }\end{array}$ & NPV (\$ millions) & IRR & LCOE \\
\hline 11 c/kWh & $\$ 524-\$ 1,314$ & $9.4-13.4 \%$ & $\$ 72-\$ 92 / \mathrm{MWh}$ \\
\hline
\end{tabular}




\section{Appendix I \\ Electricity Markets, Locational Based Marginal Pricing and Merit Order Usage}

Electricity markets all function differently and are governed by regulatory bodies, such as the New York Independent System Operator (ISO), which are responsible for matching the capacity available with the actual electricity consumer demand or load demand. In the NY ISO market, Power

Suppliers/Generators (Gen) offer to sell their energy, and Load Serving Entities (LSE) make bids on these offers. Gens are the facilities supplying energy, while LSEs are the entities that provide/sell the energy to customers (NY ISO 2014c). The NY ISO oversees these purchases and then determines the Locational Based Marginal Pricing (LBMP), as in the figure below. The NY ISO utilizes "a methodology where the price of energy at each location in the NYS Transmission System is equivalent to the cost to supply the next increment of load at that location," taking both the Gen offer prices and the actual transmission system into consideration (NY ISO 2014c). This will include the effects of losses and potential congestion.

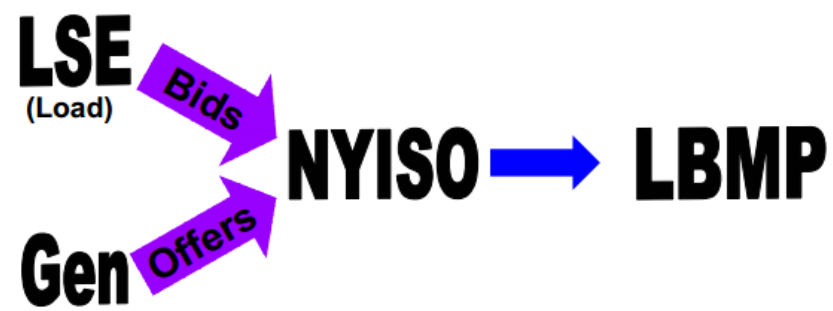

The overall process of determining the LBMP or marginal pricing is as follows:

1. "Determine the least costly way of producing electric energy to meet the load.

2. The price of producting an additional MW in a specific location in the grid is called the Locational Based Marginal Price (LBMP).

3. The LBMP will be the same at all buses when:

- Transmission limits are not reached

- Transmission limits are not exceeded

- Losses are zero" (NY ISO 2014c)

As part of determining the "least costly way of producing electric energy to meet the load," a merit order has been established (NY ISO 2014c). This helps establish the order in which Gen purchases are made by the LSE based on the operational costs for energy generating technologies. The following table gives an example of some variable operating costs for several technologies arranged in the order in which they would be deployed. The production costs include O\&M and fuel costs and are also shown in the table. This means that if wind energy is available it would all be purchased; then the nuclear, coal, natural gas, and finally petroleum energies would be used.

\begin{tabular}{|l|l|l|}
\hline Technology & Variable Operating Costs & Production Costs \\
\hline Wind & $\$ 0 / \mathrm{MWh}$ & \$10/MWh (IRENA 2012b) \\
\hline Nuclear & $\$ 7.5 / \mathrm{MWh}$ & $\$ 24 / \mathrm{MWh}(\mathrm{NEI} 2012)$ \\
\hline Coal & $\$ 25.5 / \mathrm{MWh}$ & $\$ 32.7 / \mathrm{MWh}(\mathrm{NEI} 2012)$ \\
\hline Natural Gas & $\$ 29.3 / \mathrm{MWh}$ & $\$ 34 / \mathrm{MWh}(\mathrm{NEI} 2012)$ \\
\hline Petroleum & $\$ 203.7 / \mathrm{MWh}$ & $\$ 224.8 / \mathrm{MWh}(\mathrm{NEI} 2012)$ \\
\hline
\end{tabular}




\section{Appendix J \\ Excel NHES Model Inputs and General Methodology}

Table H1: Excel NHES Model Inputs

Prices

Hydrogen $(\$ / \mathrm{kg})$

8

Efficiency

Wind

$30.00 \%$

Nuclear

$90.00 \%$

CAES

$70.00 \%$

Pumped Hydro

$90.00 \%$

Hydrogen

$65.00 \%$

\section{Capacity (MW)}

Wind

418.9

Nuclear

1639.5

Coal

0

Natural Gas

775

Storage

600

Operational Costs (\$/MWh)

Wind

Nuclear

27.1

Natural Gas

34

Storage

0.57

Coal

32.7

Hydrogen $(\$ / \mathrm{kg})$

1.74

\section{Conversion Factor}

Hydrogen (MWh/kg)

0.0329

The NHES Model calculates the operational costs for 2013 for both electric-only generation and electric generation combined with hydrogen or heat sales. Tables I2 and I3 display some values from the Excel spreadsheet in order to detail the methodology of the calculations. 
Table I2: 12 Hours of NHES Model Spreadsheet

\begin{tabular}{|c|c|c|c|c|c|c|c|c|c|c|}
\hline Hour & $\begin{array}{c}\text { Actual Load } \\
\text { (MWe) }\end{array}$ & Nuclear & Wind & $\begin{array}{c}\text { Additional } \\
\text { Capacity }\end{array}$ & $\begin{array}{c}\text { Available } \\
\text { for } \\
\text { Storage }\end{array}$ & $\begin{array}{c}\text { Actually } \\
\text { Stored }\end{array}$ & $\begin{array}{c}\text { Storage } \\
\text { Available to } \\
\text { Grid }\end{array}$ & $\begin{array}{c}\text { Peak } \\
\text { Demand } \\
\text { from Storage }\end{array}$ & $\begin{array}{c}\text { Natural } \\
\text { Gas } \\
\text { needed? }\end{array}$ & $\begin{array}{c}\text { Natural Gas } \\
\text { neded }\end{array}$ \\
\hline 0 & 1610.5 & 1639.5 & 125.67 & -154.67 & 154.67 & 154.67 & 108.269 & 0 & 0 & 0 \\
\hline 1 & 1541.1 & 1639.5 & 125.67 & -224.07 & 224.07 & 224.07 & 156.849 & 0 & 0 & 0 \\
\hline 2 & 1493.6 & 1639.5 & 125.67 & -271.57 & 271.57 & 271.57 & 190.099 & 0 & 0 & 0 \\
\hline 3 & 1474.2 & 1639.5 & 125.67 & -290.97 & 290.97 & 290.97 & 203.679 & 0 & 0 & 0 \\
\hline 4 & 1489 & 1639.5 & 125.67 & -276.17 & 276.17 & 276.17 & 193.319 & 0 & 0 & 0 \\
\hline 5 & 1551.3 & 1639.5 & 125.67 & -213.87 & 213.87 & 213.87 & 149.709 & 0 & 0 & 0 \\
\hline 6 & 1693.2 & 1639.5 & 125.67 & -71.97 & 71.97 & 71.97 & 50.379 & 0 & 0 & 0 \\
\hline 7 & 1878.3 & 1639.5 & 125.67 & 113.13 & 0 & 0 & 0 & 0 & 113.13 & 113.13 \\
\hline 8 & 1976.3 & 1639.5 & 125.67 & 211.13 & 0 & 0 & 0 & 0 & 211.13 & 211.13 \\
\hline 9 & 2042.8 & 1639.5 & 125.67 & 277.63 & 0 & 0 & 0 & 401.23 & 0 & 0 \\
\hline 10 & 2083.4 & 1639.5 & 125.67 & 318.23 & 0 & 0 & 0 & 380.83 & 0 & 0 \\
\hline 11 & 2121.5 & 1639.5 & 125.67 & 356.33 & 0 & 0 & 0 & 393.63 & 0 & 0 \\
\hline
\end{tabular}

In this system, the base-load nuclear power is always generating at its maximum capacity. Wind energy is generating at $30 \%$ efficiency and is assumed to have constant output. At this point, it necessary to determine whether additional natural gas is necessary to meet the demand or whether excess energy needs to be stored. This is performed by calculation the additional capacity required and then using IF statements to determine how much is actually stored and then available to the grid during peak demand:

1. Additional Capacity $=$ Actual Load - Nuclear - Wind

2. $\operatorname{IF}(G 3<0, A B S(G 3), 0)$

If the Additional Capacity is negative, then storage is needed and the absolute value of the Additional Capacity is available for storage. Otherwise, $0 \mathrm{MWe}$ is available for storage.

3. $\operatorname{IF}(\operatorname{IF}(G 3<0, A B S(G 3), 0)>\operatorname{Inputs!} \$ B \$ 19$, Inputs! $\$ B \$ 19, \operatorname{IF}(G 3<0, A B S(G 3), 0))$

If the Available for Storage value is greater than the maximum storage value or $600 \mathrm{MWe}$, then $600 \mathrm{MWe}$ is stored. Otherwise, the Available for Storage value is stored.

4. The storage efficiency is applied, determining the Storage Available to the Grid.

5. $\operatorname{IF}(\operatorname{SUM}(K 10: K 21)>H 22, H 22, \operatorname{SUM}(K 10: K 21))$

If the Storage Available to the Grid from the previous 12 hours is greater than the Additional Capacity required (non-zero values only), then the Additional Capacity Required is used. Otherwise, the Storage Available over the previous 12 hours is used. 
6. $\quad I F(H 3>L 3, H 3-L 3,0)$

If the non-zero Additional Capacity value is greater than the Peak Demand from Storage, then the difference represents the Natural Gas needed. Otherwise, zero natural gas is required.

7. $I F(M 3>$ Inputs! $\$ B \$ 18$, Inputs! $\$ B \$ 18, M 3)$

If the Natural Gas Needed value is greater than the maximum natural gas output, then the max output value is provided. Otherwise, the Natural Gas Needed value is produced.

In this particular scenario, the maximum natural gas capacity is not met. For this reason, details on determining the reserve equations and if statements are not included in this Appendix. The process is similar to what was done above.

Table I3: 12 Hours of NHES Model Spreadsheet to Demonstrate Hydrogen Sales

\begin{tabular}{|c|c|c|c|c|}
\hline Hour & $\begin{array}{c}\text { Actual Load } \\
\text { (MWe) }\end{array}$ & $\begin{array}{c}\text { Total } \\
\text { Electricity To } \\
\text { Grid }\end{array}$ & $\begin{array}{c}\text { Heat Energy } \\
\text { Available for } \\
\text { Hydrogen (MWth-hr) }\end{array}$ & $\begin{array}{c}\text { Kilograms of } \\
\text { Hydrogen } \\
\text { Produced }\end{array}$ \\
\hline 0 & 1610.5 & 1610.5 & 328.09 & 9972.28 \\
\hline 1 & 1541.1 & 1541.1 & 475.30 & 14446.81 \\
\hline 2 & 1493.6 & 1493.6 & 576.06 & 17509.35 \\
\hline 3 & 1474.2 & 1474.2 & 617.21 & 18760.15 \\
\hline 4 & 1489 & 1489 & 585.82 & 17805.93 \\
\hline 5 & 1551.3 & 1551.3 & 453.66 & 13789.17 \\
\hline 6 & 1693.2 & 1693.2 & 152.66 & 4640.23 \\
\hline 7 & 1878.3 & 1878.3 & 0.00 & 0.00 \\
\hline 8 & 1976.3 & 1976.3 & 0.00 & 0.00 \\
\hline 9 & 2042.8 & 2166.4 & 0.00 & 0.00 \\
\hline 10 & 2083.4 & 2146 & 0.00 & 0.00 \\
\hline 11 & 2121.5 & 2158.8 & 0.00 & 0.00 \\
\hline
\end{tabular}

To determine the hydrogen production and sales, the following methods were used:

1. $\quad I F\left((K 3-L 3)>0, \frac{K 3-L 3}{0.33}, 0\right)$

If Storage Available to Grid minus the Peak Demand Required from Storage is positive, then the heat energy available for hydrogen production is that value divided by the thermal-electric conversion efficiency. Otherwise, the value is zero.

2. Kilograms of Hydrogen Produced is calculated by dividing the Heat Energy Available for Hydrogen by the Conversion factor shown in Table 10 . 